\title{
Lepton asymmetry from mixing and oscillations
}

\author{
A. Kartavtsev, ${ }^{a}$ P. Millington ${ }^{b, c}$ and H. Vogel ${ }^{a}$ \\ ${ }^{a}$ Max-Planck-Institut für Physik, \\ Föhringer Ring 6, 80805 München, Germany \\ ${ }^{b}$ Physik Department T\%0, Technische Universität München, \\ James-Franck-Straße, 85748 Garching, Germany \\ ${ }^{c}$ School of Physics and Astronomy, University of Nottingham, \\ Nottingham NG7 2RD, United Kingdom \\ E-mail: alexander.kartavtsev@mpp.mpg.de, \\ p.millington@nottingham.ac.uk, hvogel@mpp.mpg.de
}

ABSTRaCt: We show how the two physically-distinct sources of $C P$-asymmetry relevant to scenarios of leptogenesis: (i) resonant mixing and (ii) oscillations between different flavours can be unambiguously identified within the Kadanoff-Baym formalism. These contributions are isolated by analyzing the spectral structure of the non-equilibrium propagators without relying on the definition of particle number densities. The mixing source is associated with the usual mass shells, whereas the oscillation source is identified with a third intermediate shell. In addition, we identify terms lying on the oscillation shell that can be interpreted as the destructive interference between mixing and oscillation. We confirm that identical shell structure is obtained in both the Heisenberg- and interaction-picture realizations of the Kadanoff-Baym formalism. In so doing, we illustrate the self-consistency and complementarity of these two approaches. The interaction-picture approach in particular has the advantage that it may be used to analyze all forms of mass spectra from quasi-degenerate through to hierarchical.

KEYworDs: Thermal Field Theory, Effective field theories

ARXIV EPRINT: 1601.03086 


\section{Contents}

1 Introduction 1

2 Shell structure for two-particle mixing in the Heisenberg picture 4

3 Shell structure for two-particle mixing in the interaction picture $\quad 11$

4 Comparison with the density matrix approximation $\quad 24$

5 Comparison with the effective Yukawa approach $\quad 26$

$\begin{array}{llr}6 & \text { Phenomenological implications } & 29\end{array}$

$\begin{array}{lll}7 & \text { Conclusions and outlook } & 35\end{array}$

$\begin{array}{ll}\text { A Non-equilibrium field theory } & 38\end{array}$

B Discrete symmetry transformations $\quad 45$

$\begin{array}{lll}\text { C Rate equations in the radiation-dominated universe } & 48\end{array}$

\section{Introduction}

In the presence of $C P$ violation, particle mixing and oscillations can provide two physicallydistinct sources of $C P$-asymmetry. In the quark sector, mixing arises due to the misalignment of the weak and Yukawa eigenbases, which gives rise to the CKM matrix of the Standard Model, whose complex entries provide the $C P$ violation observed in the $K, B$ and $B_{s}$ systems [1]. Oscillations, on the other hand, arise due to the formation of coherences in populations of particles with the same quantum numbers. These coherences are of particular interest in medium, leading for instance to oscillations via regeneration, as occurs for the $K_{0}-\bar{K}_{0}$ system in the presence of nuclear matter [2]. A similar distinction between mixing and oscillations can be identified in the cascade decays of heavy particles [3-6]. In extensions of the Standard Model, the physical relevance of these two sources of $C P$-asymmetry has also been identified in the context of leptogenesis (see e.g. refs. [711]), where, in certain scenarios, it is acknowledged that both effects must be accounted for in order to obtain quantitatively accurate predictions of the baryon asymmetry of the universe.

Leptogenesis [12] (for an overview, see e.g. ref. [13]) provides a potential explanation for the observed baryon asymmetry of the universe. It relies upon the existence of heavy righthanded Majorana neutrinos, whose out-of-equilibrium decays in the early universe are able to produce a net lepton number. This lepton asymmetry is subsequently converted to the 
observed baryon asymmetry through the sphaleron processes of the standard electroweak theory [14]. Whereas it is widely accepted that the source of $C P$-asymmetry provided by the mixing of different heavy-neutrino flavours is important for all mass spectra, the relative importance of the source provided by coherent oscillations between populations of heavyneutrino flavours is still under debate. Even so, one would anticipate that oscillations are most relevant when the mass spectrum of the heavy neutrinos is quasi-degenerate, where it has long been recognized that flavour effects play a significant role both from the heavy-neutrino [15-24] and charged-lepton sectors [25-33]. Thus, one would expect flavour oscillations to provide a significant source of $C P$-asymmetry in scenarios of resonant leptogenesis $[15,34,35]$. In such models, heavy-neutrino self-energy effects dominate [36$39]$ and provide a resonant enhancement of the leptonic $C P$-asymmetry, when the mass difference of at least two of the heavy neutrinos is comparable to their decay widths [15, 34]. In this context, it has recently been observed that the mixing and oscillation sources of lepton asymmetry can be of equal magnitude and the same sign in the strong-washout regime [9-11] (for a summary, see ref. [40]). This leads to a factor of two enhancement in the final lepton asymmetry, when both sources, rather than only one, are included, thereby expanding the viable parameter space for successful leptogenesis. However, it remains an open question as to what extent these two distinct phenomena and the interplay between them are captured by competing approaches.

In order to determine the asymmetry generated in scenarios of leptogenesis, it is necessary to solve systems of transport equations, akin to the classical Boltzmann equations (see e.g. refs. [41, 42]), that describe the time evolution of particle number densities. The impact of flavour oscillations can be accounted for through the quantum improvement of the classical Boltzmann equations by promoting the number densities of individual flavours to a matrix of densities [43], thereby allowing for flavour coherences. This approach yields the so-called density matrix formalism, which has been applied extensively to scenarios of leptogenesis $[9,10,21,24,28,44-49]$. On the other hand, a semi-classical treatment of mixing is possible through the inclusion of effective Yukawa couplings [15, 35], which can account for the $\varepsilon$ - and $\varepsilon^{\prime}$-type $C P$ violation, arising respectively from self-energy and vertex effects. Recently, there has been much progress in the literature [7, 8, 11, 50-72, 72-74] aiming to go beyond these semi-classical treatments and obtain 'first-principles' field-theoretic analogues of the Boltzmann equation. Often, these quantum transport equations are derived by means of the Kadanoff-Baym (KB) formalism [75, 76] (for reviews, see refs. [77, 78]), itself embedded in the Schwinger-Keldysh $[79,80]$ closed-time path formalism (see also refs. [8183]) of non-equilibrium thermal field theory. These approaches have the advantage that all quantum-mechanical effects are in principle accounted for consistently and systematically. However, an outstanding difficulty of such approaches is in the approximations needed to make the solution tractable and to extract physically-meaningful observables. As a result, it is often not straightforward to compare directly the results of different analyses or to ascertain to what extent relevant physical effects are accounted for.

In this article, we illustrate how the mixing and oscillation sources of lepton asymmetry can be identified unambiguously in the Kadanoff-Baym formalism by means of the spectral structure of the resummed heavy-neutrino propagators and independently of the definition 
of particle number densities. In the context of a toy two-flavour model, we will show that this spectral structure contains three distinct shells: two of these shells correspond to mixing and can be associated with the quasi-particle mass shells, whereas the third, which can be identified with oscillations, lies at an intermediate energy. In addition, we identify terms lying on the oscillation shell that can be interpreted as the interference between oscillation and mixing. In so doing, we provide a further illustration of the interplay of these two effects in the generation of lepton asymmetry. Most significantly, we find that this interference is destructive. With respect to the "benchmark" of the Boltzmann approximation (effective Yukawa couplings but flavour-diagonal number densities), this destructive interference can be viewed as a suppression of the oscillation source. Conversely, with respect to the "benchmark" of the density matrix approximation (tree-level Yukawa couplings but flavour-off-diagonal number densities), this destructive interference can be viewed as a suppression of the mixing source. This observation may in part account for apparent discrepancies between competing approaches and is anticipated to be of relevance to scenarios of resonant leptogenesis. Nevertheless, in spite of this destructive interference and in the weak-washout regime, we find that the oscillation and mixing sources can be of equal magnitude and contribute additively to the final asymmetry in agreement with the conclusions of refs. [9-11].

Aside from illustrating the interplay of these sources of $C P$-asymmetry, we perform the calculations in two very different approaches, namely the Heisenberg- and interactionpicture realizations of non-equilibrium quantum field theory. In contrast to earlier approaches, the interaction-picture description introduced in ref. [84] (see ref. [85] for a summary) enables one to proceed in a perturbative loop-wise fashion without encountering so-called pinch singularities [86-92] or secular terms [78] thought previously to spoil such approaches to non-equilibrium field theory. Quite remarkably, we find exact agreement between these two formulations, illustrating the self-consistency and complementarity of these two approaches. Working in the interaction picture has the particular advantage that all forms of mass spectra can be analyzed using a single method, from quasi-degenerate through to hierarchical.

In order to reduce the technical complications to a minimum and yet to include all qualitatively important effects for the generation of the asymmetry, we consider a simple toy model studied previously in refs. [57, 58, 61, 72, 93, 94]. The model contains one complex $(b)$ and two real scalar fields $\left(\psi_{i}\right)$ :

$$
\mathcal{L}=\frac{1}{2} \partial^{\mu} \psi_{i} \partial_{\mu} \psi_{i}-\frac{1}{2} \psi_{i} M_{i j}^{2} \psi_{j}+\partial^{\mu} \bar{b} \partial_{\mu} b-m^{2} \bar{b} b-\frac{\lambda}{2 ! 2 !}(\bar{b} b)^{2}-\frac{h_{i}}{2 !} \psi_{i} b b-\frac{h_{i}^{*}}{2 !} \psi_{i} \bar{b} \bar{b},
$$

where $\bar{b}$ denotes the Hermitian conjugate of $b$. Here and in the following, we assume summation over repeated indices, unless otherwise specified. Despite its simplicity, the model incorporates all features relevant for leptogenesis. The real scalar fields $\psi_{i}$ imitate the (two lightest) heavy right-handed neutrinos, whereas the complex scalar field $b$ models the leptons. The U(1) symmetry, which we use to define "lepton" number, is explicitly broken by the presence of the last two terms, just as the $B-L$ symmetry is explicitly broken by Majorana mass terms in phenomenological models. Thus, the first Sakharov condition [95] 
is fulfilled. The couplings $h_{i}$ model the complex Yukawa couplings of the right-handed neutrinos to the charged-leptons and the Higgs doublet. By rephasing the complex scalar field, at least one of the couplings $h_{i}$ can be made real. If $\arg \left(h_{1}\right) \neq \arg \left(h_{2}\right)$, the other one remains complex and there is $C$-violation, as is required by the second Sakharov condition.

The remainder of this article is organized as follows. Using the Heisenberg-picture realization of the Kadanoff-Baym formalism, we confirm in section 2 that the mixing and oscillation between different flavours indeed provide two distinct sources of lepton asymmetry. In section 3, we repeat the analysis in the interaction picture, finding identical results. Subsequently, in section 4, we make comparison with the density matrix approach and, in section 5, describe the inclusion of mixing effects via effective Yukawa couplings. Finally, in section 6 , we discuss the phenomenological implications of these two sources of lepton asymmetry, as well as their interference, and present numerical results. Our conclusions are presented in section 7 . In appendix A, we provide a brief outline of the details of the Kadanoff-Baym formalism pertinent to the analysis of this article. In addition, we summarize our definitions and notational conventions, making comparison with those that appear in the literature. In appendix B, we discuss the transformation properties of the model under generalized discrete symmetries and emphasize the need to specify $C$-symmetric initial conditions in the weak-washout regime. A derivation of the rate equations in an expanding universe, relevant to the study of leptogenesis in the strong-washout regime, is presented in appendix $\mathrm{C}$.

\section{Shell structure for two-particle mixing in the Heisenberg picture}

In this section, we show that the mixing and oscillation between different flavours provide two distinct sources of lepton asymmetry, in agreement with arguments presented in refs. [9-11]. Whereas the standard mixing contributions [34, 35] are associated with the mass shells $\omega_{i}(i=1,2)$ of the corresponding quasi-particles, the oscillation contribution is associated with an intermediate shell at $\bar{\omega}=\left(\omega_{1}+\omega_{2}\right) / 2$, which we will refer to as the oscillation shell in the remainder of this paper. In order to identify this structure, we first analyze the generation of the lepton asymmetry using the Kadanoff-Baym equations in the Heisenberg picture, as were previously applied to the toy model from eq. (1.1) in ref. [72].

Asymmetry in the absence of washout. Following refs. [66, 72], we assume that the complex scalar field forms a thermal bath with temperature $T$ and neglect the backreaction. The system begins its evolution at $t=-\infty$ in an equilibrium state. At $t=0$, the real scalars are brought out of equilibrium by an external source, thereby fulfilling the third Sakharov condition. This leads to the production of an asymmetry between the number densities of $b$ and $\bar{b}$. As time goes by, this asymmetry is erased by washout processes. Finally, at $t=\infty$, the system again reaches thermal equilibrium.

The expression for the produced asymmetry can be derived by considering the volume integral of the conserved Noether current:

$$
J_{\mu}(x)=\left\langle j_{\mu}(x)\right\rangle=i \lim _{y \rightarrow x}\left[\partial_{\mu_{x}} S_{<}(x, y)-\partial_{\mu_{y}} S_{>}(x, y)\right]
$$


where $S_{>}(x, y) \equiv\langle b(x) \bar{b}(y)\rangle$ and $S_{<}(x, y) \equiv\langle\bar{b}(y) b(x)\rangle$ are the Wightman propagators of the complex scalar field. Using the Kadanoff-Baym equations for $S_{\gtrless}$, which are similar to those that we will discuss below for the real scalar fields (see eq. (2.3)), and taking the equal-time limit $x_{0}=y_{0}=t$, we obtain the kinetic equation for the produced asymmetry, which takes into account quantum corrections to both the source and washout terms. Details of the derivation, together with the discussion of the approximations used, can be found in ref. [72].

Washout processes are physically very important and must be taken into account in a phenomenological analysis. On the other hand, in the analysis limited to the source term alone, one can neglect them, as was previously done in refs. [66, 72]. In this approximation, the produced asymmetry is given by

$$
\begin{aligned}
\eta(t) \equiv \int \mathrm{d}^{3} \mathbf{x}\left\langle j_{0}(t, \mathbf{x})\right\rangle= & -2 \operatorname{Im} H_{12} \int_{-\infty}^{t} \mathrm{~d} x^{0} \int_{-\infty}^{t} \mathrm{~d} y^{0} \int_{\mathbf{q}} \\
& \times i\left[G_{<}^{12}\left(x^{0}, y^{0}, \mathbf{q}\right) \Pi_{>}\left(y^{0}, x^{0}, \mathbf{q}\right)-G_{>}^{12}\left(x^{0}, y^{0}, \mathbf{q}\right) \Pi_{<}\left(y^{0}, x^{0}, \mathbf{q}\right)\right],
\end{aligned}
$$

where $H_{i j} \equiv h_{i} h_{j}^{*}$, the functions $G_{\gtrless}^{12}$ are components of the Wightman propagators of the real scalar fields $\psi_{i}$, and $\Pi_{\gtrless}$ are the self-energies with the couplings $\left(h_{i}\right)$ "amputated". We use the shorthand notation $\int_{\mathbf{q}} \equiv \int \frac{\mathrm{d}^{3} \mathbf{q}}{(2 \pi)^{3}}$. A comprehensive summary of the various propagators and self-energies, their definitions and useful identities, as well as the differing nomenclature used throughout the literature is provided in appendix A. The expression for the asymmetry in eq. (2.2) is entirely equivalent to the one obtained via the definition of particle number densities used in the interaction-picture approach to the Kadanoff-Baym formalism, developed in ref. [84]. In section 6 , we present numerical solutions of eq. (2.2) for $C$-symmetric initial conditions.

Solution of the Kadanoff-Baym equations. The Wightman propagators in eq. (2.2) are solutions to the Kadanoff-Baym equations for the mixing fields $\psi_{i}$. In the absence of external sources, these transport equations read [58]

$$
\left[\square_{x} \delta_{i k}+M_{i k}^{2}\right] G_{\gtrless}^{k j}(x, y)=\int_{-\infty}^{y^{0}} \mathrm{~d}^{4} z \Pi_{\gtrless}^{i k}(x, z) G_{\rho}^{k j}(z, y)-\int_{-\infty}^{x^{0}} \mathrm{~d}^{4} z \Pi_{\rho}^{i k}(x, z) G_{\gtrless}^{k j}(z, y),
$$

where $M_{i j}$ are the mass parameters of the renormalized Lagrangian, $G_{\rho}^{i j}$ is the spectral function, and $\Pi_{\gtrless}^{i j}$ and $\Pi_{\rho}^{i j}$ are the Wightman and spectral self-energies, respectively. Using the definitions of the retarded and advanced propagators, and the self-energies in appendix A, we can rewrite eq. (2.3) in a form more convenient for the analysis that follows:

$$
\left[\square_{x} \delta_{i k}+M_{i k}^{2}\right] G_{\gtrless}^{k j}(x, y)=-\int_{z}\left[\Pi_{R}^{i k}(x, z) G_{\gtrless}^{k j}(z, y)+\Pi_{\gtrless}^{i k}(x, z) G_{A}^{k j}(z, y)\right] .
$$

We have also made use of the fact that

$$
\int_{-\infty}^{x_{0}} \mathrm{~d} z_{0} \int \mathrm{d}^{3} \mathbf{z}=\int_{-\infty}^{+\infty} \mathrm{d} z_{0} \int \mathrm{d}^{3} \mathbf{z} \theta\left(x_{0}-z_{0}\right) \equiv \int_{z} \theta\left(x_{0}-z_{0}\right) .
$$


The Kadanoff-Baym equations for the retarded and advanced propagators can be derived from eq. (2.3):

$$
\left[\square_{x} \delta_{i k}+M_{i k}^{2}\right] G_{R(A)}^{k j}(x, y)=-\int_{z} \Pi_{R(A)}^{i k}(x, z) G_{R(A)}^{k j}(z, y)+\delta^{4}(x-y) \delta^{i j} .
$$

For our purposes, it is sufficient to know that, at the one-loop level to which we limit ourselves here, the self-energies of the real scalar fields are translationally invariant in the thermal bath. This implies, in particular, that eq. (2.6) admits a translationally-invariant solution. Using eq. (2.6), one can readily check that

$$
G_{\gtrless}^{i j}(x, y)=-\int_{u, v} G_{R}^{i m}(x, u) \Pi_{\gtrless}^{m n}(u, v) G_{A}^{n j}(v, y)
$$

is a solution to eq. (2.4). Since, as is discussed above, the self-energies, as well as the retarded and advanced propagators on the r.h.s. of eq. (2.7) are translationally invariant, the 1.h.s. of eq. (2.7) is also translationally invariant. In other words, eq. (2.7) is an equilibrium solution for the Wightman propagators.

In the setup considered here, the system is assumed to be brought out of equilibrium instantaneously by an external source at $t=0$. While it is hard to imagine a physicallymotivated scenario that would generate such an initial condition, this assumption will allow us to solve the equations analytically and access qualitative features of the solution important also for phenomenologically-viable initial conditions. The source can be considered as a bi-local contribution to the self-energy. Following refs. [66, 72], we consider an external source that leaves the spectral function unperturbed. Thus, both of the Wightman self-energies are "perturbed" in the same way, $\Pi_{\gtrless}^{m n}(u, v) \rightarrow \Pi_{\gtrless}^{m n}(u, v)-K^{m n}(u, v)$, with $K^{m n}(u, v)=\delta\left(u^{0}\right) \delta\left(v^{0}\right) \mathcal{K}^{m n}(\mathbf{u}-\mathbf{v})$. The translational invariance of the one-loop self-energies in the thermal bath renders the Kadanoff-Baym equations linear, i.e. a sum of two solutions is also a solution. Using this linearity, we obtain the following equation for the non-equilibrium part $G_{\delta \gtrless}^{k j} \subset G_{\gtrless}^{k j}$ of the Wightman propagators induced by the external source:

$$
\left[\square_{x} \delta_{i k}+M_{i k}^{2}\right] G_{\delta \gtrless}^{k j}(x, y)=-\int_{z}\left[\Pi_{R}^{i k}(x, z) G_{\delta \gtrless}^{k j}(z, y)-K^{i k}(x, z) G_{A}^{k j}(z, y)\right] .
$$

Using eq. (2.6), one can readily check that $[66,72]$

$$
G_{\delta \gtrless}^{i j}(x, y)=\int_{u, v} G_{R}^{i m}(x, u) K^{m n}(u, v) G_{A}^{n j}(v, y)
$$

is a solution to eq. (2.8). In the absence of spacetime expansion, we are only interested in the non-equilibrium part of the resummed Wightman propagators, which are common to both the positive- and negative-frequency components, $G_{\delta>}^{i j}=G_{\delta<}^{i j} \equiv G_{\delta}^{i j}$. The sum of the equilibrium and non-equilibrium parts (eqs. (2.7) and (2.9)) gives the full solution of the Kadanoff-Baym equations in the thermal bath, as was studied in detail in ref. [72]. 
Shell structure of the non-equilibrium solution. The equilibrium solution in eq. (2.7) does not contribute to the asymmetry in agreement with the third Sakharov condition (see ref. [72] for more details) and will not be considered further. In order to unravel the shell structure of the non-equilibrium solution in eq. (2.9), we perform a Wigner transform (see appendix A). Using the relation between the double-momentum and Wigner representations (see eq. (A.9)) and neglecting sub-leading off-shell contributions (see e.g. section 6 of ref. [72]), we obtain

$$
G_{\delta}^{i j}\left(t, q_{0}>0\right) \approx \int_{0}^{\infty} \frac{\mathrm{d} p_{0}}{2 \pi} \int_{0}^{\infty} \frac{\mathrm{d} p_{0}^{\prime}}{2 \pi} 2 \pi \delta\left(q_{0}-\frac{1}{2}\left(p_{0}+p_{0}^{\prime}\right)\right) e^{-i\left(p_{0}-p_{0}^{\prime}\right) t} G_{R}^{i m}\left(p_{0}\right) \mathcal{K}^{m n} G_{A}^{n j}\left(p_{0}^{\prime}\right) .
$$

We will later analytically continue the real variable $q_{0}$ to the complex plane in order to apply Cauchy's theorem. The notation $q_{0}>0$ is therefore understood throughout this article to mean $\operatorname{Re} q_{0}>0$. In addition, we omit all dependence on the common three-momentum $\mathbf{q}$ when no ambiguity results. The explicit forms of the Wigner transforms of the retarded and advanced propagators can be inferred from eq. (2.6) using translational-invariance of the self-energies:

$$
G_{R(A)}^{i j}\left(q_{0}\right)=-\frac{\operatorname{adj} D_{R(A)}^{i j}\left(q_{0}\right)}{\operatorname{det} D_{R(A)}\left(q_{0}\right)}
$$

where

$$
D_{R(A)}^{i j}\left(q_{0}\right) \equiv q^{2} \delta^{i j}-M_{i j}^{2}-\Pi_{R(A)}^{i j}\left(q_{0}\right),
$$

and we use boldface for matrices in flavour space. Having not needed to employ the gradient expansion (cf. refs. [92, 96]), the leading self-energy corrections to the spectral structure of the non-equilibrium part of the propagator, specifically the shifts of the poles in the real and imaginary directions, have been taken into account.

The imaginary parts of the retarded (advanced) self-energies are odd under $q_{0} \rightarrow-q_{0}$, i.e. $\operatorname{Im} \Pi_{R(A)}^{i j}\left(q_{0}\right)=-\operatorname{Im} \Pi_{R(A)}^{i j}\left(-q_{0}\right)$, such that all four poles of $G_{R(A)}^{i j}\left(q_{0}\right)$ lie in the lowerhalf complex plane. These four poles correspond to the zeros of $\operatorname{det} \boldsymbol{D}_{R}\left(q_{0}\right)$ and lie at $q_{0}=\Omega_{i}$ and $q_{0}=-\Omega_{i}^{*}$, where

$$
\Omega_{i}=\omega_{i}-\frac{i}{2} \Gamma_{i}
$$

The real and imaginary parts of $\Omega_{i}$ correspond to the in-medium frequency $\omega_{i}$ and width $\Gamma_{i}$, respectively. In the neighbourhood of the poles with $q_{0}>0$, we can approximate [72]

$$
\operatorname{det} \boldsymbol{D}_{R}\left(q_{0}>0\right) \approx\left(q_{0}^{2}-\Omega_{1}^{2}\right)\left(q_{0}^{2}-\Omega_{2}^{2}\right),
$$

where it is assumed that the self-energies are slowly varying functions of $q_{0}$ for $q_{0} \sim \Omega_{i}$. We would like to emphasize that eq. (2.14) is not only applicable, but actually becomes exact in the degenerate limit. The implications of this pole approximation for the effective regulator of the lepton asymmetry will be discussed later in the context of degeneracy symmetry limits (see e.g. ref. [9]). Instead, if we were interested in the poles with $q_{0}<0$, we could approximate

$$
\operatorname{det} \boldsymbol{D}_{R}\left(q_{0}<0\right) \approx\left(q_{0}^{2}-\Omega_{1}^{* 2}\right)\left(q_{0}^{2}-\Omega_{2}^{* 2}\right)
$$


Using Cauchy's theorem to evaluate the integral in eq. (2.10) approximately, we arrive at the advertised three-shell structure

$$
\begin{aligned}
G_{\delta}^{i j}\left(t, q_{0}>0\right) \approx \frac{1}{\left|\Delta \Omega^{2}\right|^{2}}[ & \sum_{k=1}^{2} 2 \pi \delta\left(q_{0}-\omega_{k}\right) e^{-\Gamma_{k} t} \frac{\operatorname{adj} D_{R}^{i m}\left(\omega_{k}\right)}{2 \omega_{k}} \mathcal{K}^{m n} \frac{\operatorname{adj} D_{A}^{n j}\left(\omega_{k}\right)}{2 \omega_{k}} \\
& -2 \pi \delta\left(q_{0}-\bar{\omega}\right) e^{-i\left(\omega_{1}-\omega_{2}\right) t} e^{-\bar{\Gamma} t} \frac{\operatorname{adj} D_{R}^{i m}\left(\omega_{1}\right)}{2 \omega_{1}} \mathcal{K}^{m n} \frac{\operatorname{adj} D_{A}^{n j}\left(\omega_{2}\right)}{2 \omega_{2}} \\
& \left.-2 \pi \delta\left(q_{0}-\bar{\omega}\right) e^{-i\left(\omega_{2}-\omega_{1}\right) t} e^{-\bar{\Gamma} t} \frac{\operatorname{adj} D_{R}^{i m}\left(\omega_{2}\right)}{2 \omega_{2}} \mathcal{K}^{m n} \frac{\operatorname{adj} D_{A}^{n j}\left(\omega_{1}\right)}{2 \omega_{1}}\right],
\end{aligned}
$$

where we have defined the average in-medium decay width

$$
\bar{\Gamma}=\frac{1}{2}\left(\Gamma_{1}+\Gamma_{2}\right),
$$

and introduced

$$
\Delta \Omega^{2} \equiv \Omega_{2}^{2}-\Omega_{1}^{2}
$$

In eq. (2.16), the three distinct shells are identified by the frequencies $q_{0}=\omega_{i}(i=1,2)$ and $q_{0}=\bar{\omega} \equiv \frac{1}{2}\left(\omega_{1}+\omega_{2}\right)$. The shells with frequencies $q_{0}=\omega_{i}$ lie at the two poles of the retarded propagator, which can be associated with quasi-particle degrees of freedom. As such, these terms correspond to the contribution from mixing. On the other hand, the additional intermediate shell with frequency $q_{0}=\bar{\omega}$ corresponds to the contribution from oscillations and, as we will see, the interference between mixing and oscillations. This three-shell structure matches that obtained in ref. [96], which makes use of a gradient expansion of the KB equations. Therein, the authors also find an additional fourth shell with frequency $q_{0}=\omega_{1}-\omega_{2}$ corresponding to particle-anti-particle coherences, which are not considered in the present analysis.

In order to gain a better understanding of the shell structure and to make comparisons with the existing literature, we will now consider the non-equilibrium part of the propagator (eq. (2.16)) to leading order in powers of the self-energies. Specifically, we will neglect terms higher than first-order in the self-energies in the products of adjugate matrices in eq. (2.16). With regards to the lepton asymmetry, we are only interested in the off-diagonal components of this non-equilibrium part of the propagator. In the mass eigenbasis, in which the remainder of this article is understood, these components read

$$
\begin{aligned}
& G_{\delta}^{i \not}\left(t, q_{0}>0\right) \approx 2 \pi \delta\left(q_{0}-\omega_{i}\right) \frac{1}{2 \omega_{i}} e^{-\Gamma_{i} t} \delta n^{i i}(0) \Pi_{A}^{i \not}\left(\omega_{i}\right) R_{i \not} \\
& -2 \pi \delta\left(q_{0}-\omega_{i}\right) \frac{1}{2 \omega_{i}} e^{-\Gamma_{i} t} \delta n^{\not l t}(0) \Pi_{R}^{i \not}\left(\omega_{i}\right) R_{i \hbar} \\
& +2 \pi \delta\left(q_{0}-\bar{\omega}\right) \frac{1}{\left(2 \omega_{i}\right)^{\frac{1}{2}}\left(2 \omega_{i}\right)^{\frac{1}{2}}} e^{-i\left(\omega_{i}-\omega_{i}\right) t} e^{-\bar{\Gamma} t}\left[\delta n^{i \hbar}(0) \Delta M_{i \hbar}^{2}\right. \\
& \left.-\delta n^{i i}(0) \Pi_{A}^{i t}\left(\omega_{i}\right)+\delta n^{\not t}(0) \Pi_{R}^{i t}\left(\omega_{i}\right)\right] R_{i \neq} .
\end{aligned}
$$


In eq. (2.19), $\Delta M_{i \neq}^{2} \equiv M_{i}^{2}-M_{i}^{2}$ is the mass splitting, and we have employed the notation used in ref. [11]:

$$
\not \equiv \begin{cases}2, & i=1 \\ 1, & i=2 .\end{cases}
$$

In addition, for later convenience, we have introduced the following notation for the initial deviation of "particle number densities" from equilibrium:

$$
\delta n^{i j}(0) \equiv \frac{\mathcal{K}^{i j}}{\left(2 \omega_{i}\right)^{\frac{1}{2}}\left(2 \omega_{j}\right)^{\frac{1}{2}}} .
$$

Note, however, that the identification of the mixing and oscillation shells in eq. (2.16) is independent of this definition. Finally,

$$
R_{i \downarrow} \equiv \frac{\Delta M_{i \not}^{2}}{\left(\Delta M_{i \downarrow}^{2}\right)^{2}+\left(\omega_{i} \Gamma_{i}-\omega_{i} \Gamma_{\ell}\right)^{2}}
$$

is the effective regulator.

The first and second lines of eq. (2.19) live on the mass shells and describe the standard mixing contributions to the asymmetry. On the other hand, the third and fourth lines describe the contribution from oscillations and the interference between mixing and oscillations. In section 3, we will make use of the interaction-picture approach in order to isolate the interference terms from the pure oscillation terms. We note that the regulator in eq. (2.22) cannot be applied naively in the doubly-degenerate limit $M_{2} \rightarrow M_{1}$ and $\Gamma_{2} \rightarrow \Gamma_{1}$ (for a comparison of various regulators in degeneracy symmetry limits, see e.g. refs. $[9,66]$ ). Nevertheless, the last two terms of eq. (2.19) have structure similar to those of the $i$-th and $\not$-th mass shell terms, respectively, but with opposite signs. Therefore, there is a partial cancellation of these contributions, an effect that becomes important in the maximallyresonant regime, where the interference between mixing and oscillations is anticipated to be of most relevance. This cancellation has been analyzed in detail in refs. [66, 72], where it was demonstrated that, in the degenerate limit $\omega_{2} \rightarrow \omega_{1}$ and $\Gamma_{2} \rightarrow \Gamma_{1}$, back-reaction of mixing on the oscillation ensures exact cancellation in agreement with the physical expectations.

Mixing and oscillation sources of CP asymmetry. It remains for us to study how each term in the non-equilibrium propagator (eq. (2.19)) contributes to the asymmetry by substituting it into the source term (eq. (2.2)). As identified earlier, in the absence of cosmological expansion, we are only interested in the non-equilibrium part of the resummed Wightman propagators for which $G_{\delta>}^{i j}=G_{\delta<}^{i j}=G_{\delta}^{i j}$. In this case, the expression for the produced asymmetry (eq. (2.2)) simplifies to

$$
\eta(t)=-2 \operatorname{Im} H_{12} \int_{0}^{t} \mathrm{~d} x^{0} \int_{0}^{t} \mathrm{~d} y^{0} \int_{\mathbf{q}} G_{\delta}^{12}\left(x^{0}, y^{0}, \mathbf{q}\right) \Pi_{\rho}\left(y^{0}, x^{0}, \mathbf{q}\right),
$$

where we have taken into account that the system is brought out of equilibrium at $t=0$ in the lower limits of the time integration. Next, we trade $x^{0}$ and $y^{0}$ for the central and 
relative coordinates $t \equiv \frac{1}{2}\left(x^{0}+y^{0}\right)$ and $R^{0} \equiv x^{0}-y^{0}$. In addition, we use the Markovian approximation

$$
\int_{-2 t}^{2 t} \mathrm{~d} R^{0} \sin \left(R^{0} q_{0}\right) \cos \left(R^{0} p_{0}\right)=0, \quad \int_{-2 t}^{2 t} \mathrm{~d} R^{0} \sin \left(R^{0} q_{0}\right) \sin \left(R^{0} p_{0}\right) \approx \pi \delta\left(q_{0}-p_{0}\right) .
$$

In this way, we may rewrite eq. (2.23) in the differential form

$$
\frac{\mathrm{d} \eta}{\mathrm{d} t}=4 \operatorname{Im} H_{12} \int_{q_{0}, \mathbf{q}} \theta\left(q_{0}\right) \operatorname{Im} G_{\delta}^{12}\left(t, q_{0}, \mathbf{q}\right) \widetilde{\Pi}_{\rho}\left(q_{0}, \mathbf{q}\right)
$$

where we have restored the common momentum $\mathbf{q}$,

$$
\widetilde{\Pi}_{\rho}\left(q_{0}, \mathbf{q}\right) \equiv-i \Pi_{\rho}\left(q_{0}, \mathbf{q}\right)=\frac{1}{8 \pi} L_{\rho}\left(q_{0}, \mathbf{q}\right),
$$

(see eq. (A.22) and appendix A) and, in the $\overline{\mathrm{MS}}$ scheme,

$$
L_{\rho}\left(q_{0}, \mathbf{q}\right)=1+\frac{2 T}{|\mathbf{q}|} \ln \left(\frac{1-e^{-\left(q_{0}+|\mathbf{q}|\right) / 2 T}}{1-e^{-\left(q_{0}-|\mathbf{q}|\right) / 2 T}}\right)
$$

(see ref. [72] for more details). Substituting the expression for $G_{\delta}^{12}$ from eq. (2.19) into eq. (2.25), we obtain the following expression for the time-derivative of the asymmetry:

$$
\begin{aligned}
\frac{\mathrm{d} \eta}{\mathrm{d} t} \approx & 2 \sum_{i} \int_{\mathbf{q}} \frac{M_{i}}{\omega_{i}} e^{-\Gamma_{i} t} \delta n^{i i}(0, \mathbf{q}) \Gamma_{i}^{\mathrm{med}}\left(\omega_{i}, \mathbf{q}\right) \epsilon_{i}^{\operatorname{med}}\left(\omega_{i}, \mathbf{q}\right) \\
+ & 2 \operatorname{Im} H_{12} \operatorname{Im} \int_{\mathbf{q}} \frac{1}{\left(\omega_{1} \omega_{2}\right)^{\frac{1}{2}}} e^{-i\left(\omega_{1}-\omega_{2}\right) t} e^{-\bar{\Gamma} t} \widetilde{\Pi}_{\rho}(\bar{\omega}, \mathbf{q})\left[\delta n^{12}(0, \mathbf{q}) \Delta M_{12}^{2}\right. \\
& \left.-\delta n^{11}(0, \mathbf{q}) \Pi_{A}^{12}\left(\omega_{2}, \mathbf{q}\right)+\delta n^{22}(0, \mathbf{q}) \Pi_{R}^{12}\left(\omega_{1}, \mathbf{q}\right)\right] R_{12} .
\end{aligned}
$$

The first line of eq. (2.28) originates from the mass shell terms of eq. (2.19) and describes the mixing source of lepton asymmetry. The second and third lines stem from the oscillationshell terms of eq. (2.19) and contain the oscillation source and the interference between mixing and oscillations, which will be isolated in section 3 .

Before concluding this section, we comment in more detail on the physical content of eq. (2.28). Firstly, the overall factor of two arises because, in the toy model (eq. (1.1)), each decay of the heavy real scalar violates "lepton" number by two units. Secondly, we note that the mixing contribution has the standard structure [34, 35]. The asymmetry produced per unit time and unit volume is proportional to the departure from equilibrium $\delta n^{i i}$, the in-medium decay probability $\Gamma_{i}^{\text {med }}\left(\omega_{i}, \mathbf{q}\right)=\Gamma_{i} L_{\rho}\left(\omega_{i}, \mathbf{q}\right)$ and the in-medium asymmetry produced in each decay

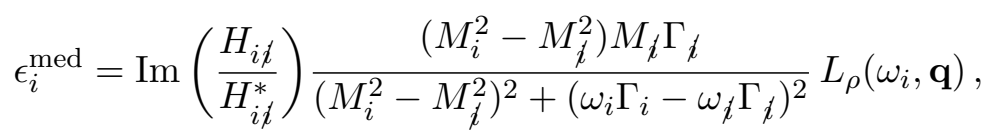

where the function $L_{\rho}\left(\omega_{i}, \mathbf{q}\right)$ takes into account quantum-statistical corrections to the decay width and $C$-violating parameter, respectively (see eq. $(2.26)$ and refs. [57, 58]). Thirdly, the leading contribution to the oscillation term is proportional to the off-diagonal element of the matrix of densities $\delta n^{12}$, as one might expect, sourcing asymmetry only in the presence of flavour coherences. 


\section{Shell structure for two-particle mixing in the interaction picture}

In this section, we show how the shell structure identified above in the Heisenberg picture is reproduced in the interaction picture.

Tree-level Wightman propagator. In the interaction-picture approach, the tree-level Wightman propagator can be obtained straightforwardly by evaluating the ensemble expectation value (EEV) of field operators directly (see appendix A and ref. [84]). In the double-momentum representation and assuming spatial homogeneity, it takes the form

$$
\begin{aligned}
G_{<}^{0, i j}\left(p, p^{\prime}, \tilde{t}\right)= & 2 \pi\left(2 \operatorname{sign}\left(p_{0}\right) p_{0}\right)^{1 / 2} \delta\left(p^{2}-M_{i}^{2}\right) e^{i\left(p_{0}-p_{0}^{\prime}\right) \tilde{t}} \\
& \times\left[\theta\left(p_{0}\right) \theta\left(p_{0}^{\prime}\right) n^{i j}(t, \mathbf{p})+\theta\left(-p_{0}\right) \theta\left(-p_{0}^{\prime}\right)\left(\delta^{i j}+n^{i j *}(t,-\mathbf{p})\right)\right](2 \pi)^{3} \delta^{3}\left(\mathbf{p}-\mathbf{p}^{\prime}\right) \\
& \times 2 \pi\left(2 \operatorname{sign}\left(p_{0}^{\prime}\right) p_{0}^{\prime}\right)^{1 / 2} \delta\left(p^{\prime 2}-M_{j}^{2}\right) .
\end{aligned}
$$

Since the system of interest is spatially isotropic, the number densities $n^{i j}(t, \mathbf{p})$ are functions only of $|\mathbf{p}|$, such that $n^{i j *}(t,-\mathbf{p})=n^{i j *}(t, \mathbf{p})$. Note that, in eq. (3.1), we have distinguished between a macroscopic time $t$ and a microscopic time $\tilde{t}$, as is necessary in the interaction picture (see appendix A). In the end, the physical limit will be obtained at equal times $X^{0}=\tilde{t}$ (for more details, see ref. [84]).

Dressed Wightman propagator. In order to find an explicit form for the dressed Wightman propagator, we restrict ourselves to the inclusion of one-loop self-energies and make use of the Markovian approximation. The latter has the effect of restoring exact energy-momentum conservation (see eq. (2.24)). Using in addition that the self-energies, and retarded and advanced propagators are translationally invariant (see section 2), the Schwinger-Dyson equation of the Wightman propagator reduces to

$$
\begin{aligned}
G_{<}^{i j}\left(p, p^{\prime}, \tilde{t}\right)= & G_{<}^{0, i j}\left(p, p^{\prime}, \tilde{t}\right)-G_{R}^{0, i k}(p) \Pi_{<}^{k l}(p)(2 \pi)^{4} \delta^{4}\left(p-p^{\prime}\right) G_{A}^{l j}\left(p^{\prime}\right) \\
& -G_{R}^{0, i k}(p) \Pi_{R}^{k l}(p) G_{<}^{l j}\left(p, p^{\prime}, \tilde{t}\right)-G_{<}^{0, i k}\left(p, p^{\prime}, \tilde{t}\right) \Pi_{A}^{k l}\left(p^{\prime}\right) G_{A}^{l j}\left(p^{\prime}\right),
\end{aligned}
$$

and that of the retarded (advanced) propagator to

$$
G_{R(A)}^{i j}(p)=G_{R(A)}^{0, i j}(p)-G_{R(A)}^{0, i k}(p) \Pi_{R(A)}^{k l}(p) G_{R(A)}^{l j}(p) .
$$

The result of the approximations described above is the elimination of convolution integrals over intermediate momenta in the Schwinger-Dyson equations. As was shown in ref. [11], this system may then be solved analytically for the resummed Wightman propagator, and we find

$$
G_{<}^{i j}\left(p, p^{\prime}, \tilde{t}\right)=F_{R}^{i k}(p) G_{<}^{0, k l}\left(p, p^{\prime}, \tilde{t}\right) F_{A}^{l j}\left(p^{\prime}\right)-G_{R}^{i k}(p) \Pi_{<}^{k l}(p)(2 \pi)^{4} \delta^{4}\left(p-p^{\prime}\right) G_{A}^{l j}\left(p^{\prime}\right),
$$

where we have defined

$$
\begin{aligned}
& F_{R}^{i j} \equiv \sum_{n=0}^{\infty}\left[\left(-G_{R}^{0} \cdot \Pi_{R}\right)^{n}\right]^{i j}=-G_{R}^{i k} D_{R}^{0, k j}=\delta^{i j}-G_{R}^{i k} \Pi_{R}^{k j}, \\
& F_{A}^{i j} \equiv \sum_{n=0}^{\infty}\left[\left(-\Pi_{A} \cdot G_{A}^{0}\right)^{n}\right]^{i j}=-D_{A}^{0, i k} G_{A}^{k j}=\delta^{i j}-\Pi_{A}^{i k} G_{A}^{k j} .
\end{aligned}
$$


The second term on the r.h.s. of eq. (3.4) describes equilibrium $\Delta L=0$ and $\Delta L=2$ scatterings. Instead, the part of interest to us is contained within the first term on the r.h.s. of eq. (3.4). In particular, we wish to study the part proportional to the deviation from equilibrium $\delta n^{i j}(t, \mathbf{p})$. Inserting the tree-level Wightman propagator from eq. (3.1) into eq. (3.4), this part is given by

$$
\begin{aligned}
\left.G_{\delta}^{i j}\left(p, p^{\prime}, \tilde{t}\right)\right|_{p_{0}, p_{0}^{\prime}>0}= & F_{R}^{i k}(p) 2 \pi\left(2 p_{0}\right)^{1 / 2} \delta_{+}\left(p^{2}-M_{k}^{2}\right) e^{i\left(p_{0}-p_{0}^{\prime}\right) \tilde{t}} \delta n^{k l}(t, \mathbf{p})(2 \pi)^{3} \delta^{3}\left(\mathbf{p}-\mathbf{p}^{\prime}\right) \\
& \times 2 \pi\left(2 p_{0}^{\prime}\right)^{1 / 2} \delta_{+}\left(p^{\prime 2}-M_{l}^{2}\right) F_{A}^{l j}\left(p^{\prime}\right)
\end{aligned}
$$

where

$$
2 \pi \delta_{+}\left(p^{2}-M_{i}^{2}\right) \equiv 2 \pi \theta\left(p_{0}\right) \delta\left(p^{2}-M_{i}^{2}\right)=\frac{1}{2 E_{i}}\left[\frac{i}{p_{0}-E_{i}+i \epsilon}-\frac{i}{p_{0}-E_{i}-i \epsilon}\right],
$$

and $E_{i}=\left(\mathbf{p}^{2}+M_{i}^{2}\right)^{\frac{1}{2}}$.

On-shell approximation. We will first illustrate that there is no contribution to the resummed non-equilibrium propagator in eq. (3.6) from the tree-level on-shell modes $p^{2}=M_{i}^{2}$. In so doing, we will also illustrate explicitly that eq. (3.6) is free of pinch singularities, which would potentially arise from ill-defined products of Dirac delta functions with identical arguments.

For this purpose, it is convenient to work with the Wigner transform (see appendix A) of the non-equilibrium part of the dressed Wightman propagator:

$$
\begin{aligned}
G_{\delta}^{i j}\left(q_{0}>0, X, \tilde{t}\right)= & \int_{Q_{0}} e^{-i Q_{0}\left(X^{0}-\tilde{t}\right)} F_{R}^{i k}\left(q_{0}+Q_{0} / 2\right) 2 \pi\left(2 E_{k}\right)^{1 / 2} \delta_{+}\left(\left(q_{0}+Q_{0} / 2\right)^{2}-E_{k}^{2}\right) \\
& \times \delta n^{k l}(t, \mathbf{q}) 2 \pi\left(2 E_{l}\right)^{1 / 2} \delta_{+}\left(\left(q_{0}-Q_{0} / 2\right)^{2}-E_{l}^{2}\right) F_{A}^{l j}\left(q_{0}-Q_{0} / 2\right)
\end{aligned}
$$

where the trivial $\mathbf{Q}$ integral has been performed. Hereafter, we omit three-momentum arguments for notational brevity. In order to perform the $Q_{0}$ integral, we will now assume erroneously that the only poles are those provided by the Dirac delta functions appearing explicitly in eq. (3.8). We emphasize that we should not anticipate that we will obtain the correct result, since $G_{R(A)}^{0}$ also contains poles.

By virtue of the properties of the Dirac delta function, we may show that

$$
\left(2 E_{i}\right)^{1 / 2} \delta\left(\left(q_{0} \pm Q_{0} / 2\right)^{2}-E_{i}^{2}\right)=2\left(2 E_{i}\right)^{-1 / 2} \sum_{s= \pm 1} \delta\left(Q_{0} \pm 2 q_{0} \mp 2 s E_{i}\right) .
$$

Performing the integral over $Q_{0}$, we then find

$$
G_{\delta}^{i j}\left(q_{0}>0, X, \tilde{t}\right)=e^{-i \Delta E_{k l}\left(X_{0}-\tilde{t}\right)} 2 \pi \delta\left(q_{0}-E_{k l}\right) F_{R}^{i k}\left(E_{k}\right) \frac{\delta n^{k l}(t, \mathbf{q})}{\left(2 E_{k}\right)^{1 / 2}\left(2 E_{l}\right)^{1 / 2}} F_{A}^{l j}\left(E_{l}\right),
$$

where $E_{k l} \equiv\left(E_{k}+E_{l}\right) / 2$. Equation (3.10) is well defined in a distributional sense and, in evaluating the tree-level poles, we have not encountered any singular behaviour, illustrating explicitly that the expression for the resummed propagator in eq. (3.6) is free of pinch singularities. 
One might be tempted to consider the terms in eq. (3.5) proportional to $G_{\mathrm{R}}^{i m} \Pi_{\mathrm{R}}^{m k}$ and $\Pi_{\mathrm{A}}^{l n} G_{\mathrm{A}}^{n j}$ as subleading. Were we to drop these contributions, we would find the following for the off-diagonal elements of eq. (3.10):

$$
G_{\delta}^{i t}\left(q_{0}>0, X, \tilde{t}\right)=e^{-i \Delta E_{i \tilde{f}}\left(X_{0}-\tilde{t}\right)} 2 \pi \delta\left(q_{0}-\bar{E}\right) \frac{\delta n^{i t}(t, \mathbf{q})}{\left(2 E_{i}\right)^{1 / 2}\left(2 E_{i}\right)^{1 / 2}} .
$$

Such an approximation for the resummed would-be heavy-neutrino propagator, when used in the equation for the asymmetry, discards the phenomenon of mixing, accounting only for oscillations between the two flavours, as identified in ref. [11]. In fact, as we will now show, the terms omitted in eq. (3.11) are of order unity, and $G_{\delta}^{i j}(q, X, \tilde{t})$ is identically zero due to the erroneous treatment of the pole structure in this on-shell approximation.

Considering the explicit form of the dressed retarded propagator in eq. (3.8), we may show that the factor

$$
F_{R}^{i k}\left(E_{k}\right)=\delta^{i k}-G_{R}^{i m}\left(E_{k}\right) \Pi_{R}^{m k}\left(E_{k}\right)=\delta^{i k}-\frac{\Delta M_{\not k k}^{2} \delta^{i m}+\left[\operatorname{adj} \Pi_{R}\left(E_{k}\right)\right]^{i m}}{\operatorname{det} \boldsymbol{D}_{R}\left(E_{k}\right)} \Pi_{R}^{m k}\left(E_{k}\right) .
$$

The determinant in the denominator of eq. (3.12) can be written as

$$
\operatorname{det} \boldsymbol{D}_{R}\left(E_{k}\right)=\Delta M_{k k}^{2} \Pi_{R}^{k k}\left(E_{k}\right)+\operatorname{det} \boldsymbol{\Pi}_{R}\left(E_{k}\right) .
$$

Thus, we have

$$
G_{R}^{i m}\left(E_{k}\right) \Pi_{R}^{m k}\left(E_{k}\right)=\frac{\Delta M_{l k}^{2} \Pi_{R}^{i k}\left(E_{k}\right)+\delta^{i k} \operatorname{det} \Pi_{R}\left(E_{k}\right)}{\Delta M_{\not k}^{2} \Pi_{R}^{k k}\left(E_{k}\right)+\operatorname{det} \Pi_{R}\left(E_{k}\right)},
$$

where we have also used the fact that

$$
\left[\operatorname{adj} \Pi_{R}\left(E_{k}\right)\right]^{i m} \Pi_{R}^{m k}\left(E_{k}\right)=\delta^{i k} \operatorname{det} \boldsymbol{\Pi}_{R}\left(E_{k}\right),
$$

by definition of the adjugate matrix. We may then show that

$$
G_{R}^{i m}\left(E_{k}\right) \Pi_{R}^{m k}\left(E_{k}\right)=\delta^{i k}, \quad \Pi_{\mathrm{A}}^{l n}\left(E_{l}\right) G_{\mathrm{A}}^{n j}\left(E_{l}\right)=\delta^{l j} .
$$

Substituting eq. (3.16) into the expression for the resummed propagator in eq. (3.10), it immediately follows that it is identically zero. Clearly, this result is incorrect. As we will now show, this is a consequence of having neglected the poles in $G_{R(A)}^{0}$, whose contributions are in fact pivotal in determining the correct form of the resummed propagator.

Pole structure. The tree-level retarded (advanced) propagator has the form

$$
G_{R(A)}^{0, i j}(p)=-\frac{\delta^{i j}}{p^{2}-M_{i}^{2} \pm i \epsilon \operatorname{sign}\left(p_{0}\right)}=-\mathcal{P} \frac{\delta^{i j}}{p^{2}-M_{i}^{2}} \pm i \pi \delta^{i j} \operatorname{sign}\left(p_{0}\right) \delta\left(p^{2}-M_{i}^{2}\right),
$$

where we have used the identity

$$
\frac{1}{x \pm i \epsilon}=\mathcal{P} \frac{1}{x} \mp i \pi \delta(x)
$$


in which $\mathcal{P}$ denotes the Cauchy principal value. Equation (3.18) may readily be confirmed by using the limit representations

$$
\delta(x)=\lim _{\epsilon \rightarrow 0^{+}} \frac{1}{\pi} \frac{\epsilon}{x^{2}+\epsilon^{2}}, \quad \mathcal{P} \frac{1}{x}=\lim _{\epsilon \rightarrow 0^{+}} \frac{x}{x^{2}+\epsilon^{2}} .
$$

Substituting eq. (3.17) into the non-equilibrium part of the resummed propagator (eq. (3.6)), it would appear that we have products of Dirac delta functions of identical arguments. However, we have seen already that eq. (3.6) is free of pinch singularities. The reason for this is that these pinch singularities are resummed, and it is by performing this resummation that we will obtain the correct form for the Wigner representation of the resummed Wightman propagator. In particular, both the equilibrium and non-equilibrium parts of the propagator acquire finite widths (cf. ref. [92]).

In order to understand the structure of this resummation, it is helpful to begin with the single-flavour case. Therein, we wish to evaluate the following structure:

$$
\begin{aligned}
I_{R} & \equiv \sum_{n=0}^{\infty}\left(-G_{\mathrm{R}}^{0} \cdot \Pi_{\mathrm{R}}\right)^{n} 2 \pi \operatorname{sign}\left(p_{0}\right) \delta\left(p^{2}-M^{2}\right) \\
& =\sum_{n=0}^{\infty}\left(\frac{\Pi_{R}}{p^{2}-M^{2}+i \epsilon \operatorname{sign}\left(p_{0}\right)}\right)^{n} 2 \pi \operatorname{sign}\left(p_{0}\right) \delta\left(p^{2}-M^{2}\right) .
\end{aligned}
$$

Note that we are free to insert the product $\operatorname{sign}\left(p_{0}\right) \operatorname{sign}\left(p_{0}^{\prime}\right)$ into the tree-level propagator eq. (3.1), since the signs of $p_{0}$ and $p_{0}^{\prime}$ are equal in the absence of particle-antiparticle correlations. We proceed by performing the following partial-fractioning, using the limit representation of the Dirac delta function in eq. (3.19):

$$
2 \pi \operatorname{sign}\left(p_{0}\right) \delta\left(p^{2}-M^{2}\right)=\frac{i}{p^{2}-M^{2}+i \epsilon \operatorname{sign}\left(p_{0}\right)}-\frac{i}{p^{2}-M^{2}-i \epsilon \operatorname{sign}\left(p_{0}\right)} .
$$

We then decompose

$$
I_{R} \equiv I_{R}^{+}-I_{R}^{-}
$$

where

$$
I_{R}^{ \pm}=i \sum_{n=0}^{\infty}\left(\frac{\Pi_{R}}{p^{2}-M^{2}+i \epsilon \operatorname{sign}\left(p_{0}\right)}\right)^{n} \frac{1}{p^{2}-M^{2} \pm i \epsilon \operatorname{sign}\left(p_{0}\right)} .
$$

By employing the distributional identity (see e.g. ref. [97])

$$
\left(\frac{1}{x \pm i \epsilon}\right)^{n}=\mathcal{P} \frac{1}{x^{n}} \mp \frac{(-1)^{n-1}}{(n-1) !} i \pi \delta^{(n-1)}(x),
$$

where $\delta^{(n)}(x)$ is the $n$-th derivative of the Dirac delta function, we find

$$
I_{R}^{+}=i \sum_{n=0}^{\infty} \mathcal{P}\left(\frac{1}{p^{2}-M^{2}}\right)^{n+1}\left(\Pi_{R}\right)^{n}+\sum_{n=0}^{\infty} \frac{\left(-\Pi_{R}\right)^{n}}{n !} \pi \operatorname{sign}\left(p_{0}\right) \delta^{(n)}\left(p^{2}-M^{2}\right)
$$

Transposing the identity in eq. (3.24), we may also show that

$$
\mathcal{P} \frac{1}{x^{n}}=\frac{(-1)^{n-1}}{(n-1) !} \mathcal{P}^{(n-1)} \frac{1}{x},
$$


where $\mathcal{P}^{(n)}(x)$ is the $n$-th derivative of the Cauchy principal value. Hence, we obtain

$$
I_{R}^{+}=i \sum_{n=0}^{\infty} \frac{\left(-\Pi_{R}\right)^{n}}{n !} \mathcal{P}^{(n)} \frac{1}{p^{2}-M^{2}}+\sum_{n=0}^{\infty} \frac{\left(-\Pi_{R}\right)^{n}}{n !} \pi \operatorname{sign}\left(p_{0}\right) \delta^{(n)}\left(p^{2}-M^{2}\right) .
$$

As we might expect from comparing $I_{R}^{+}$to the usual Feynman-Dyson series, this result is proportional to the resummed retarded propagator:

$$
I_{R}^{+}=i \sum_{n=0}^{\infty} \frac{\left(-\Pi_{R}\right)^{n}}{n !} \frac{\partial^{n}}{\partial\left(p^{2}\right)^{n}}\left(-G_{R}^{0}(p)\right)=-i G_{R}(p) .
$$

In the case of $I_{R}^{-}$, we instead have

$$
I_{R}^{-}=i \sum_{n=0}^{\infty}\left(\frac{\Pi_{R}}{p^{2}-M^{2}+i \epsilon \operatorname{sign}\left(p_{0}\right)}\right)^{n} \frac{1}{p^{2}-M^{2}-i \epsilon \operatorname{sign}\left(p_{0}\right)} .
$$

This term would appear to suffer from pinch singularities, arising from the product of poles at $p^{2}=M^{2}+i \epsilon \operatorname{sign}\left(p_{0}\right)$ and $p^{2}=M^{2}-i \epsilon \operatorname{sign}\left(p_{0}\right)$. However, such pinch singularities arise here only at a finite order in perturbation theory. This is a consequence of having artificially restored energy-momentum conservation through the Markovian approximation. It can be shown [84] that the perturbation series is in fact well defined so long as one takes into account finite-time effects and the microscopic violation of energy-momentum conservation. Performing the summation over $n$ in eq. (3.29) first, we can make use of the fact that

$$
\sum_{n=0}^{\infty}\left(\frac{\Pi_{R}}{p^{2}-M^{2}+i \epsilon \operatorname{sign}\left(p_{0}\right)}\right)^{n} \equiv \sum_{n=0}^{\infty}\left(\frac{\Pi_{R}}{p^{2}-M^{2}-i \epsilon \operatorname{sign}\left(p_{0}\right)}\right)^{n}=\frac{p^{2}-M^{2}}{p^{2}-M^{2}-\Pi_{R}}
$$

does not depend on the pole prescription when $\left|\operatorname{Im} \Pi_{R}\right|>\epsilon$. We may therefore write

$$
\begin{aligned}
I_{R}^{-} & \equiv i \sum_{n=0}^{\infty}\left(\frac{\Pi_{R}}{p^{2}-M^{2}-i \epsilon \operatorname{sign}\left(p_{0}\right)}\right)^{n} \frac{1}{p^{2}-M^{2}-i \epsilon \operatorname{sign}\left(p_{0}\right)} \\
& =i \sum_{n=0}^{\infty} \frac{\left(-\Pi_{R}\right)^{n}}{n !} \mathcal{P}^{(n)} \frac{1}{p^{2}-M^{2}}-\sum_{n=0}^{\infty} \frac{\left(-\Pi_{R}\right)^{n}}{n !} \pi \operatorname{sign}\left(p_{0}\right) \delta^{(n)}\left(p^{2}-M^{2}\right) .
\end{aligned}
$$

We see that $I_{R}^{-}$differs from $I_{R}^{+}$in eq. (3.27) by the sign of the second term. Hence, we arrive at the result

$$
I_{R}=\sum_{n=0}^{\infty} \frac{\left(-\Pi_{R}\right)^{n}}{n !} 2 \pi \operatorname{sign}\left(p_{0}\right) \delta^{(n)}\left(p^{2}-M^{2}\right)
$$

In order to understand the meaning of eq. (3.32) and why this contribution has not cancelled between $I_{R}^{+}$and $I_{R}^{-}$, we first write

$$
2 \pi \operatorname{sign}\left(p_{0}\right) \delta\left(p^{2}-M^{2}\right) \equiv \frac{i}{\left(p^{2}-M^{2}\right)^{+}}-\frac{i}{\left(p^{2}-M^{2}\right)^{-}},
$$


replacing the explicit $\epsilon$ by the following equivalent (but more general) prescription for deforming the contour in the complex plane. The + and - indicate that we are to deform the contour of integration in $p_{0}$ away from the real axis such that we pass always above $(+)$ or below $(-)$ the poles at $p^{2}-M^{2}=0$. Returning to the generalized Taylor series expansion in eq. (3.32), we see that it effects a shift $p^{2}-M^{2} \rightarrow p^{2}-M^{2}-\Pi_{R}$. Since this includes a shift of the poles in the imaginary direction, we must simultaneously deform the contour of integration such that no poles cross the contour during this shift. In this way, we have

$$
I_{R}=\frac{i}{\left(p^{2}-M^{2}-\Pi_{R}\right)^{+}}-\frac{i}{\left(p^{2}-M^{2}-\Pi_{R}\right)^{-}} .
$$

The r.h.s. of eq. (3.34) is the complex delta function (see e.g. refs. [98, 99]):

$$
I_{R}=2 \pi \delta\left(p^{2}-M^{2}-\Pi_{R}\right)
$$

which corresponds to the contribution from the poles of $i /\left(p^{2}-M^{2}-\Pi_{R}\right)$. Since the imaginary part of the retarded self-energy is odd under $p_{0} \rightarrow-p_{0}$, all of these poles lie in the lower-half complex plane. We can therefore write $I_{R}$ as

$$
I_{R}=\frac{i f\left(p_{0}\right)}{p^{2}-M^{2}-\Pi_{R}},
$$

where $f\left(p_{0}\right)$ is a single-valued and analytic function chosen such that we must close the contour of integration in the lower-half complex plane. Specifically, we require [98]: (i) $f\left(p_{0}\right) \approx 1$ in the vicinity of the poles, (ii) $f\left(p_{0}\right) \approx 0$, effectively, on the real axis far away from the poles, (iii) $f\left(p_{0}\right)$ regular near the real axis, and (iv) $f\left(p_{0}\right)$ vanishing far away in the lower-half complex plane.

We may proceed analogously in the case of two flavours:

$$
I_{R}^{i}=I_{R}^{i i}+I_{R}^{i \hbar} \equiv \sum_{n=0}^{\infty}\left[\left(-G_{R}^{0} \cdot \Pi_{R}\right)^{n}\right]^{i j} 2 \pi \operatorname{sign}\left(p_{0}\right) \delta\left(p^{2}-M_{j}^{2}\right) .
$$

We can make sense of the resummation in $I_{R}^{i i}$ by considering the series for the resummed propagator (no summation over $i$ implied):

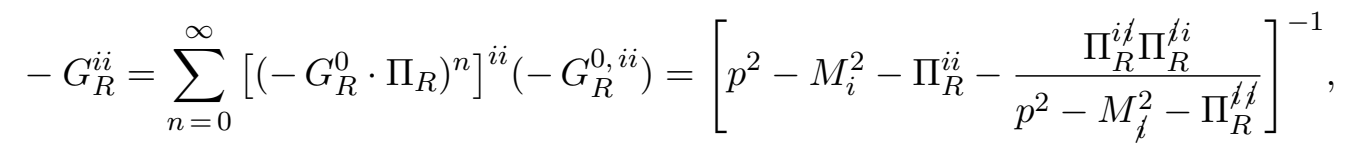

which differs from $I_{R}^{i i}$ by the replacement

$$
2 \pi \operatorname{sign}\left(p_{0}\right) \delta\left(p^{2}-M_{i}^{2}\right) \longrightarrow-G_{R}^{0, i i}(p) .
$$

In the case of $I_{R}^{i i}$, we can resum all but insertions of $1 /\left(p^{2}-M_{i}^{2}+i \epsilon \operatorname{sign}\left(p_{0}\right)\right)$ straightforwardly and obtain

$$
I_{\mathrm{R}}^{i i}=\sum_{n=0}^{\infty}\left[\frac{1}{p^{2}-M_{i}^{2}+i \epsilon \operatorname{sign}\left(p_{0}\right)}\left(\Pi_{R}^{i i}+\frac{\Pi_{R}^{i \not} \Pi_{R}^{\not i}}{p^{2}-M_{\not l}^{2}-\Pi_{R}^{\not k}}\right)\right]^{n} 2 \pi \operatorname{sign}\left(p_{0}\right) \delta\left(p^{2}-M_{i}^{2}\right),
$$


which gives eq. (3.38) on making the replacement in eq. (3.39). The potential pinch singularities in eq. (3.40) are resummed in the same way as for the single-flavour case, yielding

$$
\begin{aligned}
I_{\mathrm{R}}^{i i} & =\sum_{n=0}^{\infty} \frac{1}{n !}\left(-\Pi_{R}^{i i}-\frac{\Pi_{R}^{i \hbar} \Pi_{R}^{\not i}}{p^{2}-M_{\not}^{2}-\Pi_{R}^{k i}}\right)^{n} 2 \pi \operatorname{sign}\left(p_{0}\right) \delta^{(n)}\left(p^{2}-M_{i}^{2}\right) \\
& =2 \pi \delta\left(-\left[G_{R}^{-1}\right]^{i i}\right),
\end{aligned}
$$

where $\delta$ is understood to be the complex delta function, giving the contribution from the poles of

$$
i\left[p^{2}-M_{i}^{2}-\Pi_{R}^{i i}-\frac{\Pi_{R}^{i \ell} \Pi_{R}^{t i}}{p^{2}-M_{\not}^{2}-\Pi_{R}^{i l t}}\right]^{-1} .
$$

This is equal to the contribution from the poles of

$$
i\left[\operatorname{adj} \boldsymbol{D}_{R}\right]^{i i} / \operatorname{det} \boldsymbol{D}_{R}
$$

which occur at $\operatorname{det} \boldsymbol{D}_{R}=0$. Hence, we have

$$
I_{\mathrm{R}}^{i i}=2 \pi\left[\operatorname{adj} \boldsymbol{D}_{R}\right]^{i i} \delta\left(\operatorname{det} \boldsymbol{D}_{R}\right)
$$

For the series $I_{R}^{i t}$, we are able to resum all but insertions of $i /\left(p^{2}-M_{\ell}^{2}+i \epsilon \operatorname{sign}\left(p_{0}\right)\right)$ straightforwardly and obtain

$$
\begin{aligned}
& I_{R}^{i f}=\frac{\Pi_{R}^{i f}}{p^{2}-M_{i}^{2}-\Pi_{R}^{i i}} \\
& \times \sum_{n=0}^{\infty}\left[\frac{1}{p^{2}-M_{\not l}^{2}+i \epsilon \operatorname{sign}\left(p_{0}\right)}\left(\Pi_{R}^{\not t}+\frac{\Pi_{R}^{i i} \Pi_{R}^{i \downarrow}}{p^{2}-M_{i}^{2}-\Pi_{R}^{i i}}\right)\right]^{n} 2 \pi \operatorname{sign}\left(p_{0}\right) \delta\left(p^{2}-M_{\grave{l}}^{2}\right) \\
& =\frac{\Pi_{R}^{i \not}}{p^{2}-M_{i}^{2}-\Pi_{R}^{i i}} \sum_{n=0}^{\infty} \frac{1}{n !}\left(-\Pi_{R}^{\not \lambda}-\frac{\Pi_{R}^{i i} \Pi_{R}^{i t}}{p^{2}-M_{i}^{2}-\Pi_{R}^{i i}}\right)^{n} 2 \pi \operatorname{sign}\left(p_{0}\right) \delta^{(n)}\left(p^{2}-M_{\not}^{2}\right) \\
& =\frac{\Pi_{R}^{i t}}{p^{2}-M_{i}^{2}-\Pi_{R}^{i i}} 2 \pi \delta\left(-\left[G_{R}^{-1}\right]^{i \lambda}\right) .
\end{aligned}
$$

We may readily verify that this gives the resummed propagator $G_{R}^{i t}(p)$ on making the replacement

$$
2 \pi \operatorname{sign}\left(p_{0}\right) \delta\left(p^{2}-M_{\not}^{2}\right) \longrightarrow-G_{R}^{0, \not \lambda}(p)
$$

Equation (3.45) corresponds to the contribution from the poles of

$$
-i G_{R}^{i j}=\frac{i \Pi_{R}^{i j}}{\left(p^{2}-M_{i}^{2}-\Pi_{R}^{i i}\right)\left(p^{2}-M_{i}^{2}-\Pi_{R}^{i k}\right)-\Pi_{R}^{i j} \Pi_{R}^{i i}}=i\left[\operatorname{adj} \boldsymbol{D}_{R}\right]^{i j} / \operatorname{det} \boldsymbol{D}_{R},
$$

which again occur at $\operatorname{det} \boldsymbol{D}_{R}=0$. Hence, we have

$$
I_{\mathrm{R}}^{i \neq}=2 \pi\left[\operatorname{adj} \boldsymbol{D}_{R}\right]^{i t} \delta\left(\operatorname{det} \boldsymbol{D}_{R}\right) .
$$


Continuing similarly for the remaining components and the corresponding advanced series $\left(I_{A} \equiv I_{R}^{*}\right)$, we obtain the complete expression for the non-equilibrium part of the resummed propagator:

$$
\begin{aligned}
G_{\delta}^{i j}\left(p, p^{\prime}, \tilde{t}\right)= & 2 \pi\left(2 \operatorname{sign}\left(p_{0}\right) p_{0}\right)^{1 / 2}\left[\operatorname{adj} \boldsymbol{D}_{R}(p)\right]^{i k} \delta\left(\operatorname{det} \boldsymbol{D}_{R}(p)\right) e^{i\left(p_{0}-p_{0}^{\prime}\right) \tilde{t}} \\
& \times\left[\theta\left(p_{0}\right) \theta\left(p_{0}^{\prime}\right) \delta n^{k l}(t, \mathbf{p})+\theta\left(-p_{0}\right) \theta\left(-p_{0}^{\prime}\right) \delta n^{k l *}(t,-\mathbf{p})\right](2 \pi)^{3} \delta^{3}\left(\mathbf{p}-\mathbf{p}^{\prime}\right) \\
& \times 2 \pi\left(2 \operatorname{sign}\left(p_{0}^{\prime}\right) p_{0}^{\prime}\right)^{1 / 2} \delta\left(\operatorname{det} \boldsymbol{D}_{A}\left(p^{\prime}\right)\right)\left[\operatorname{adj} \boldsymbol{D}_{A}\left(p^{\prime}\right)\right]^{l j} .
\end{aligned}
$$

In order to compare this result directly with the Heisenberg picture, we make use of the pole approximation in eqs. (2.14) and (2.15). For $p_{0}>0$, the complex delta function $2 \pi \delta\left(\operatorname{det} \boldsymbol{D}_{R}\right)$ corresponds to the contribution from the poles at $p_{0}=\Omega_{i}$. Instead, for $p_{0}<0$, the complex delta function $2 \pi \delta\left(\operatorname{det} \boldsymbol{D}_{R}\right)$ corresponds to the contribution from the poles at $p_{0}=-\Omega_{i}^{*}$. Hence, we can write

$$
\begin{aligned}
2 \pi \delta\left(\operatorname{det} \boldsymbol{D}_{R}(p)\right) \approx & \frac{i}{\Delta \Omega^{2}}\left[\frac{1}{2 \Omega_{1}} \frac{f_{1}\left(p_{0}\right)}{p_{0}-\Omega_{1}}-\frac{1}{2 \Omega_{2}} \frac{f_{2}\left(p_{0}\right)}{p_{0}-\Omega_{2}}\right] \\
& -\frac{i}{\Delta \Omega^{* 2}}\left[\frac{1}{2 \Omega_{1}^{*}} \frac{f_{1}^{*}\left(-p_{0}\right)}{p_{0}+\Omega_{1}^{*}}-\frac{1}{2 \Omega_{2}^{*}} \frac{f_{2}^{*}\left(-p_{0}\right)}{p_{0}+\Omega_{2}^{*}}\right],
\end{aligned}
$$

where the $f_{i}\left(p_{0}\right)$ satisfy the properties highlighted above (see eq. (3.36)). An appropriate choice for these functions (see ref. [98]) is $f_{i}\left(p_{0}\right)=\left[\lambda_{i}^{2} /\left(p_{0}^{2}+\lambda_{i}^{2}\right)\right] e^{-i\left(p_{0}-\operatorname{Re} \Omega_{i}\right) / \kappa_{i}}$, where $\kappa_{i} \gg \Gamma_{i} / 2$ and $\lambda_{i} \gg \operatorname{Re} \Omega_{i}>\Gamma_{i}$. The relative sign between the poles at $\Omega_{1}$ and $\Omega_{2}$ arises from the partial-fractioning of $1 /\left[\left(q_{0}^{2}-\Omega_{1}^{2}\right)\left(q_{0}^{2}-\Omega_{2}^{2}\right)\right]$, and the relative sign between the positive- and negative-frequency poles results from the partial-fractioning of $1 /\left(q_{0}^{2}-\Omega_{i}^{2}\right)$. In order to ensure that this partial-fractioning is consistent with the analytic structure of the retarded propagator, we first let $\Omega_{i} \equiv \Omega_{i}\left(q_{0}\right)=-\Omega_{i}^{*}\left(-q_{0}\right)$, before approximating $\Omega_{i}$ in the vicinity of the poles by eq. (2.13). In the limit $\Pi_{R} \rightarrow 0, \kappa_{i} \rightarrow 0$ and $\lambda_{i} \rightarrow \infty$, we recover the standard Dirac delta function:

$$
\begin{array}{r}
\frac{i f_{i}\left(p_{0}\right)}{p_{0}-\Omega_{i}} \longrightarrow 2 \pi \delta\left(p_{0}-E_{i}\right), \\
\frac{i f_{i}^{*}\left(-p_{0}\right)}{p_{0}+\Omega_{i}^{*}} \longrightarrow 2 \pi \delta\left(p_{0}+E_{i}\right) .
\end{array}
$$

In the same limit, we therefore find

$$
\begin{aligned}
2 \pi \delta\left(\operatorname{det} \boldsymbol{D}_{R}(p)\right) & \longrightarrow \frac{2 \pi}{\left|\Delta M^{2}\right|}\left[\frac{\delta\left(p_{0}-E_{1}\right)}{2 E_{1}}-\frac{\delta\left(p_{0}-E_{2}\right)}{2 E_{2}}-\frac{\delta\left(p_{0}+E_{1}\right)}{2 E_{1}}+\frac{\delta\left(p_{0}+E_{2}\right)}{2 E_{2}}\right] \\
& =\frac{2 \pi}{\Delta M^{2}} \operatorname{sign}\left(p_{0}\right)\left[\delta\left(p^{2}-M_{1}^{2}\right)-\delta\left(p^{2}-M_{2}^{2}\right)\right],
\end{aligned}
$$

and

$$
\begin{aligned}
G_{\delta}^{i j}\left(p, p^{\prime}, \tilde{t}\right) \longrightarrow & 2 \pi\left(2 \operatorname{sign}\left(p_{0}\right) p_{0}\right)^{1 / 2} \delta\left(p^{2}-M_{i}^{2}\right) e^{i\left(p_{0}-p_{0}^{\prime}\right) \tilde{t}} \\
& \times\left[\theta\left(p_{0}\right) \theta\left(p_{0}^{\prime}\right) \delta n^{i j}(t, \mathbf{p})+\theta\left(-p_{0}\right) \theta\left(-p_{0}^{\prime}\right) \delta n^{i j *}(t,-\mathbf{p})\right](2 \pi)^{3} \delta^{(3)}\left(\mathbf{p}-\mathbf{p}^{\prime}\right) \\
& \times 2 \pi\left(2 \operatorname{sign}\left(p_{0}^{\prime}\right) p_{0}^{\prime}\right)^{1 / 2} \delta\left(p^{\prime 2}-M_{j}^{2}\right) \\
= & G_{\delta}^{0, i j}\left(p, p^{\prime}, \tilde{t}\right),
\end{aligned}
$$


recovering the non-equilibrium part of the tree-level propagator (cf. eq. (3.1)), as we would expect.

By extracting the positive-frequency part of eq. (3.50), we can define a generalization of eq. (3.7):

$$
\begin{aligned}
2 \pi \delta_{+}\left(\operatorname{det} \boldsymbol{D}_{R}(p)\right) & \equiv \frac{i}{\Delta \Omega^{2}}\left[\frac{1}{2 \Omega_{1}} \frac{f_{1}\left(p_{0}\right)}{p_{0}-\Omega_{1}}-\frac{1}{2 \Omega_{2}} \frac{f_{2}\left(p_{0}\right)}{p_{0}-\Omega_{2}}\right] \\
& \equiv \frac{2 \pi}{\Delta \Omega^{2}}\left[\frac{1}{2 \Omega_{1}} \delta\left(p_{0}-\Omega_{1}\right)-\frac{1}{2 \Omega_{2}} \delta\left(p_{0}-\Omega_{2}\right)\right]
\end{aligned}
$$

We may then write the positive-frequency, non-equilibrium part of the full propagator as

$$
\begin{aligned}
\left.G_{\delta}^{i j}\left(p, p^{\prime}, \tilde{t}\right)\right|_{p_{0}, p_{0}^{\prime}>0}= & 2 \pi\left(2 p_{0}\right)^{1 / 2}\left[\operatorname{adj} \boldsymbol{D}_{R}(p)\right]^{i k} \delta_{+}\left(\operatorname{det} \boldsymbol{D}_{R}(p)\right) e^{i\left(p_{0}-p_{0}^{\prime}\right) \tilde{t}} \\
& \times \delta n^{k l}(t, \mathbf{p})(2 \pi)^{3} \delta^{3}\left(\mathbf{p}-\mathbf{p}^{\prime}\right) 2 \pi\left(2 p_{0}^{\prime}\right)^{1 / 2} \delta_{+}\left(\operatorname{det} \boldsymbol{D}_{A}\left(p^{\prime}\right)\right)\left[\operatorname{adj} \boldsymbol{D}_{A}\left(p^{\prime}\right)\right]^{l j},
\end{aligned}
$$

where

$$
\delta_{+}\left(\operatorname{det} \boldsymbol{D}_{A}(p)\right)=\left[\delta_{+}\left(\operatorname{det} \boldsymbol{D}_{R}(p)\right)\right]^{*} .
$$

We note that eqs. (3.50) and (3.54) cannot be applied naively in the doubly-degenerate case, where the limit $\Omega_{2} \rightarrow \Omega_{1}$ must be taken before the integral over $p_{0}$. It is in the use of the pole approximation in eq. (2.14) that the present analysis differs from that of ref. [11], where instead an alternative procedure was employed based upon the resummation techniques developed in ref. [35]. We note however that the approximation used there (see appendix A.1 of ref. [11]) cannot be used in the weak-washout regime, where one cannot guarantee that the off-diagonal number densities are of $\mathcal{O}\left(h^{2}\right)$, as is the case for the strongwashout regime considered in ref. [11].

In the equal-time limit $X^{0}=\tilde{t}$, and using eq. (3.54), the Wigner transform of eq. (3.55) is

$$
G_{\delta}^{i j}\left(t, q_{0}>0\right)=2 \pi \delta\left(q_{0}-\Omega_{a b}\right)\left[\operatorname{adj} \boldsymbol{D}_{R}\left(\Omega_{a}\right)\right]^{i k} \frac{\epsilon_{a b} \delta n^{k l}(t, \mathbf{q})}{\left(2 \Omega_{a}\right)^{1 / 2}\left(2 \Omega_{b}^{*}\right)^{1 / 2}\left|\Delta \Omega^{2}\right|^{2}}\left[\operatorname{adj} \boldsymbol{D}_{A}\left(\Omega_{b}^{*}\right)\right]^{l j}
$$

Here, the sum over $a, b=1,2$ has been left implicit, $\Omega_{a b} \equiv\left(\Omega_{a}+\Omega_{b}^{*}\right) / 2$, and $\epsilon_{a b}=1$ if $a=b$ and $\epsilon_{a b}=-1$ if $a \neq b$. Finally, performing the summations over $a$ and $b$, we find

$$
\begin{aligned}
G_{\delta}^{i j}\left(t, q_{0}>0\right)= & 2 \pi \delta\left(q_{0}-\omega_{1}\right)\left[\operatorname{adj} \boldsymbol{D}_{R}\left(\Omega_{1}\right)\right]^{i k} \frac{\delta n^{k l}(t, \mathbf{q})}{\left|2 \Omega_{1}\right|\left|\Delta \Omega^{2}\right|^{2}}\left[\operatorname{adj} \boldsymbol{D}_{A}\left(\Omega_{1}^{*}\right)\right]^{l j} \\
& +2 \pi \delta\left(q_{0}-\omega_{2}\right)\left[\operatorname{adj} \boldsymbol{D}_{R}\left(\Omega_{2}\right)\right]^{i k} \frac{\delta n^{k l}(t, \mathbf{q})}{\left|2 \Omega_{2}\right|\left|\Delta \Omega^{2}\right|^{2}}\left[\operatorname{adj} \boldsymbol{D}_{A}\left(\Omega_{2}^{*}\right)\right]^{l j} \\
& -2 \pi \delta\left(q_{0}-\bar{\Omega}\right)\left[\operatorname{adj} \boldsymbol{D}_{R}\left(\Omega_{1}\right)\right]^{i k} \frac{\delta n^{k l}(t, \mathbf{q})}{\left(2 \Omega_{1}\right)^{1 / 2}\left(2 \Omega_{2}^{*}\right)^{1 / 2}\left|\Delta \Omega^{2}\right|^{2}}\left[\operatorname{adj} \boldsymbol{D}_{A}\left(\Omega_{2}^{*}\right)\right]^{l j} \\
& -2 \pi \delta\left(q_{0}-\bar{\Omega}^{*}\right)\left[\operatorname{adj} \boldsymbol{D}_{R}\left(\Omega_{2}\right)\right]^{i k} \frac{\delta n^{k l}(t, \mathbf{q})}{\left(2 \Omega_{1}^{*}\right)^{1 / 2}\left(2 \Omega_{2}\right)^{1 / 2}\left|\Delta \Omega^{2}\right|^{2}}\left[\operatorname{adj} \boldsymbol{D}_{A}\left(\Omega_{1}^{*}\right)\right]^{l j}
\end{aligned}
$$


where $\bar{\Omega}=\left(\Omega_{1}+\Omega_{2}^{*}\right) / 2$. It is essential to emphasize that the deviations from equilibrium $\delta n^{i j}$ are the non-equilibrium parts of the physical dynamical number densities, which appear as unknowns in the interaction-picture propagators. Moreover, these are the spectrallyfree number densities, which count excitations with energy $E_{i}$.

In order to compare with the Heisenberg-picture result in eq. (2.19), we now expand the interaction-picture result in eq. (3.58) above to first order in $\Pi_{R(A)}$. This gives the following result for the off-diagonal components:

$$
\begin{aligned}
& G_{\delta}^{i t}\left(t, q_{0}>0\right) \approx 2 \pi \delta\left(q_{0}-\omega_{i}\right) \frac{1}{2 \omega_{i}} \delta n^{i i}(t) \Pi_{A}^{i t}\left(\omega_{i}\right) R_{i \neq} \\
& -2 \pi \delta\left(q_{0}-\omega_{i}\right) \frac{1}{2 \omega_{i}} \delta n^{i t}(t) \Pi_{R}^{i t}\left(\omega_{i}\right) R_{i \neq} \\
& +2 \pi \delta\left(q_{0}-\bar{\omega}\right) \frac{1}{\left(2 \omega_{i}\right)^{1 / 2}\left(2 \omega_{i}\right)^{1 / 2}}\left[\delta n^{i \not}(t) \Delta M_{i \neq}^{2}\right. \\
& \left.-\delta n^{i i}(t) \Pi_{A}^{i t}\left(\omega_{i}\right)+\delta n^{i t}(t) \Pi_{R}^{i t}\left(\omega_{i}\right)\right] R_{i t} .
\end{aligned}
$$

Thus, we find for the time-derivative of the asymmetry

$$
\begin{aligned}
\frac{\mathrm{d} \eta}{\mathrm{d} t} \approx 2 \sum_{i} \int_{\mathbf{q}} \frac{M_{i}}{\omega_{i}} \delta n^{i i}(t, \mathbf{q}) \Gamma_{i}^{\mathrm{med}}\left(\omega_{i}, \mathbf{q}\right) \epsilon_{i}^{\mathrm{med}}\left(\omega_{i}, \mathbf{q}\right) \\
+2 \operatorname{Im} H_{12} \operatorname{Im} \int_{\mathbf{q}} \frac{\widetilde{\Pi}_{\rho}(\bar{\omega}, \mathbf{q})}{\left(\omega_{1} \omega_{2}\right)^{\frac{1}{2}}}\left[\delta n^{12}(t, \mathbf{q}) \Delta M_{12}^{2}\right. \\
\\
\left.\quad-\delta n^{11}(t, \mathbf{q}) \Pi_{A}^{12}\left(\omega_{2}, \mathbf{q}\right)+\delta n^{22}(t, \mathbf{q}) \Pi_{R}^{12}\left(\omega_{1}, \mathbf{q}\right)\right] R_{12} .
\end{aligned}
$$

This closely resembles the Heisenberg-picture result in eq. (2.28) with the exception of the time-dependent phases; the Heisenberg-picture result is written in terms of the initial conditions for the non-equilibrium parts of the number densites. Hence, in order to show that the expressions for $G_{\delta}^{i j}$ in eqs. (2.19) and (3.59) are in fact identical to first order in the self-energies, and by extension the expressions for the asymmetry in eqs. (3.60) and (2.28), we must now find the functional form of $\delta n^{i j}(t)$ by solving the transport equations directly. This will allow us to write the interaction-picture result directly in terms of the initial conditions.

Before proceeding to do this, however, it is important to remark upon the role played by the interference terms. These interference terms may now be distinguished from the pure oscillation contribution. The former appear in the final line of eq. (3.60) and originate in the final line of eq. (3.59), lying on the oscillation shell but being proportional to the diagonal components of the time-dependent number densities. On the other hand, the oscillation contribution appears in the second line of eq. (3.60) and originates in third line of eq. (3.59), and is proportional to the off-diagonal components of the time-dependent number densities. This identification of the pure oscillation contribution is in accord with the conventions of refs. [9-11] and, as we will see below, it is subtly different to identifying the pure oscillation and interference contributions in terms of the components of the initial deviations from equilibrium, as appear in eq. (2.19), which makes sense only in the weakwashout regime. Note that, although it is possible to identify the mixing and oscillation 
shells unambiguously by means of the spectral structure of the resummed propagators, it is possible to identify the pure oscillation and interference terms only through a physicallymeaningful definition of the particle number densities.

We proceed by expanding all but the regulator structure in eq. (3.59) around $\Delta \omega_{i j}=$ $\omega_{i}-\omega_{i}=0$. At zeroth order, we find

$$
G_{\delta}^{i \not}\left(t, q_{0}>0\right) \supset 2 \pi \delta\left(q_{0}-\bar{\omega}\right) \frac{1}{2 \bar{\omega}} \delta n^{i \not}(t) \Delta M_{i \downarrow}^{2} R_{i \downarrow},
$$

in which the mixing contributions have canceled and from which we see that the interference between mixing and oscillations is destructive. At this point, one might be tempted to conclude that using the on-shell approximation for the heavy-neutrino propagator, $\mathrm{cf}$. eq. (3.11), in the equation for the asymmetry is valid, and therefore that the approach of refs. [9-11], by including contributions from both mixing and oscillation, double-counts the final asymmetry. However, this is not the case. Continuing to the next order in the expansion, we find

$$
\begin{aligned}
& G_{\delta}^{i \ell}(t, q) \approx 2 \pi \delta\left(q_{0}-\bar{\omega}\right) \frac{1}{2 \bar{\omega}} \delta n^{i \hbar}(t) \Delta M_{i j}^{2} R_{i \not} \\
& -2 \pi \delta\left(q_{0}-\bar{\omega}\right) \frac{1}{2 \bar{\omega}}\left[\delta n^{i i}(t) \frac{\Pi_{A}^{i \hbar}(\bar{\omega})}{4 \bar{\omega}^{2}}+\delta n^{i t}(t) \frac{\Pi_{R}^{i \bar{t}}(\bar{\omega})}{4 \bar{\omega}^{2}}\right] \Delta M_{i \bar{t}}^{2} R_{i \neq},
\end{aligned}
$$

where the mixing terms are present but are now suppressed by an additional factor of $\Delta M_{12}^{2}$. Here, we have neglected terms proportional to the derivative of the delta function $\delta^{\prime}\left(q_{0}-\bar{\omega}\right)$ and the derivative of the self-energy $\Pi^{i \not{ }^{\prime}}(\bar{\omega})$, which contribute sub-dominantly to the asymmetry under the assumption that the self-energies are slowly varying functions of $q_{0}$ for $q_{0} \sim \bar{\omega}$. This same assumption underlies the pole approximations in eqs. (2.14) and (2.15), which can be verified numerically (see ref. [72]). The asymmetry now takes the form

$$
\begin{aligned}
\frac{\mathrm{d} \eta}{\mathrm{d} t} \approx & 2 \sum_{i} \int_{\mathbf{q}} \frac{M_{i}}{\bar{\omega}} \delta n^{i i}(t, \mathbf{q}) \Gamma_{i}^{\operatorname{med}}(\bar{\omega}, \mathbf{q}) \tilde{\epsilon}_{i}^{\operatorname{med}}(\bar{\omega}, \mathbf{q}) \\
& +2 \operatorname{Im} H_{12} \int_{\mathbf{q}} \frac{\widetilde{\Pi}_{\rho}(\bar{\omega}, \mathbf{q})}{\bar{\omega}} \operatorname{Im} \delta n^{12}(t, \mathbf{q}) \Delta M_{12}^{2} R_{12},
\end{aligned}
$$

where the usual $C P$-violating parameter has been modified:

$$
\tilde{\epsilon}_{i}^{\operatorname{med}}(\bar{\omega}, \mathbf{q})=\frac{\omega_{p}-\omega_{i}}{\omega_{k}+\omega_{i}} \epsilon_{i}^{\operatorname{med}}(\bar{\omega}, \mathbf{q}),
$$

cf. eq. (2.29). Although both mixing and oscillation contributions persist in the middle-shell approximation and are clearly identifiable, in agreement with the results of refs. [9-11], we see that the structure of the mixing contribution has been modified. In addition to the suppression by an additional factor of $\Delta M_{12}^{2}$, a relative sign has emerged between the $i=1$ and $i=2$ contributions. As a result, the modified mixing contribution is strongly suppressed when the deviations of the number densities of the two flavours from equilibrium are similar. However, this is not always the case, for instance in scenarios of resonant $\ell$ genesis $[19,20]$ (see also refs. [9-11]), where the lepton asymmetry is dominantly produced 
in a single flavour through the decays of heavy neutrinos of a particular family type. In such cases, both mixing and oscillation contributions will be present. Most significantly, we observe from eqs. (3.62) and (3.63) that the pre-factors of these two distinct contributions to the lepton asymmetry carry exactly the same parametric dependence on the Yukawa couplings and mass splittings: $\sim \Delta M_{12}^{2} R_{12}$, which is of order unity in the weakly-resonant or overlapping regime $\Gamma_{i} \ll \Delta M \ll \bar{M}$. Finally, we remark that it remains to be seen how the mixing source is modified by interference in the case of more than two flavours.

Explicit solution. We now return to eq. (3.58) with the aim of finding the explicit solution for the deviations from equilibrium $\delta n^{i j}(t)$. The relevant transport equations for determining the functional form of the spectrally-dressed number densities $\delta n_{\mathrm{dr}}^{i j}(t)$ can be written in the general form [11]

$$
\begin{aligned}
\frac{\mathrm{d} \delta n_{\mathrm{dr}}^{i j}(t)}{\mathrm{d} t}= & \int \frac{\mathrm{d} p_{0}}{2 \pi} \int \frac{\mathrm{d} p_{0}^{\prime}}{2 \pi} e^{-i\left(p_{0}-p_{0}^{\prime}\right) \tilde{t}} \theta\left(p_{0}\right) \theta\left(p_{0}^{\prime}\right) \\
& \times\left(-i\left[\boldsymbol{M}^{2}+\operatorname{Re} \boldsymbol{\Pi}_{R}, \boldsymbol{G}_{\delta}\right]_{\star}^{i j}-\frac{1}{2}\left\{\boldsymbol{\Pi}_{<}, \boldsymbol{G}_{\delta}\right\}_{\star}^{i j}+\frac{1}{2}\left\{\boldsymbol{\Pi}_{>}, \boldsymbol{G}_{\delta}\right\}_{\star}^{i j}\right) .
\end{aligned}
$$

In the Markovian approximation, the commutators and anti-commutators appearing in eq. (3.65) are defined as follows [11]:

$$
\begin{aligned}
{[\boldsymbol{A}, \boldsymbol{B}]_{\star} } & \equiv \int_{k}\left(\boldsymbol{A}(p, k) \cdot \boldsymbol{B}\left(k, p^{\prime}\right)-\boldsymbol{B}(p, k) \cdot \boldsymbol{A}\left(k, p^{\prime}\right)\right), \\
\{\boldsymbol{A}, \boldsymbol{B}\}_{\star} & \equiv \int_{k}\left(\boldsymbol{A}(p, k) \cdot \boldsymbol{B}\left(k, p^{\prime}\right)+\boldsymbol{B}(p, k) \cdot \boldsymbol{A}\left(k, p^{\prime}\right)\right),
\end{aligned}
$$

in which we emphasize the order of the four-momenta.

In order to find the asymmetry at first order in $\Pi_{R(A)}$, we require the solution for the diagonals only at zeroth order. Thus, for the diagonals, we may work in terms of the treelevel $G_{\delta}^{0}$, as obtained from eq. (3.1), yielding the following equation for the spectrally-free number densities

$$
\frac{\mathrm{d} \delta n^{i i}}{\mathrm{~d} t}=-\Gamma_{i} \delta n^{i i}
$$

with solution

$$
\delta n^{i i}(t)=e^{-\Gamma_{i} t} \delta n^{i i}(0) .
$$

For the off-diagonals, we may work in terms of the tree-level $G_{\delta}^{0}$ in the time-derivative on the l.h.s. and in all terms already at leading order in $\Pi_{R(A)}$ on the r.h.s., i.e.

$$
\begin{aligned}
\frac{\mathrm{d} \delta n^{i \ell}(t)}{\mathrm{d} t} \supset & \int \frac{\mathrm{d} p_{0}}{2 \pi} \int \frac{\mathrm{d} p_{0}^{\prime}}{2 \pi} e^{-i\left(p_{0}-p_{0}^{\prime}\right) \tilde{t}} \theta\left(p_{0}\right) \theta\left(p_{0}^{\prime}\right) \\
& \times\left(-i\left[\operatorname{Re} \boldsymbol{\Pi}_{R}, \boldsymbol{G}_{\delta}^{0}\right]_{\star}^{i \neq}-\frac{1}{2}\left\{\boldsymbol{\Pi}_{<}, \boldsymbol{G}_{\delta}^{0}\right\}_{\star}^{i \not}+\frac{1}{2}\left\{\boldsymbol{\Pi}_{>}, \boldsymbol{G}_{\delta}^{0}\right\}_{\star}^{i \not}\right) .
\end{aligned}
$$

On the other hand, in the term

$$
\frac{\mathrm{d} \delta n^{i \not t}(t)}{\mathrm{d} t} \supset-i \int \frac{\mathrm{d} p_{0}}{2 \pi} \int \frac{\mathrm{d} p_{0}^{\prime}}{2 \pi} e^{-i\left(p_{0}-p_{0}^{\prime}\right) \tilde{t}} \theta\left(p_{0}\right) \theta\left(p_{0}^{\prime}\right)\left[\boldsymbol{M}^{2}, \boldsymbol{G}_{\delta}\right]_{\star}^{i \hbar},
$$


we must use the resummed $\boldsymbol{G}_{\delta}$, since this will also contribute a term at first order in $\Pi_{R(A)}$. Proceeding in this manner, we find the equation for the off-diagonals

$$
\frac{\mathrm{d} \delta n^{i \not}}{\mathrm{d} t}=-i\left(\omega_{i}-\omega_{i}\right) \delta n^{i \hbar}-\bar{\Gamma} \delta n^{i \hbar}-\frac{i}{2 \bar{\omega}}\left(\Pi_{R}^{i t}\left(\omega_{i}\right) \delta n^{\not \hbar}-\Pi_{A}^{i t}\left(\omega_{\ell}\right) \delta n^{i i}\right),
$$

in which we have used the approximation $\omega_{i} \omega_{i} \approx \bar{\omega}^{2}$.

It is interesting to remark upon the origin of the order $\Pi_{R(A)}$ terms in eq. (3.71). The terms originating from the diagonal elements of the tree-level $\boldsymbol{G}_{\delta}^{0}$ in eq. (3.69) are

$$
\frac{\mathrm{d} \delta n^{i \not}}{\mathrm{d} t} \supset-\frac{i}{2 \bar{\omega}} \delta n^{i \hbar} \Pi_{R}^{i t}\left(\omega_{i}\right)+\frac{i}{2 \bar{\omega}} \delta n^{i i} \Pi_{A}^{i \hbar}\left(\omega_{i}\right) .
$$

Instead, the off-diagonal element of the resummed $\boldsymbol{G}_{\delta}$ used in the commutator in eq. (3.70) yields the following terms:

$$
\frac{\mathrm{d} \delta n^{i \not t}}{\mathrm{~d} t} \supset-\frac{i}{2 \bar{\omega}} \delta n^{i i}\left(\Pi_{A}^{i t}\left(\omega_{i}\right)-\Pi_{A}^{i t}\left(\omega_{i}\right)\right)+\frac{i}{2 \bar{\omega}} \delta n^{\not t}\left(\Pi_{R}^{i \not}\left(\omega_{i}\right)-\Pi_{R}^{i t}\left(\omega_{i}\right)\right) .
$$

We see that the contribution from the resummed $\boldsymbol{G}_{\delta}$ in eq. (3.70), which amounts to the interference of mixing and oscillation effects, swaps the arguments of the terms at first order in $\Pi_{R(A)}$.

The leading-order solutions to the off-diagonal equations in eq. (3.71) have the form

$$
\delta n^{i \ell}(t)=e^{-i\left(\omega_{i}-\omega_{i}\right) t} e^{-\bar{\Gamma} t} \delta n_{0}^{i \not}(0)+\frac{\Pi_{A}^{i \hbar}\left(\omega_{i}\right)}{\Delta M_{i \not}^{2}} e^{-\Gamma_{i} t} \delta n^{i i}(0)-\frac{\Pi_{R}^{i \hbar}\left(\omega_{i}\right)}{\Delta M_{i \not}^{2}} e^{-\Gamma_{i} t} \delta n^{\not k}(0),
$$

where $\delta n_{0}^{i f}$ is the initial condition at zeroth order in $\Pi_{R(A)}$. Next, we re-express this result in terms of the full initial condition for the off-diagonals $\delta n^{i t}(0)$ :

$$
\delta n^{i t}(0)=\delta n_{0}^{i t}(0)+\frac{\Pi_{A}^{i t}\left(\omega_{i}\right)}{\Delta M_{i \neq}^{2}} \delta n^{i i}(0)-\frac{\Pi_{R}^{i t}\left(\omega_{i}\right)}{\Delta M_{i \neq}^{2}} \delta n^{i t}(0) .
$$

We then obtain the final form of the solution:

$$
\begin{aligned}
\delta n^{i \not}(t)= & e^{-i\left(\omega_{i}-\omega_{i}\right) t} e^{-\bar{\Gamma} t} \delta n^{i \hbar}(0) \\
& +\frac{\Pi_{A}^{i j}\left(\omega_{i}\right)}{\Delta M_{i \not}^{2}}\left(e^{-\Gamma_{i} t}-e^{-i\left(\omega_{i}-\omega_{i}\right) t} e^{-\bar{\Gamma} t}\right) \delta n^{i i}(0) \\
& -\frac{\Pi_{R}^{i \not}\left(\omega_{i}\right)}{\Delta M_{i \not l}^{2}}\left(e^{-\Gamma_{i} t}-e^{-i\left(\omega_{i}-\omega_{i}\right) t} e^{-\bar{\Gamma} t}\right) \delta n^{i \not t}(0) .
\end{aligned}
$$

Substituting eq. (3.76) into eq. (3.59) for the off-diagonal components of $\boldsymbol{G}_{\delta}$, we obtain precisely eq. (2.19), as derived in the Heisenberg picture. This remarkable agreement provides a significant illustration of both the self-consistency and complementarity of the Heisenberg- and interaction-picture approaches described in this article.

Finally, we see from eq. (3.76) the reason for defining the pure oscillation and interference terms with respect to the time-dependent number densities in eqs. (3.59) and (3.60) 
and not with respect to their initial conditions, as appear in eqs. (2.19) and (2.28). In the weak-washout regime treated here, the explicit solution for the off-diagonals $\delta n^{i t}(t)$ in eq. (3.76) contains terms proportional to the initial deviation of the diagonal components $\delta n^{i i}(0)$ and $\delta n^{i t}(0)$, which appear comparable to the terms in the final line of eqs. (3.59) and (3.60). On the other hand, in the strong-washout regime, whilst the solution for the off-diagonals $\delta n^{i t}(t)$ would no longer depend on the initial conditions, the final line in eq. (3.60) would still be present.

\section{Comparison with the density matrix approximation}

In the two previous sections, we have paid particular attention to the shell structure of the would-be heavy-neutrino propagators and emphasized the need to keep track of this complete structure in the equation for the asymmetry. However, in phenomenological studies and in order to obtain the final lepton asymmetry, we need first to solve the evolution equations for the number densities of the heavy neutrinos. In contrast to the equation for the asymmetry, for an almost degenerate mass spectrum, there is no need to keep track of the different shells in the evolution of the heavy-neutrino number densities. Such a single shell approximation applied to the heavy-neutrino transport equations will be referred to as the density matrix approximation in what follows. In the Kadanoff-Baym formalism, this apparent disparity between the treatment of the equation for the asymmetry and the evolution equations of the heavy-neutrino number densities is actually a result of making a self-consistent loop-wise perturbative truncation of the leptonic and heavy-neutrino KB equations (see refs. [11] and [84]).

Performing a Wigner-transform of the Kadanoff-Baym equations (eq. (2.3)) and neglecting the gradient terms, one arrives at the following equation for the Wigner transform of the Wightman propagators [7]:

$$
2 q_{0} \partial_{t} \boldsymbol{G}_{<}+i\left[\boldsymbol{M}^{2}, \boldsymbol{G}_{<}\right]=\frac{1}{2}\left\{\boldsymbol{\Pi}_{>}, \boldsymbol{G}_{<}\right\}-\frac{1}{2}\left\{\boldsymbol{\Pi}_{<}, \boldsymbol{G}_{>}\right\},
$$

where the term $\operatorname{Re} \boldsymbol{\Pi}_{R}$ (see eq. (3.65)) is understood to have been absorbed into the mass matrix $\boldsymbol{M}^{2}$. In the density matrix approximation [7, 59],

$$
\boldsymbol{G}_{<}(t, q)=\boldsymbol{n}(t, \mathbf{q}) 2 \pi \delta\left(q^{2}-\bar{M}^{2}\right)
$$

where $\bar{M}^{2}=\left(M_{1}^{2}+M_{2}^{2}\right) / 2$ and the density matrix (or, to be more precise, the matrix of densities) $\boldsymbol{n}(t, \mathbf{q})$ can be viewed as a dressed distribution function. Substituting eq. (4.2) into eq. (4.1), we arrive at

$$
2 q_{0} \partial_{t} \boldsymbol{n}+i\left[\boldsymbol{M}^{2}, \boldsymbol{n}\right]=\frac{1}{2}\left\{\boldsymbol{\Pi}_{>}, \boldsymbol{n}\right\}-\frac{1}{2}\left\{\boldsymbol{\Pi}_{<}, \mathbf{1}+\boldsymbol{n}\right\} .
$$

Finally, integrating over $q_{0}$ and using the single-shell approximation in eq. (4.2), we obtain the kinetic equation for the matrix of densities

$$
\partial_{t} \boldsymbol{n}+\frac{i}{2 \bar{\omega}}\left[\boldsymbol{M}^{2}, \boldsymbol{n}\right]=\frac{1}{4 \bar{\omega}}\left\{\boldsymbol{\Pi}_{>}, \boldsymbol{n}\right\}-\frac{1}{4 \bar{\omega}}\left\{\boldsymbol{\Pi}_{<}, \mathbf{1}+\boldsymbol{n}\right\} .
$$


In equilibrium, $\boldsymbol{n}$ is time independent and, as follows from eq. (4.4), satisfies

$$
2 i\left[\boldsymbol{M}^{2}, \boldsymbol{n}_{\mathrm{eq}}\right]=\left\{\boldsymbol{\Pi}_{>}, \boldsymbol{n}_{\mathrm{eq}}\right\}-\left\{\boldsymbol{\Pi}_{<}, \mathbf{1}+\boldsymbol{n}_{\mathrm{eq}}\right\} .
$$

The Kubo-Martin-Schwinger relation (see e.g. ref. [100]) implies that $\boldsymbol{\Pi}_{<}=\widetilde{\boldsymbol{\Pi}}_{\rho} n_{\mathrm{BE}}$ in equilibrium, where $n_{\mathrm{BE}}$ is the Bose-Einstein distribution function. Further, taking into account that $\widetilde{\boldsymbol{\Pi}}_{\rho}=\boldsymbol{\Pi}_{>}-\boldsymbol{\Pi}_{<}$, we conclude that $\boldsymbol{n}_{\mathrm{eq}}=\mathbf{1} \cdot n_{\mathrm{BE}}$ in the density matrix approximation, i.e. that the equilibrium distribution function is diagonal and, as expected, given by the Bose-Einstein distribution.

Writing $\boldsymbol{n}=\boldsymbol{n}_{\mathrm{eq}}+\delta \boldsymbol{n}$ and using eqs. (4.4) and (4.5), we obtain the kinetic equation for the deviation of the matrix of densities from its equilibrium value

$$
\partial_{t} \delta \boldsymbol{n}+\frac{i}{2 \bar{\omega}}\left[\boldsymbol{M}^{2}, \delta \boldsymbol{n}\right]=\frac{1}{4 \bar{\omega}}\left\{\widetilde{\boldsymbol{\Pi}}_{\rho}, \delta \boldsymbol{n}\right\}
$$

Finally, introducing the matrix of effective decay widths

$$
\boldsymbol{\Gamma} \equiv-\frac{\widetilde{\boldsymbol{\Pi}}_{\rho}}{2 \bar{\omega}}=\frac{i \boldsymbol{\Pi}_{\rho}}{2 \bar{\omega}}
$$

and using the standard trick $M^{2} \rightarrow 2 \bar{\omega} \boldsymbol{\omega}$, we arrive at the equation

$$
\partial_{t} \delta \boldsymbol{n}+i[\boldsymbol{\omega}, \delta \boldsymbol{n}]=-\frac{1}{2}\{\boldsymbol{\Gamma}, \delta \boldsymbol{n}\}
$$

as obtained in ref. [7].

It can readily be checked by substitution that the solution to eq. (4.8) is

$$
\delta \boldsymbol{n}(t)=e^{-i\left(\boldsymbol{\omega}-\frac{i}{2} \boldsymbol{\Gamma}\right) t} \delta \boldsymbol{n}(0) e^{i\left(\boldsymbol{\omega}+\frac{i}{2} \boldsymbol{\Gamma}\right) t} .
$$

Choosing, as in ref. [7], initial conditions of the form

$$
\delta \boldsymbol{n}(0)=\left(\begin{array}{cc}
\delta n^{11}(0) & 0 \\
0 & 0
\end{array}\right)
$$

we obtain, to leading order in $\boldsymbol{\Gamma}$,

$$
\delta n^{i \hbar}(t) \approx \frac{i}{2} \frac{\Gamma^{i \not}}{\left(\omega_{i}-\omega_{i}\right)+\frac{i}{2}\left(\Gamma_{i}-\Gamma_{\ell}\right)} \delta n^{11}(0)\left(e^{-\Gamma_{i} t}-e^{-i\left(\omega_{i}-\omega_{i}\right) t} e^{-\bar{\Gamma} t}\right),
$$

which is in exact agreement with the result of ref. [7]. For general initial conditions, eq. (4.11) takes the form

$$
\begin{aligned}
\delta n^{i \ell}(t) \approx & \delta n^{i \hbar}(0) e^{-i\left(\omega_{i}-\omega_{i}\right) t} e^{-\bar{\Gamma} t} \\
& +\frac{i}{2} \frac{\Gamma^{i \hbar}}{\left(\omega_{i}-\omega_{i}\right)+\frac{i}{2}\left(\Gamma_{i}-\Gamma_{i}\right)} \delta n^{i i}(0)\left(e^{-\Gamma_{i} t}-e^{-i\left(\omega_{i}-\omega_{i}\right) t} e^{-\bar{\Gamma} t}\right) \\
& +\frac{i}{2} \frac{\Gamma^{i t}}{\left(\omega_{i}-\omega_{i}\right)+\frac{i}{2}\left(\Gamma_{\ell}-\Gamma_{i}\right)} \delta n^{\not t}(0)\left(e^{-\Gamma_{i} t}-e^{-i\left(\omega_{i}-\omega_{i}\right) t} e^{-\bar{\Gamma} t}\right) .
\end{aligned}
$$


Multiplying this expression by $2 \bar{\omega}$ and using again eq. (4.7), we find

$$
\begin{aligned}
\delta n^{i \hbar}(t) \approx & \delta n^{i \hbar}(0) e^{-i\left(\omega_{i}-\omega_{i}\right) t} e^{-\bar{\Gamma} t} \\
& -\frac{i}{2} \frac{\widetilde{\Pi}_{\rho}^{i f}}{\Delta M_{i \downarrow}^{2}} \delta n^{i i}(0)\left(e^{-\Gamma_{i} t}-e^{-i\left(\omega_{i}-\omega_{i}\right) t} e^{-\bar{\Gamma} t}\right) \\
& -\frac{i}{2} \frac{\widetilde{\Pi}_{\rho}^{i f}}{\Delta M_{i \downarrow}^{2}} \delta n^{i \hbar}(0)\left(e^{-\Gamma_{i} t}-e^{-i\left(\omega_{i}-\omega_{i}\right) t} e^{-\bar{\Gamma} t}\right),
\end{aligned}
$$

which, in the density matrix approximation, is identical to eq. (3.76). In other words, for an almost degenerate mass spectrum, one can safely use the density matrix equations to compute the number density of the heavy neutrinos.

\section{Comparison with the effective Yukawa approach}

As was emphasized in the preceding sections, it is necessary to account for the shell structure at the level of the equation for the asymmetry for all mass spectra. This is necessary in order to capture the effect of mixing. In this section, we will compare the result from the present analysis with that of the semi-classical analysis of refs. [9, 10] and the KB analysis of ref. [11]. Therein, the effect of oscillations is captured by accounting for the dynamics of flavour coherences, encoded in the off-diagonal elements of the number density. The effect of mixing is accounted for by means of effective Yukawa couplings, following the work of refs. $[15,35]$. We briefly review the derivation of these effective Yukawa couplings for the present toy model below, as were treated in ref. [58]. We reiterate that this approach was not followed in the preceding sections.

The heavy mixing scalars are unstable, and as such they cannot appear as asymptotic in- or out-states of $S$-matrix elements. Instead, their properties are defined by $S$-matrix elements for scattering of stable particles, $b$ and $\bar{b}$, mediated by the unstable ones [101]. Resumming the propagator of the intermediate heavy states, we can represent two-body scattering processes as a sum of resonant and non-resonant contributions. The $C P$-violating part of the resonant contribution can then be interpreted as a characteristic of the on-shell intermediate particle $[15,102]$.

The amplitude of the $s$-channel two-body scattering process $b b \rightarrow \bar{b} \bar{b}$ can be expressed as

$$
\mathcal{M}_{b b \rightarrow \bar{b} \bar{b}}=\sum_{i, j} \Gamma_{i}^{A} G^{i j}(s) \Gamma_{j}^{B}
$$

where $\Gamma_{i}^{A}$ and $\Gamma_{j}^{B}$ represent the vertices $\psi_{i} b b$ and $\psi_{j} \bar{b} \bar{b}$, including the wave functions of the initial and final states, and $G^{i j}$ are the full vacuum propagators obtained by resumming an infinite series of self-energy graphs [15]. The resummation can be performed using the Schwinger-Dyson equation in vacuum:

$$
\left[G^{-1}\right]^{i j}\left(p^{2}\right)=\left[p^{2}-M_{i}^{2}\right] \delta^{i j}-\Pi^{i j}\left(p^{2}\right)
$$


At one-loop level and in the on-shell scheme, the renormalized self-energy $\Pi^{i j}$ is given by $[58]$

$$
\begin{aligned}
\Pi_{\mathrm{ren}}^{i i} & =\frac{H_{i i}}{16 \pi^{2}}\left[\ln \frac{\left|p^{2}\right|}{M_{i}^{2}}-\frac{p^{2}-M_{i}^{2}}{M_{i}^{2}}-i \pi \theta\left(p^{2}\right)\right] \\
\Pi_{\mathrm{ren}}^{i j} & =\frac{\operatorname{Re} H_{i \not}}{16 \pi^{2}}\left[\frac{p^{2}-M_{i}^{2}}{M_{\not{l}}^{2}-M_{i}^{2}} \ln \frac{\left|p^{2}\right|}{M_{\not{l}}^{2}}+\frac{p^{2}-M_{\not{l}}^{2}}{M_{i}^{2}-M_{\not{l}}^{2}} \ln \frac{\left|p^{2}\right|}{M_{i}^{2}}-i \pi \theta\left(p^{2}\right)\right] .
\end{aligned}
$$

Inverting eq. (5.2), we obtain the following result for the components of the renormalized resummed propagator:

$$
\begin{aligned}
G^{i i}\left(p^{2}\right) & =+\left[G^{-1}\right]^{j j}\left(p^{2}\right) / \operatorname{det}\left[\boldsymbol{G}^{-1}\left(p^{2}\right)\right], \\
G^{i t}\left(p^{2}\right) & =-\left[G^{-1}\right]^{i t}\left(p^{2}\right) / \operatorname{det}\left[\boldsymbol{G}^{-1}\left(p^{2}\right)\right] .
\end{aligned}
$$

Because of the presence of absorptive terms in eq. (5.3), the determinant of the inverse propagator in eq. (5.4) has two poles in the complex plane at

$$
s_{i} \simeq M_{i}^{2}-i M_{i} \Gamma_{i},
$$

where $\Gamma_{i}=H_{i i} / 16 \pi M_{i}$ is the tree-level decay width of $\psi_{i}$. Expanding eq. (5.4) around the poles and substituting the leading expansion terms into eq. (5.1), we find [35]

$$
\mathcal{M}_{b b \rightarrow \bar{b} \bar{b}} \simeq \sum_{i} V_{i}^{A}(s) \frac{1}{s-s_{i}} V_{i}^{B}(s)
$$

where

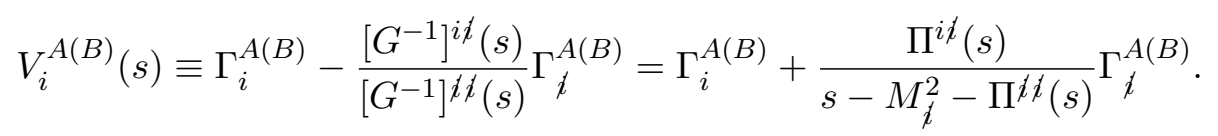

Equation (5.7) can be used to define effective one-loop Yukawa couplings $\mathbf{h}_{i}^{(c)}$. Taking into account that the couplings in the vertices $\psi_{i} b b$ and $\psi_{i} \bar{b} \bar{b}$ differ by complex conjugation, we obtain

$$
\begin{aligned}
\mathbf{h}_{i} & \equiv h_{i}+\frac{\Pi^{i \not}(s)}{s-M_{\not}^{2}-\Pi^{i t}(s)} h_{\not}, \\
\mathbf{h}_{i}^{c *} & \equiv h_{i}^{*}+\frac{\Pi^{i t}(s)}{s-M_{\not{l}}^{2}-\Pi^{i t}(s)} h_{\not}^{*},
\end{aligned}
$$

which, as follows from eq. (5.6), are to be evaluated on the mass shell of the $i$-th quasiparticle [35]. Using eqs. (5.3) and the tree-level relation $H_{i i}=16 \pi M_{i} \Gamma_{i}$, we obtain

$$
\begin{aligned}
& \Pi_{i j}\left(M_{i}^{2}\right)=-i \frac{\operatorname{Re} H_{i \downarrow}}{16 \pi}, \\
& \Pi_{\not h}\left(M_{i}^{2}\right)=M_{\not} \Gamma_{\ell}\left[\frac{1}{\pi}\left(\ln \left(\frac{M_{i}^{2}}{M_{\not h}^{2}}\right)-\frac{M_{i}^{2}-M_{\not{l}}^{2}}{M_{\not \lambda}^{2}}\right)-i\right] .
\end{aligned}
$$


Note that, for $M_{i} \approx M_{i}$, each term in the round brackets in eq. (5.9b) vanishes and therefore this contribution can be neglected. On the other hand, for $M_{i} \ll M_{i}$, the difference in the round brackets of eq. (5.9b) only increases slowly with growing $M_{i} / M_{i}$ and is negligibly small compared to the $M_{i}^{2}-M_{i}^{2}$ term in the denominator of eq. (5.8). Therefore, for practical purposes, it is sufficient to keep only the imaginary part of eq. (5.9b), i.e. use $\Pi_{i k}\left(M_{i}^{2}\right) \approx-i M_{i} \Gamma_{i}$. In this way, we arrive at the effective Yukawa couplings

$$
\mathbf{h}_{i}^{(c)}=h_{i}\left[1-(+) i \frac{H_{i \ell}}{32 \pi}\left(1+\frac{H_{i \downarrow}^{*}}{H_{i \ell}}\right) \frac{1}{\Delta M_{i \not}^{2}+(-) i M_{i} \Gamma_{i}}\right] .
$$

The self-energy contribution to the $C P$-violating parameter in vacuum takes the form

$$
\epsilon_{i}^{\mathrm{vac}} \equiv \frac{\Gamma_{\psi_{i} \rightarrow b b}-\Gamma_{\psi_{i} \rightarrow \bar{b} \bar{b}}}{\Gamma_{\psi_{i} \rightarrow b b}+\Gamma_{\psi_{i} \rightarrow \bar{b} \bar{b}}},
$$

and we find

$$
\epsilon_{i}^{\mathrm{vac}}=\operatorname{Im}\left(\frac{H_{i \not}}{H_{i \not}^{*}}\right) \frac{\left(M_{i}^{2}-M_{\not}^{2}\right) M_{\ell} \Gamma_{\not}}{\left(M_{i}^{2}-M_{\not}^{2}\right)^{2}+\left(M_{\ell} \Gamma_{\ell}\right)^{2}},
$$

cf. eq. (2.29).

Following ref. [11] (see appendix A for a comparison of conventions), the time-derivative of the asymmetry can be written in terms of the effective Yukawa couplings as

$$
\frac{\mathrm{d} \eta}{\mathrm{d} t} \approx \int_{q} \theta\left(q_{0}\right)\left[\mathbf{h}_{i} \mathbf{h}_{j}^{*} G_{\delta}^{0, i j}(t, q)-\mathbf{h}_{i}^{c *} \mathbf{h}_{j}^{c} G_{\delta}^{0, i j}(t, q)\right] \widetilde{\Pi}_{\rho}(q),
$$

where $G_{\delta}^{0, i j}(t, q)$ is the non-equilibrium part of the tree-level propagator. As discussed in section 4 , the tree-level heavy-neutrino propagator may be written in the on-shell approximation (see ref. [11])

$$
G_{\delta}^{0, i j}\left(t, q_{0}>0\right)=2 \pi \delta\left(q_{0}-\bar{\omega}\right) \frac{1}{2 \bar{\omega}} \delta n^{i j}(t, \mathbf{q}) .
$$

The time-derivative of the asymmetry is then found to be

$$
\begin{aligned}
\frac{\mathrm{d} \eta}{\mathrm{d} t} \approx & 2 \sum_{i} \int_{\mathbf{q}} \frac{M_{i}}{\bar{\omega}} \delta n^{i i}(t, \mathbf{q}) \Gamma_{i}(\bar{\omega}, \mathbf{q}) \epsilon_{i}^{\mathrm{vac}}(\bar{\omega}, \mathbf{q}) \\
& +2 \operatorname{Im} H_{12} \int_{\mathbf{q}} \frac{\widetilde{\Pi}_{\rho}(\bar{\omega}, \mathbf{q})}{\bar{\omega}} \operatorname{Im} \delta n^{12}(t, \mathbf{q}) .
\end{aligned}
$$

With the exception of the additional factor of $\Delta M_{12}^{2} R_{12}$, which is of order unity in the weakly-resonant regime, the oscillation contribution resembles that appearing in eq. (3.60). On the other hand, the mixing contribution does not see the modifications that resulted in eq. (3.60) from the interference terms in the final line of eq. (3.59). This conclusion is suggestive that the approach of refs. [9-11], although accounting successfully for both mixing and oscillation, may not fully capture the interference of these two effects. However, as identified earlier in section 3, the approximation used in (appendix A.1 of) ref. [11] does not hold in the weak-washout regime studied here, and it would be of interest to study the impact of these interference effects quantitatively in the strong-washout regime. 


\section{Phenomenological implications}

In order to get a feeling for the relative size of the mixing, oscillation and interference contributions, we now compute the asymmetry for various values of the degeneracy parameter

$$
R \equiv \frac{M_{2}^{2}-M_{1}^{2}}{M_{1} \Gamma_{1}+M_{2} \Gamma_{2}} .
$$

In the weak-washout regime, to which we limit ourselves in this work, the impact of the initial conditions on the final asymmetry is non-negligible. Therefore, in order to ensure that the produced asymmetry is of dynamical origin, we need to choose $C$-symmetric initial conditions in our numerical analysis. As is shown in refs. [72, 94], for a non-degenerate mass spectrum, the Lagrangian in eq. (1.1) is $C$-symmetric if either $\operatorname{Im} H_{12}=0$ or $\operatorname{Re} H_{12}=0$. It is also automatically $C$-symmetric for a degenerate mass spectrum. This can be summarized conveniently by forming the familiar basis-independent measure of both $C$ - and $C P$-violation, the Jarlskog invariant

$$
J=\operatorname{Im} \operatorname{Tr} \boldsymbol{H} \boldsymbol{M}^{3} \boldsymbol{H}^{\top} \boldsymbol{M},
$$

which vanishes when any of the $C$-conserving conditions are satisfied (see appendix B and ref. [72] for more details).

Whereas the r.h.s. of eq. (2.28) vanishes in the limit $\operatorname{Im} H_{12}=0$, it does not automatically vanish when $\operatorname{Re} H_{12}=0$ except in the absence of initial flavour coherences, i.e. for $\delta n^{12}(0)=0$. This implies that there are two ways to specify the initial conditions such that the asymmetry automatically vanishes if the Lagrangian is $C$-conserving. The first possibility, studied in ref. [72], is to choose $\mathcal{K}^{12}=0$, i.e. to set the leading oscillation term to zero. The second possibility is to require that the initial conditions be $C$-symmetric, which, in the Heisenberg picture, corresponds to choosing $G_{\delta}^{i j}(0,0)$ diagonal in the mass eigenbasis (see appendix B for more details). It can be shown by virtue of the constraints provided on the two-point functions by causality, unitarity, CTP invariance and Hermiticity (see ref. [84]) that the source $K^{m n}$ has the same properties as the statistical one-loop self-energy in the thermal bath. Thus, it follows that $\mathcal{K}^{m n}=\mathcal{K}^{n m}$. Using this symmetry and requiring $G_{\delta}^{12}(0,0)$ to vanish, we obtain

$$
\mathcal{K}^{12}=\mathcal{K}^{21}=-\frac{\mathcal{K}^{11} G_{R}^{11}(0) G_{A}^{12}(0)+\mathcal{K}^{22} G_{R}^{12}(0) G_{A}^{22}(0)}{G_{R}^{11}(0) G_{A}^{22}(0)+G_{R}^{12}(0) G_{A}^{12}(0)},
$$

where $0=0^{+}$for the retarded and $0=0^{-}$for the advanced propagator. The properties of the propagators also imply $G_{A}^{i j}(0)=G_{R}^{j i}(0)$, and therefore it is sufficient to consider for example only the retarded one. Expressed in terms of its Wigner transform, the retarded propagator takes the form

$$
G_{R}^{i j}(0)=2 \int_{0}^{\infty} \frac{\mathrm{d} q_{0}}{2 \pi} \operatorname{Re} G_{R}^{i j}\left(q_{0}\right) \approx-2 \operatorname{Im} \frac{1}{\Delta \Omega^{2}} \sum_{k=1}^{2} \frac{(-1)^{k}}{2 \omega_{k}}\left[\operatorname{adj} \boldsymbol{D}_{R}\left(\omega_{k}\right)\right]^{i j},
$$

where we have used eq. (2.14) and Cauchy's theorem to evaluate the integral approximately. It follows from this expression that $G_{R}^{12} \propto \Pi_{R}^{12} \propto \operatorname{Re} H_{12}$ and similarly $G_{A}^{12} \propto \Pi_{A}^{12} \propto \operatorname{Re} H_{12}$. 
Thus, we find $\mathcal{K}^{12}=\mathcal{K}^{21} \propto \operatorname{Re} H_{12}$, such that, for a $C$-symmetric choice of the initial conditions, the produced asymmetry automatically vanishes when either $\operatorname{Im} H_{12}=0$ or $\operatorname{Re} H_{12}=0$. In other words, although this is not immediately obvious because the $\psi_{i}$ are not necessarily on shell, for a $C$-symmetric choice of the initial conditions, the mixing and oscillation sources of the asymmetry are proportional to the Jarlskog invariant in eq. (6.2) (see ref. [72] for a detailed discussion). As has been shown in refs. [9-11], in the strongwashout regime, in which the final asymmetry is known to be independent of the initial conditions, the solution of the kinetic equations automatically possesses this property. For the toy model under consideration, we demonstrate this in appendix $\mathrm{C}$.

For the numerical analysis, it is more convenient to evaluate eq. (2.2) directly. Substituting the Wigner transform of eq. (2.9) into eq. (2.2) and neglecting the sub-leading off-shell contributions, the time-derivative of the asymmetry takes the form

$$
\begin{aligned}
\frac{\mathrm{d} \eta}{\mathrm{d} t}= & 4 \operatorname{Im} H_{12} \int_{\mathbf{q}} \frac{\epsilon_{a b}}{\left|\Delta \Omega^{2}\right|^{2}} \widetilde{\Pi}_{\rho}\left(\left(\omega_{a}+\omega_{b}\right) / 2, \mathbf{q}\right) \\
& \times \operatorname{Im}\left[\frac{\left[\operatorname{adj} \boldsymbol{D}_{R}\left(\omega_{a}, \mathbf{q}\right)\right]^{1 m}}{2 \Omega_{a}} \mathcal{K}^{m n}(\mathbf{q}) \frac{\left[\operatorname{adj} \boldsymbol{D}_{A}\left(\omega_{b}, \mathbf{q}\right)\right]^{n 2}}{2 \Omega_{b}^{*}} e^{-i\left(\Omega_{a}-\Omega_{b}^{*}\right) t}\right],
\end{aligned}
$$

where we have restored the common momentum $\mathbf{q}$ and used the same notational conventions as in eq. (3.57). We emphasize that the numerical analysis of the present section is performed for the full solution, without an expansion to a given order in $\Pi / \Delta M^{2}$. Were we to expand eq. (6.5) in powers of $\Pi_{R(A)}$, we would recover eq. (2.28). We would also like to emphasize that the leading order expansion (eq. (2.28)) provides a very accurate approximation to eq. (6.5), as has already been pointed out in ref. [72]. At the initial time surface $t=0$, the Heisenberg- (eq. (2.19)) and interaction-picture (eq. (3.59)) propagators coincide. This can be used to extract from eq. (6.5) the mixing, oscillation, and interference sources, as identified in eq. (3.59).

For the numerical examples, following ref. [72], we choose $T=\mu$ and $M_{1}=\mu$, where $\mu$ is the $\overline{\mathrm{MS}}$ renormalization scale. The second mass parameter $M_{2}$ can be expressed in terms of the degeneracy parameter $R$ in eq. (6.1). In figure 1, we plot the three contributions to the final asymmetry, as well as the total asymmetry itself, for four choices of initial conditions and with would-be Yukawa couplings $h_{1}=0.5 \mu \exp (-i)$ and $h_{2}=-0.8 \mu \exp (-2 i / 3)$. The same is plotted in figure 2 for larger "Yukawa" couplings $h_{1}=\mu \exp (-i)$ and $h_{2}=$ $-1.6 \mu \exp (-2 i / 3)$. The red, dashed line indicates the contribution from the mixing source; the green, dotted line the contribution from the oscillation source; the blue, dash-dotted line the contribution from the interference terms; and the solid, black line the total asymmetry. By comparing figures 1 and 2, we see that the relative size of the various contributions is unaffected by the change in the "Yukawa" couplings. This can be understood in terms of their common parametric dependence on the couplings and mass splittings, as identified in section 3. A more significant effect is seen only for $R>1$ in the case of $\mathcal{K}^{12} \neq 0$ due to the additional dependence on the "Yukawa" couplings introduced via eq. (6.3), as is necessary in order to specify $C$-symmetric initial conditions in the weak-washout regime. 

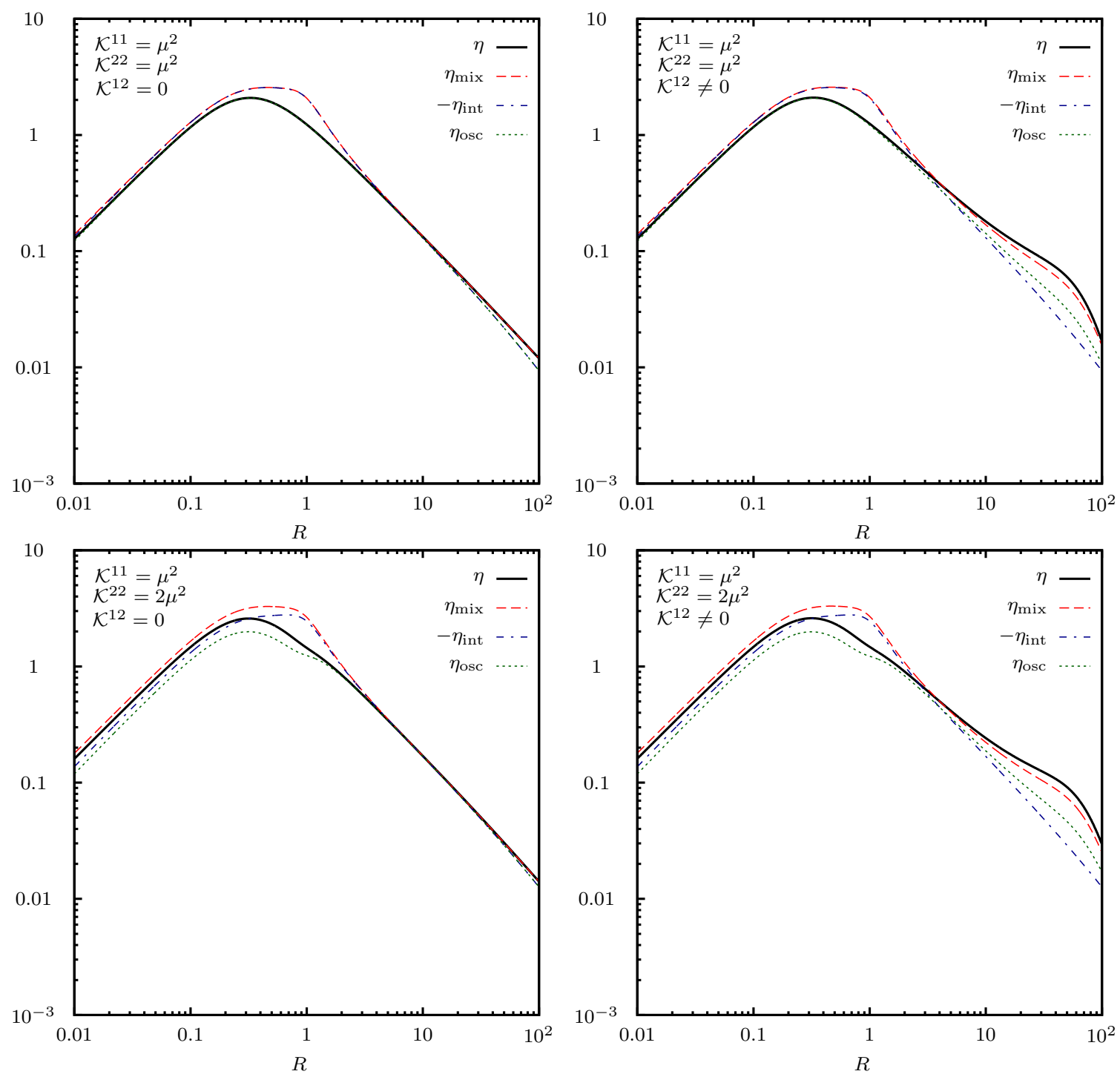

Figure 1. Contributions of the mixing $\left(\eta_{\mathrm{mix}}\right)$, oscillation $\left(\eta_{\mathrm{osc}}\right)$ and interference $\left(\eta_{\text {int }}\right)$ sources to the asymptotic value of the asymmetry as functions of the degeneracy parameter $R$ for various ( $C$-conserving) choices of the initial conditions and with "Yukawa" couplings $h_{1}=0.5 \mu \exp (-i)$ and $h_{2}=-0.8 \mu \exp (-2 i / 3)$.

The numerical results can be interpreted in two ways:

(i) On the one hand, taking the Boltzmann approximation (effective "Yukawa" couplings but diagonal number densities) as the "benchmark", one would think of the sum of the oscillation (green, dotted lines) and interference contributions (blue, dash-dotted lines) as the correction to the original approximation. With this interpretation in mind, we plot in figure 3 the total asymmetry (solid, black line) versus the mixing contribution (red, dashed line) and the sum of the oscillation and interference terms (orange, dash-dotted line). In agreement with expectations, the correction from the oscillation and interference terms is large for $R \sim 1$ and is very small for hierarchical 

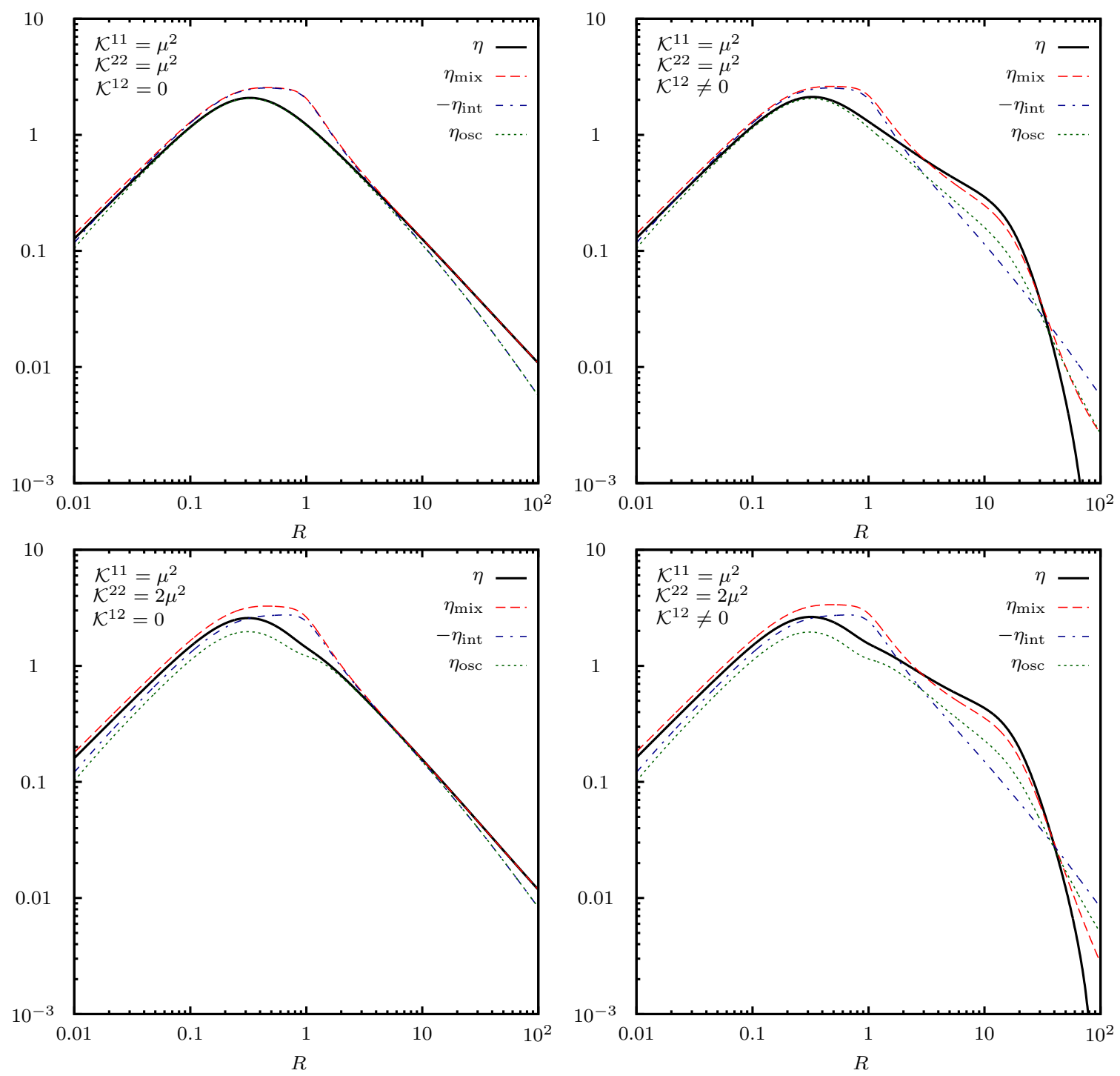

Figure 2. Contributions of the mixing $\left(\eta_{\mathrm{mix}}\right)$, oscillation $\left(\eta_{\mathrm{osc}}\right)$ and interference $\left(\eta_{\text {int }}\right)$ sources to the asymptotic value of the asymmetry as functions of the degeneracy parameter $R$ for various ( $C$-conserving) choices of the initial conditions and with "Yukawa" couplings $h_{1}=\mu \exp (-i)$ and $h_{2}=-1.6 \mu \exp (-2 i / 3)$. Note that the deviation between the black and coloured curves at $R \sim 100$ is not related to the deviations in figure 5 .

mass spectra. Interestingly, it is also small for quasi-degenerate mass spectra. All in all, we find that the Boltzmann approximation, which is equivalent to the mixing contribution (red, dashed line), agrees well with the total asymmetry, with the exception of the region $R \sim 1$.

(ii) Instead, taking the density matrix approximation (tree-level "Yukawa" couplings but off-diagonal number densities) as the "benchmark", one would think of the sum of the mixing (red, dashed lines) and the interference terms (blue, dash-dotted lines) as the correction to the original approximation. With this interpretation in mind, we 

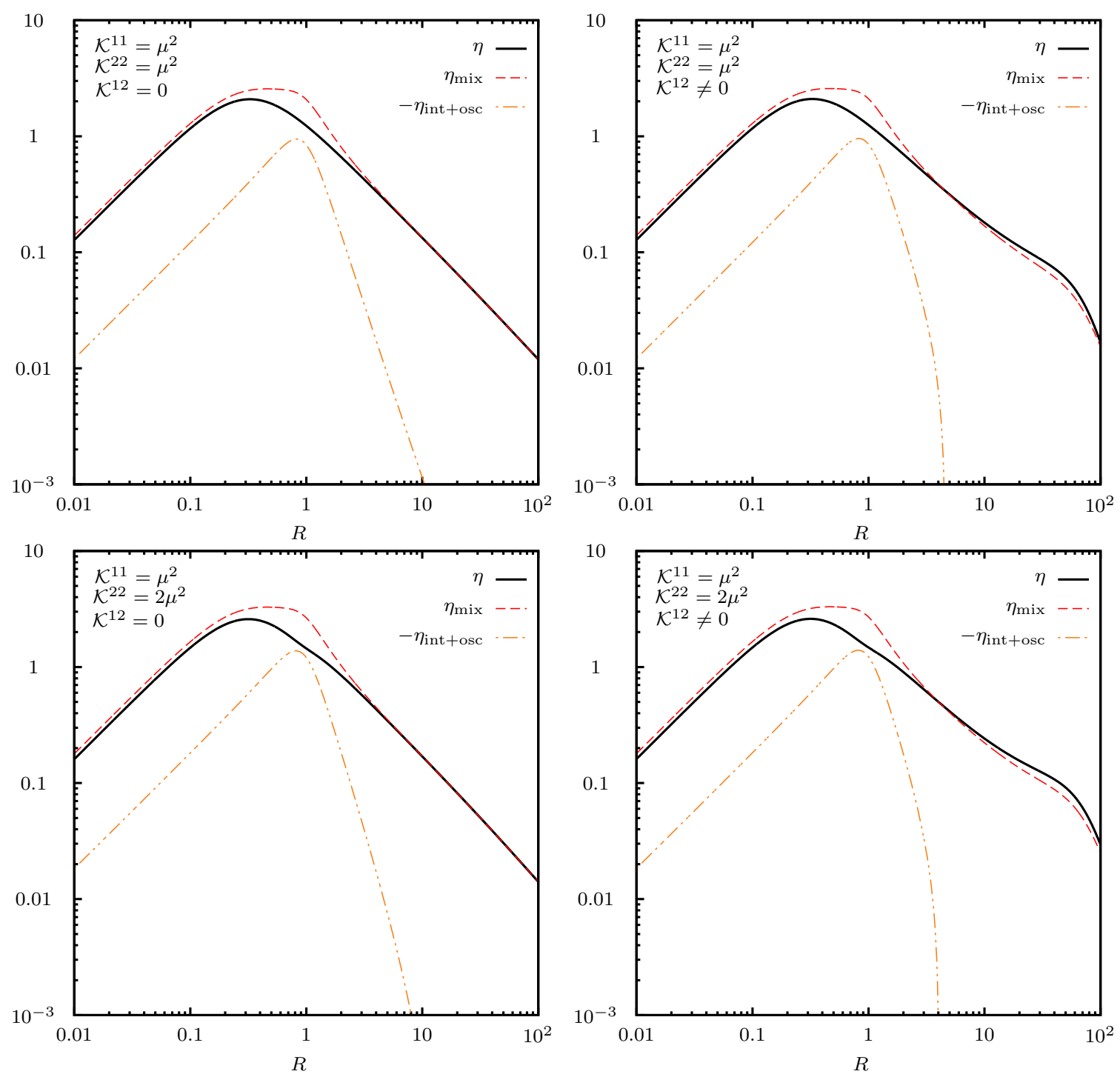

Figure 3. Numerical results interpreted in terms of the Boltzmann "benchmark" $\left(\eta_{\operatorname{mix}}\right)$ plus corrections $\left(\eta_{\text {osc }+ \text { int }}\right)$ as functions of the degeneracy parameter $R$ for various $(C$-conserving) choices of the initial conditions and with "Yukawa" couplings $h_{1}=0.5 \mu \exp (-i)$ and $h_{2}=-0.8 \mu \exp (-2 i / 3)$.

plot in figure 4 the total asymmetry (solid, black line) versus the oscillation contribution (green, dotted line) and the sum of the mixing and interference terms (brown, dash-dotted line). In this case, we see that the density matrix approximation, which is equivalent to the oscillation contribution (green, dotted line), agrees well with the total asymmetry when the number densities of the two flavours are of similar magnitudes (upper two panels of figure 4). On the other hand, when the number densities of the two flavours are not similar (lower two panels of figure 4), as is enforced in the weak-washout regime by choosing differing initial conditions for the two flavours, we see that the density matrix approximation underestimates the total asymmetry for smaller $R$. This observation can be understood from the analytic results given 

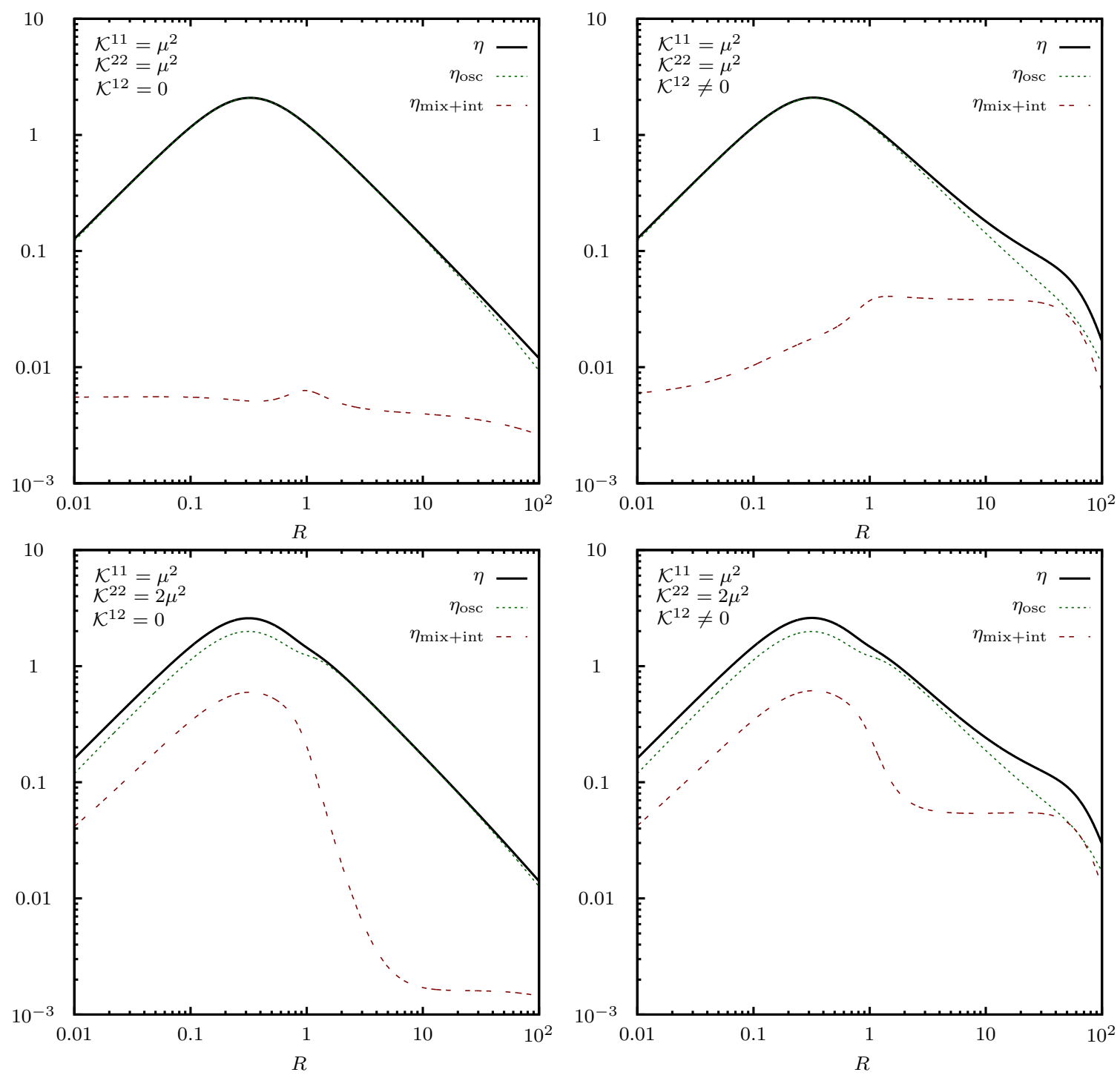

Figure 4. Numerical results interpreted in terms of the density matrix "benchmark" $\left(\eta_{\mathrm{osc}}\right)$ plus corrections $\left(\eta_{\text {mix }}+\right.$ int $)$ as functions of the degeneracy parameter $R$ for various $(C$-conserving) choices of the initial conditions and with "Yukawa" couplings $h_{1}=0.5 \mu \exp (-i)$ and $h_{2}=-0.8 \mu \exp (-2 i / 3)$.

in eqs. (3.63) and (3.64) for the effective $C P$-violating parameter. Specifically, with the density matrix approximation as the benchmark, the interference terms can be seen as a modification to the mixing source. This modification introduces a relative sign between the contribution to the asymmetry from the two flavours. Hence, when the deviations from equilibrium of the two flavours are similar, the mixing contribution is strongly suppressed. On the other hand, when this is not the case, the cancellation is no longer exact and both the oscillation and mixing sources contribute additively to the asymmetry, leading to an underestimate if one were to neglect one or other of these sources. In spite of the possibility that the interference terms may not be captured fully in the analysis of refs. [9-11], we nevertheless see as marked 
an enhancement in the present analysis up to a maximal factor of two for certain values of the parameters. It remains to be seen the extent to which the interference terms modify the final asymmetry for more-realistic phenomenological models in the strong-washout regime and for an expanding background. However, since the mixing, oscillation and interference terms all share common parametric dependence upon the "Yukawa" couplings and mass splittings, one can reasonably anticipate that all three effects may be of relevance for such models in the weakly-resonant (or overlapping) regime $\Gamma_{i} \ll \Delta M \ll \bar{M}$. We would like to emphasize that, for large $R$, the oscillation source in figure 4 cannot be directly associated with the results of section 4 , because the latter are only applicable for $\omega_{2} \simeq \omega_{1}$, which is only fulfilled for quasidegenerate mass spectra. This point is illustrated in figure 5 , where we compare the total asymmetry computed taking into account the full shell structure to the total asymmetry and the oscillation contribution computed using the middle-shell approximation $\omega_{1}=\omega_{2}=\omega$ in eq. (6.5). As expected, for hierarchical mass spectra the results differ by orders of magnitude.

For completeness, we also plot in figure 6 the same comparisons for the larger choice of "Yukawa" couplings and for the initial condition $\mathcal{K}^{12} \neq 0$. We draw attention to the bottom right panel of this figure, where the underestimate of the lepton asymmetry provided by the oscillation source alone is very apparent. The maximum factor of two enhancement in the asymmetry can also be seen clearly when both rather than only one (oscillation or mixing) source are accounted for.

\section{Conclusions and outlook}

For a hierarchical mass spectrum of heavy Majorana neutrinos, baryogenesis via leptogenesis can be studied in detail using conventional flavour-diagonal Boltzmann equations. On the other hand, for a mildly quasi-degenerate mass spectrum, the Boltzmann approximation is insufficient, and there is ongoing work on the first-principles derivation of kineticand systematically-improved Boltzmann equations capable of fully accounting for all relevant effects, in particular the resonant enhancement of $C P$-violating parameters and the oscillation between different flavours. In practice, it is necessary to find consistent approximation schemes in order to render the solution of these equations tractable for the purpose of performing numerical scans of the available parameter space.

The mixing of particle flavours and the oscillations between them are two physically distinct and identifiable phenomena, as is known from the neutral $K, D, B$ and $B_{s}$ systems [1]. In this work, using Kadanoff-Baym equations, we confirm (in the weak-washout regime) that mixing and oscillations indeed provide two distinct sources of lepton asymmetry, which can be readily identified by means of the shell structure of the resummed heavy-neutrino propagators. The mixing contributions correspond to the usual $\mathrm{CP}$-violation in decay and live on the mass shell of the corresponding quasi-particles with energy $\omega_{i}$. Instead, flavour oscillations between the heavy neutrinos and interference between mixing and oscillation can be identified with an "oscillation shell" of energy $\bar{\omega}=\left(\omega_{1}+\omega_{2}\right) / 2$. 

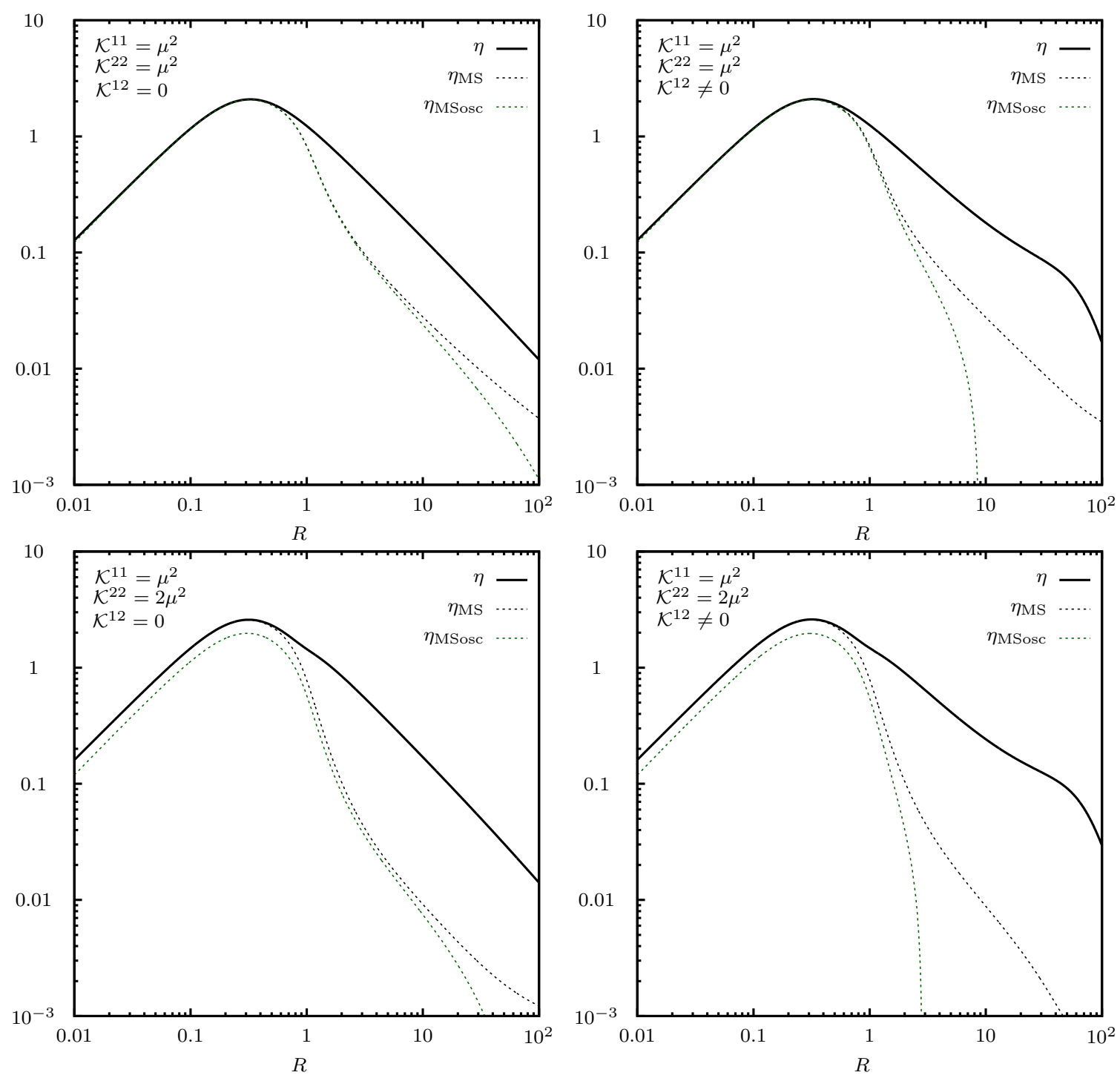

Figure 5. Numerical results for the total asymmetry computed from eq. (6.5) using the complete shell structure (solid black line), and the total asymmetry and the oscillation contribution (dotted black green lines respectively) for the middle-shell approximation $\omega_{1}=\omega_{2}=\bar{\omega}$ as functions of the degeneracy parameter $R$.

Historically, leptogenesis was first studied in the Boltzmann approximation, i.e. using diagonal number densities with the transition amplitudes computed in vacuum. From the perspective of this approximation, one would think of the sum of the oscillation and interference contributions as the correction. In agreement with expectations, this correction is large when the difference of the masses is comparable to the decay widths and is very small for hierarchical mass spectra. Interestingly, it is also small for quasi-degenerate mass spectra. Within the past decade, a lot of work has been devoted to the re-analysis of resonant leptogenesis within the density matrix formalism, i.e. taking into account offdiagonals of the matrix of number densities. From this perspective, one would instead 

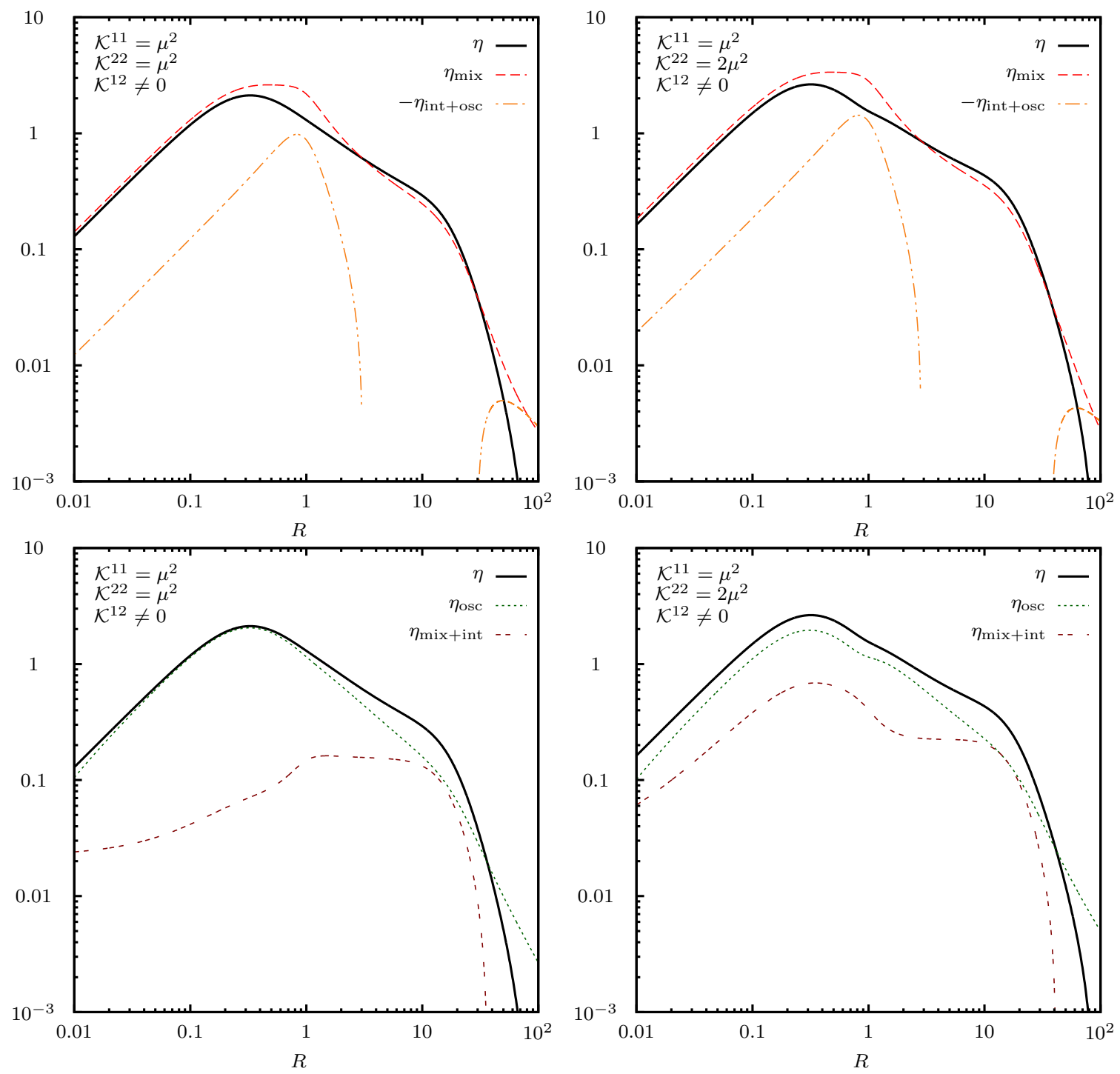

Figure 6. Numerical results interpreted in terms of the Boltzmann $\left(\eta_{\operatorname{mix}}\right)$ and density matrix $\left(\eta_{\mathrm{osc}}\right)$ "benchmarks" as functions of the degeneracy parameter $R$ for various ( $C$-conserving) choices of the initial conditions and with "Yukawa" couplings $h_{1}=\mu \exp (-i)$ and $h_{2}=-1.6 \mu \exp (-2 i / 3)$. The oscillation contribution (dotted green line) is in excess of the total asymmetry (solid black line) for large $R$ due to a change of sign in the mixing plus interference terms, which is not visible on the plot.

think of the sum of the mixing and interference terms as the correction. When the number densities of the two flavours are of similar size, this correction is small. On the other hand, when the number densities of the two flavours are not similar, the correction becomes sizable even for quasi-degenerate mass spectra, i.e. in the parameter range where it originally was thought to be small. We find that the mixing and oscillation sources are of the same sign, contributing additively to the final asymmetry up to a factor of two, in agreement with the conclusions of refs. [9-11]. However, we also find that the interference terms may lead to a suppression of the contribution from mixing. Hence, it would be of interest to perform 
an equivalent analysis for a realistic phenomenological model in the strong-washout regime and for an expanding background.

By comparing the Heisenberg- and interaction-picture Kadanoff-Baym equations, we have found identical results, illustrating the self-consistency and complementarity of these two significantly different approaches. We note that this exact agreement relied upon the interference between the mixing and oscillation contributions in the transport equations for the number densities. Whereas the Heisenberg-picture Kadanoff-Baym equations are very useful for studying features of the regimes that cannot be addressed by either Boltzmann (not applicable for quasi-degenerate mass spectra) or density matrix (not applicable for hierarchical mass spectra) approaches, their use in phenomenological studies is severely limited by the difficulty of solving them. The fact that the interaction-picture KadanoffBaym equations, which are much easier to solve, are identical to the Heisenberg-picture ones means that we now have a convenient tool that allows us to treat hierarchical and quasi-degenerate mass spectra on an equal footing, thereby performing parameter scans over a large range.

\section{Acknowledgments}

P.M. would like to express a debt of gratitude to Bhupal Dev, Marco Drewes, Björn Garbrecht, Apostolos Pilaftsis and Daniele Teresi for many fruitful, enjoyable and illuminating discussions in this area. The authors would like to thank Björn Garbrecht and Daniele Teresi for careful reading of the manuscript and helpful comments and suggestions. The work of P.M. was supported in part by a University Foundation Fellowship (TUFF) from the Technische Universität München and the Science and Technologies Facilities Council (STFC) under Grant No. ST/L000393/1. A.K. and H.V. acknowledge financial support by the European Union under Grant No. PITN-GA-2011-289442 (FP7 Initial Training Network "Invisibles"). All three authors acknowledge support from the Deutsche Forschungsgemeinschaft (DFG) under Grant No. EXC-153 (Excellence Cluster "Universe").

\section{A Non-equilibrium field theory}

The purpose of this appendix is two fold: firstly, it is intended to provide a brief outline of the background to the Heisenberg- and interaction-picture realizations of the KadanoffBaym approach to transport phenomena; secondly, it serves as a collection of the definitions and notational conventions of the various two-point functions and self-energies that appear in the body of this manuscript. In addition and in order to aid the comparison of this work with the existing literature, we identify the correspondence of the conventions employed herein with those appearing elsewhere.

In the study of transport phenomena, we are interested in the statistical or ensemble expectation values (EEVs) of operators evaluated at a given time. Specifically, in the Schrödinger picture, these EEVs take the generic form

$$
\langle\bullet\rangle(t) \equiv \mathcal{Z}^{-1} \operatorname{tr} \rho(t) \bullet,
$$


where $\rho(t)$ is the quantum-statistical density operator and $\mathcal{Z}=\operatorname{tr} \rho$ is the partition function. This is in stark contrast to scattering-matrix theory, where we are instead interested in the overlap of states evaluated at different times: specifically, in and out asymptotic states. This in-out formalism naturally lends itself to a path-integral description, leading to a time integral that runs from the infinitely-distant past to the infinitely-distant future. On the other hand, EEVs contain the overlap of states evaluated at the same time, i.e. two in (or two out) states.

Closed-time path. In order to define a path-integral representation of EEVs, we must deform a contour in the complex-time plane that takes us from the in state to the out state and back again. This construction gives rise to the so-called in-in or closed-time path (CTP) formalism due to Schwinger and Keldysh [79, 80].

The CTP contour comprises two branches: one running forwards in time, which we refer to as the time-ordered branch, and one running backwards in time, which we refer to as the anti-time-ordered branch. On this contour, we may introduce a path-ordering operator $\mathrm{T}_{\mathcal{C}}$. Given two field operators with times $x^{0}$ and $y^{0}$ both lying on the timeordered branch, the path ordering reduces to the usual time ordering. When both times lie instead on the anti-time-ordered branch, the path ordering corresponds to anti-time ordering. Finally, times lying on the time-ordered branch are, for the purposes of path ordering, always 'earlier' than those on the anti-time-ordered branch. As a consequence of our ability to place field operators on either of the two branches, the CTP formalism leads to a doubling of degrees of freedom. The need for the latter can be understood as follows: we need sufficient degrees of freedom to build both the statistical ensemble and excitations within it.

In the same way that expectation values of operators can be written in any one of the three equivalent pictures of quantum mechanics, viz. the Schrödinger, interaction (Dirac) and Heisenberg pictures, so too can the corresponding operator-level representation of the CTP formalism. These three pictures are coincident at a boundary time $\tilde{t}_{i}$, i.e.

$$
\rho_{H}\left(; \tilde{t}_{i}\right)=\rho_{I}\left(\tilde{t}_{i} ; \tilde{t}_{i}\right)=\rho_{S}\left(\tilde{t}_{i} ; \tilde{t}_{i}\right),
$$

where we have indicated Heisenberg-, interaction- (Dirac-) and Schrödinger-picture operators by subscripts $H, I$ and $S$, respectively. In the Heisenberg-picture, field operators are time-dependent, evolving with the full Hamiltonian $H_{H}\left(\tilde{t} ; \tilde{t}_{i}\right)$, whereas the density operator is time-independent, encoding the initial conditions at the time $\tilde{t}_{i}$. Here, following ref. [84], we have indicated the implicit dependence on the boundary time $\tilde{t}_{i}$ by means of a semicolon. On the other hand, in the interaction picture, both the field and density operators are time-dependent, evolving respectively under the influence of the free and interaction parts of the Hamiltonian $H_{I}^{0}\left(; \tilde{t}_{i}\right)$ and $H_{I}^{\text {int }}\left(\tilde{t} ; \tilde{t}_{i}\right)$.

Pinch singularities. It is well-known that perturbation theory breaks down in the Heisenberg-picture realization of non-equilibrium field theory as a result of so-called pinch singularities or secular terms. At the level of the perturbation series, this pathological behaviour arises from ill-defined products of Dirac delta functions with identical arguments. 
Their origin can be understood in terms of the Fermi golden rule: for systems in which time-translational invariance is broken, the relevant expansion parameter is the product of the coupling, $g$ say, and the time $t$ over which the interactions have been permitted to take place. Thus, for $t>1 / g$, the perturbation series will not converge. As an example, we may consider the exponential approach to equilibrium governed by a decay rate $\Gamma \propto g$ : an expansion of $e^{-\Gamma t}$ in powers of the coupling exists only for $t<1 / g$. In the CTP formalism, the time over which the interactions have been permitted to take place corresponds to the length of the CTP contour. In the Heisenberg-picture realization, this contour is extended to infinity, thereby leading to the emergence of pinch singularities out of equilibrium. On the other hand, it has been shown in ref. [84] that the CTP contour is necessarily of finite length in the interaction picture and, as a result, a well-defined perturbation theory does indeed exist. The contour is bounded from the left by the initial (boundary) time $\tilde{t}_{i}$ and from the right by the final time $\tilde{t}$, at which the EEV is calculated. One is then led to introduce the concept of a macroscopic time $t=\tilde{t}-\tilde{t}_{i}$, where the tilde notation, which we have hitherto not qualified, is reserved for the microscopic times of the operators.

Ensemble expectation value. In the Heisenberg picture, the ensemble expectation value $(\mathrm{EEV})$ is written in bra-ket notation as:

$$
\langle\bullet\rangle_{0}=\operatorname{tr} \rho_{H}\left(; \tilde{t}_{i}\right) \bullet,
$$

where the Heisenberg-picture density operator $\rho_{H}\left(; \tilde{t}_{i}\right)$ is evaluated at the macroscopic time $t=\tilde{t}_{i}-\tilde{t}_{i}=0$. In the interaction picture, the EEV is written as

$$
\langle\bullet\rangle_{t}=\operatorname{tr} \rho_{I}\left(\tilde{t} ; \tilde{t}_{i}\right) \bullet,
$$

where the interaction-picture density operator $\rho_{I}\left(\tilde{t} ; \tilde{t}_{i}\right)$ is instead evaluated at the macroscopic time $t=\tilde{t}-\tilde{t}_{i} \neq 0$. Hereafter, we suppress the dependence of both Heisenberg- and interaction-picture operators, as well as all two-point functions and self-energies, on the boundary time $\tilde{t}_{i}$.

Coordinate conventions. The coordinate-, Wigner- and double-momentum-space representations of the various two-point functions are, following ref. [84], distinguished only by the form of their arguments. Interaction-picture two-point functions are distinguished from their Heisenberg-picture counterparts by a superscript 0 , in the case of the tree-level two-point functions, and explicit dependence on the microscopic time $\tilde{t}$, in the case of selfenergies and the resummed two-point functions. Wherever possible, coordinate-space variables are denoted by the lower-case Roman characters $x, y, \ldots$; and their Fourier-conjugates by the four-momenta $p, p^{\prime}, \ldots$. The central and relative coordinates are denoted by the upper-case Roman characters $X$ and $R$, respectively, where

$$
R^{\mu}=x^{\mu}-y^{\mu}, \quad X^{\mu}=\left(x^{\mu}+y^{\mu}\right) / 2 .
$$

Finally, the characters $q$ and $Q$ are reserved for the central and relative momenta

$$
q^{\mu}=\left(p^{\mu}+p^{\mu \prime}\right) / 2, \quad Q^{\mu}=p^{\mu}-p^{\mu \prime} .
$$

These conventions are summarized in table 1. 


\begin{tabular}{|l|c|c|}
\hline & Heisenberg picture & Interaction picture \\
\hline Coordinate space & $(x, y)$ & $(x, y, \tilde{t})$ \\
Wigner space & $(q, X)$ & $(q, X, \tilde{t})$ \\
Double-momentum space & $\left(p, p^{\prime}\right)$ & $\left(p, p^{\prime}, \tilde{t}\right)$ \\
\hline
\end{tabular}

Table 1. The form of the arguments of the various resummed two-point functions and self-energies, indicating whether they belong to the Heisenberg or interaction picture and if they are expressed in the coordinate-, Wigner- or double-momentum-space representation. The interaction picture is consistently identified by the appearance of an explicit dependence on the microscopic time $\tilde{t}$.

Double Fourier and Wigner transforms. The double Fourier transform $f\left(p, p^{\prime}\right)$ of a function $f(x, y)$ is defined as follows:

$$
\begin{aligned}
f\left(p, p^{\prime}\right) & \equiv \mathcal{F}_{y}\left[\mathcal{F}_{x}[f(x, y)](p)\right]\left(-p^{\prime}\right) \equiv \mathcal{F}_{x}\left[\mathcal{F}_{y}[f(x, y)]\left(-p^{\prime}\right)\right](p) \\
& \equiv \int_{-\infty}^{+\infty} \mathrm{d}^{4} x \int_{-\infty}^{+\infty} \mathrm{d}^{4} y e^{-i p \cdot x} e^{i p^{\prime} \cdot y} f(x, y) .
\end{aligned}
$$

We emphasize the relative sign in the exponent of the right-most $y$-dependent kernel. This is chosen such that translational invariance $f(x, y)=f(x-y)$ corresponds to the conservation of four-momentum $p=p^{\prime}$.

The Wigner transform $f(q, X)$ of a function $f(x, y)$ is defined as follows:

$$
f(q, X) \equiv \mathcal{F}_{R}[f(x, y)](q) \equiv \int_{-\infty}^{+\infty} \mathrm{d}^{4} R e^{i q \cdot R} f(x, y) .
$$

It may also be written in terms of an inverse transform of the double-momentum representation $f\left(p, p^{\prime}\right)$ :

$$
f(q, X) \equiv \mathcal{F}_{Q}^{-1}\left[f\left(p, p^{\prime}\right)\right](X) \equiv \int_{-\infty}^{+\infty} \frac{\mathrm{d}^{4} Q}{(2 \pi)^{4}} e^{-i Q \cdot X} f\left(p, p^{\prime}\right) .
$$

CTP propagators. In the present discussion of the relevant two-point functions, we denote by the upper-case Roman character $G$ the conventions of ref. [72] and those used throughout the body of this article. Those denoted by the upper-case Greek character $\Delta$ follow the conventions of refs. $[11,84]$. Parenthesized names correspond to the nomenclature of refs. [11, 84] and are placed in the text immediately following the corresponding nomenclature of ref. [72]. Table 2 provides a summary of the relation between these conventions.

The CTP propagator of the would-be heavy neutrinos of the model in eq. (1.1) is defined as

$$
G_{\mathcal{C}}^{[0, i j i}(x, y[\tilde{t}]) \equiv\left\langle\mathrm{T}_{\mathcal{C}}\left[\psi_{H[I]}^{i}(x) \psi_{H[I]}^{j}(y)\right]\right\rangle_{0[t]} .
$$

Objects appearing in brackets ([]) correspond to the interaction-picture definitions. For times $x^{0}$ and $y^{0}$ on the time-ordered branch, $G_{\mathcal{C}}^{[0,] i j}(x, y[, \tilde{t}])$ is equal to the time-ordered (Feynman) propagator $G_{\mathrm{T}}^{[0] i j}(x, y[, \tilde{t}])$. For times $x^{0}$ and $y^{0}$ on the anti-time-ordered branch, $G_{\mathcal{C}}^{[0,] i j}(x, y[, \tilde{t}])$ is equal to the anti-time-ordered (Dyson) propagator $G_{\bar{T}}^{[0, j i j}(x, y[, \tilde{t}])$. When 


\begin{tabular}{|c|c|}
\hline Statistical/Hadamard & $G_{F}^{i j} \equiv \frac{1}{2} i \Delta_{1}^{i j}$ \\
\hline Spectral/Pauli-Jordan & $G_{\rho}^{i j} \equiv-\Delta^{i j}$ \\
\hline Retarded (Advanced) & $G_{R(A)}^{i j} \equiv-\Delta_{R(A)}^{i j}$ \\
\hline Wightman & $G_{\gtrless}^{i j} \equiv i \Delta_{\gtrless}^{i j}$ \\
\hline Hermitian (Principal-part) & $G_{h}^{i j} \equiv-\Delta_{\mathcal{P}}^{i j}$ \\
\hline Self-energies & $\begin{array}{c}\Pi_{\gtrless}^{i j} \longleftrightarrow i \Pi_{\gtrless}^{i j} \\
\Pi_{F}^{i j} \longleftrightarrow \frac{1}{2} i \Pi_{1}^{i j} \\
\Pi_{R(A)}^{i j} \longleftrightarrow-\Pi_{R(A)}^{i j}\end{array}$ \\
\hline
\end{tabular}

Table 2. Comparison of the notations for the various two-point functions and self-energies used in ref. [72] (1.h.s.) versus refs. [11, 84] (r.h.s.).

$x^{0}$ is on the time-ordered branch and $y^{0}$ is on the anti-time-ordered branch, $G_{\mathcal{C}}^{[0,] i j}(x, y[, \tilde{t}])$ is equal to the negative-frequency Wightman propagator $G_{<}^{[0,] i j}(x, y[, \tilde{t}])$. On the other hand, when $x^{0}$ is on the anti-time-ordered branch and $y^{0}$ is on the time-ordered branch, $G_{\mathcal{C}}^{[0,] i j}(x, y[, \tilde{t}])$ is equal to the positive-frequency Wightman propagator $G_{>}^{[0, i i j}(x, y[, \tilde{t}])$. Of the four aforementioned propagators, only two are independent.

Rather than working in terms of path ordering, we may also represent the doubling of degrees of freedom by means of a covariant $\mathbb{S O}(1,1)$ notation [81-83], with the CTP propagator transforming as a rank-2 tensor. However, in order to avoid proliferation of sub- and superscripts, we do not employ this notation in this article.

(Anti)commutator functions. The spectral (Pauli-Jordan) function and the statistical (Hadamard) propagator are defined as follows:

$$
\begin{aligned}
& G_{\rho}^{[0,] i j}(x, y[\tilde{t}])=i\left\langle\left[\psi_{H[I]}^{i}(x), \psi_{H[I]}^{j}(y)\right]\right\rangle_{0[t]} \equiv-\Delta^{[0,] i j}(x, y), \\
& G_{F}^{[0,] i j}(x, y[, \tilde{t}])=\frac{1}{2}\left\langle\left\{\psi_{H[I]}^{i}(x), \psi_{H[I]}^{j}(y)\right\}\right\rangle_{0[t]} \equiv \frac{1}{2} i \Delta_{1}^{[0,] i j}(x, y[\tilde{t}]) .
\end{aligned}
$$

The subscript $F$, indicating the statistical (Hadamard) propagator, should not be confused with the same subscript used in $[11,84]$ to indicate the time-ordered (Feynman) propagator.

We draw attention to the fact that the Wigner transform of the spectral (Pauli-Jordan) function $G_{\rho}(p, X)$ differs from the object $\widetilde{G}(p, X)$ appearing in ref. [103] by an overall factor of $i$. It is for this reason that we have chosen to identify the Wigner representation only by the form of the arguments. Specifically, we have

$$
G_{\rho}^{i j}(q, X[\tilde{t}])=\mathcal{F}_{R}\left[G_{\rho}^{i j}(x, y[, \tilde{t}])\right](q) \equiv i \widetilde{G}_{\rho}^{i j}(q, X[\tilde{t}])
$$

Causal functions. The retarded and advanced propagators are defined in terms of the spectral (Pauli-Jordan) function as follows:

$$
\begin{aligned}
& G_{R}^{i j}(x, y[, \tilde{t}])=\theta\left(x^{0}-y^{0}\right) G_{\rho}^{i j}(x, y[\tilde{t}]) \equiv-\Delta_{R}^{i j}(x, y[\tilde{t}]), \\
& G_{A}^{i j}(x, y[\tilde{t}])=-\theta\left(y^{0}-x^{0}\right) G_{\rho}^{i j}(x, y[\tilde{t}]) \equiv-\Delta_{A}^{i j}(x, y[\tilde{t}]),
\end{aligned}
$$


from which we may obtain the identity

$$
G_{\rho}^{i j}(x, y[, \tilde{t}])=G_{R}^{i j}(x, y[\tilde{t}])-G_{A}^{i j}(x, y[, \tilde{t}]) .
$$

In addition, the Hermitian (principal-part) propagator is defined via

$$
\begin{aligned}
G_{h}^{i j}(x, y[\tilde{t}]) & =\frac{1}{2}\left(G_{R}^{i j}(x, y[\tilde{t}])+G_{A}^{i j}(x, y[, \tilde{t}])\right) \\
& =\frac{1}{2} \operatorname{sign}\left(x^{0}-y^{0}\right) G_{\rho}^{i j}(x, y[\tilde{t}]) \equiv-\Delta_{\mathcal{P}}^{i j}(x, y[, \tilde{t}]) .
\end{aligned}
$$

Note that the superscript 0 does not appear in the interaction-picture cases, since the above identities hold at any order in perturbation theory.

Wightman propagators. The absolutely-ordered Wightman propagators are defined as follows:

$$
\begin{aligned}
G_{>}^{[0,] i j}(x, y[\tilde{t}]) & =\left\langle\psi_{H[I]}^{i}(x) \psi_{H[I]}^{j}(y)\right\rangle \equiv i \Delta_{>}^{[0,] i j}(x, y[, \tilde{t}]), \\
G_{<}^{[0,] i j}(x, y[\tilde{t}]) & =\left\langle\psi_{H[I]}^{j}(y) \psi_{H[I]}^{i}(x)\right\rangle \equiv i \Delta_{<}^{[0,] i j}(x, y[, \tilde{t}]) .
\end{aligned}
$$

These may also be written in terms of the spectral (Pauli-Jordan) function and statistical (Hadamard) propagator:

$$
G_{\gtrless}^{i j}(x, y[, \tilde{t}])=G_{F}^{i j}(x, y[, \tilde{t}]) \mp \frac{i}{2} G_{\rho}^{i j}(x, y[, \tilde{t}]),
$$

yielding the identities

$$
\begin{aligned}
& G_{\rho}^{i j}(x, y[, \tilde{t}])=i G_{>}^{i j}(x, y[, \tilde{t}])-i G_{<}^{i j}(x, y[, \tilde{t}]) \\
& G_{F}^{i j}(x, y[, \tilde{t}])=\frac{1}{2}\left(G_{>}^{i j}(x, y[, \tilde{t}])+G_{<}^{i j}(x, y[, \tilde{t}])\right) .
\end{aligned}
$$

Time-ordered propagators. The time-ordered (Feynman) and anti-time-ordered (Dyson) propagators do not feature in the body of this article. However for completeness, they are defined as

$$
G_{\mathrm{T}(\overline{\mathrm{T}})}^{i j}(x, y[, \tilde{t}])=\theta\left(x^{0}-y^{0}\right) G_{>(<)}^{i j}(x, y[, \tilde{t}])+\theta\left(y^{0}-x^{0}\right) G_{<(>)}^{i j}(x, y[, \tilde{t}]) .
$$

Self-energies. We follow the sign convention of ref. [72] for the definition of the selfenergies, such that a positive dispersive self-energy correction corresponds to a positive shift in the mass-squared. For example, in the Markovian approximation, we denote the inverse of the momentum-space resummed retarded (advanced) propagator by

$$
D_{R(A)}^{i j}(p) \equiv p^{2} \delta^{i j}-\left[M^{2}\right]^{i j}-\Pi_{R(A)}^{i j}(p),
$$

where we have adopted the notation $D_{R(A)}^{i j}(p)$ from ref. [11]. This inverse appears in ref. [72] as $\Omega_{R(A)}^{i j}(p)$ and in refs. $[11,84]$ as $\Delta_{R(A)}^{-1}(p)$.

The various self-energies satisfy identities analogous to those identified above for the two-point functions. In the case of the analogue of the spectral function,

$$
\Pi_{\rho}^{i j}(x, y[, \tilde{t}])=i \Pi_{>}^{i j}(x, y[, \tilde{t}])-i \Pi_{<}^{i j}(x, y[, \tilde{t}]),
$$

we have introduced the real-valued distribution

$$
\widetilde{\Pi}_{\rho}\left(p, p^{\prime}[, \tilde{t}]\right) \equiv-i \Pi_{\rho}\left(p, p^{\prime}[, \tilde{t}]\right)=-i \mathcal{F}_{y}\left[\mathcal{F}_{x}\left[\Pi_{\rho}(x, y[\tilde{t}])\right](p)\right]\left(-p^{\prime}\right)
$$


CTP Schwinger-Dyson equation. The Schwinger-Dyson equation of the CTP formalism may be derived systematically from the 2PI CJT effective action [104] (see also refs. $[81-83,105])$, which is defined via the Legendre transform $(\hbar=1)$

$$
\Gamma[\phi, G]=-i \ln \mathcal{Z}[J, K]-\int_{\mathcal{C}_{[t]}, x} J(x) \phi(x)-\frac{1}{2} \int_{\mathcal{C}_{[t]}, x, y} K(x, y[\tilde{t}])\left(\phi(x) \phi(y)+G_{\mathcal{C}}(x, y[, \tilde{t}])\right),
$$

where, for simplicity, we consider here the case of single real scalar field $\phi$. The contour integral has the explicit form

$$
\int_{\mathcal{C}_{[t]}, x} \equiv \int \mathrm{d}^{3} \mathbf{x}\left[\int_{-\infty\left[\tilde{t}_{i}\right]+i \epsilon}^{+\infty[\tilde{t}]+i \epsilon} \mathrm{d} x^{0}-\int_{-\infty\left[\tilde{t}_{i}\right]-i \epsilon}^{+\infty[\tilde{t}]-i \epsilon} \mathrm{d} x^{0}\right]
$$

where $\epsilon=0^{+}$and the two terms correspond to the two branches of the CTP contour.

In order to build the generating functional $\mathcal{Z}[J, K]$, we start from the partition function $\mathcal{Z}=\operatorname{tr} \rho$, which is picture- and, in the absence of external sources, time-independent. A path-integral representation of the partition function can be derived by perturbing the system with the introduction of an external test source $J$. Note that the presence of this external source means that the density operator depends explicitly on time in all pictures. Proceeding in the Heisenberg picture, we insert into the partition function complete sets of eigenstates of the Heisenberg picture field operator $\left|\phi(\mathbf{x}), x^{0}\right\rangle$ and construct the path integral in the standard text-book fashion (see e.g. ref. [84]). The density operator gives rise to a term

$$
\left\langle\phi(\mathbf{x}), x^{0}-i \epsilon\left|\rho_{H}(\tilde{t})\right| \phi(\mathbf{x}), x^{0}+i \epsilon\right\rangle_{J},
$$

which we expand in terms of the field eigenvalues and a series of poly-local sources [82, 83]:

$$
\left\langle\phi(\mathbf{x}), x^{0}-i \epsilon\left|\rho_{H}(\tilde{t})\right| \phi(\mathbf{x}), x^{0}+i \epsilon\right\rangle_{J}=\exp (i \mathcal{K}[\phi[, \tilde{t}]])=\exp \left[i \int_{\mathcal{C}_{[\tilde{t}]}, x, y} \phi(x) K(x, y, \tilde{t}) \phi(y)\right]
$$

where, assuming a Gaussian density operator, we have kept only the bi-local source $K(x, y,[, \tilde{t}])$, as appeared in the 2PI effective action (eq. (A.23)). We note that the path integral is a $c$-number and, as such, we are free to interpret it in any picture.

By varying the $2 \mathrm{PI}$ effective action with respect to the resummed CTP propagator $G_{\mathcal{C}}$, we obtain the Schwinger-Dyson equation

$$
G_{\mathcal{C}}^{-1}(x, y[, \tilde{t}])=D_{\mathcal{C}}^{0}(x, y)+K(x, y[\tilde{t}])-\Pi_{\mathcal{C}}(x, y[, \tilde{t}]),
$$

where

$$
D_{\mathcal{C}}^{0}(x, y)=\delta_{\mathcal{C}}^{4}(x, y)\left(-\square_{x}-M^{2}\right)
$$

is the Klein-Gordon operator and $\Pi_{\mathcal{C}}(x, y[, \tilde{t}])$ is the CTP self-energy, whose structure is analogous to that of the CTP propagator. For instance, when $x^{0}\left(y^{0}\right)$ is on the time-ordered branch and $y^{0}\left(x^{0}\right)$ is on the anti-time-ordered branch, $\Pi_{\mathcal{C}}(x, y[, \tilde{t}])$ is equal to $i \Pi_{<(>)}(x, y[, \tilde{t}])$ (with the $i$ conventions detailed above). The contour delta function $\delta_{\mathcal{C}}^{4}(x-y)$ coincides with the usual Dirac delta function if $x^{0}$ and $y^{0}$ lie on the same branch of the CTP contour and is zero otherwise. 
The unique inverse of the Klein-Gordon operator is constrained by the Hermiticity properties and CPT invariance of the action, as well as unitarity and causality (see ref. [84]). In addition, one must provide a boundary condition, which corresponds to the EEV of the normal-ordered product of fields. In the path-integral representation, the latter is encoded in the bi-local source. In this way, the tree-level propagators of the Heisenberg picture encode the initial conditions, whereas those of the interaction picture encode the current state of the system. In each case, the bi-local source $K\left(x, y, \tilde{t}_{i}[\tilde{t}]\right)$ must be proportional to Dirac delta functions that lie respectively on the initial and final time surfaces, i.e.

$$
K\left(x, y, \tilde{t}_{i}[\tilde{t}]\right) \propto \delta\left(x_{0}-\tilde{t}_{i}[\tilde{t}]\right) \delta\left(y_{0}-\tilde{t}_{i}[\tilde{t}]\right) .
$$

It is the differing physical content of the tree-level propagators, which marks the main distinction between the Heisenberg- and interaction-picture realizations of non-equilibrium field theory.

\section{B Discrete symmetry transformations}

In this appendix, we revisit the properties of the theory in eq. (1.1) under the discrete symmetry transformations of parity $P$, time-reversal $T$ and charge-conjugation $C$. In particular, as identified in ref. [72], we emphasize the relevance of these properties to the specification of $C$-symmetric initial conditions both in the Heisenberg- and interactionpicture realizations. The provision of $C$-symmetric initial conditions for the two-point functions (in the case of the Heisenberg picture) or the number densities (in the case of the interaction picture) ensures that any non-zero asymmetry generated in the weak-washout regime arises dynamically and vanishes in the $C$-conserving limit of the theory, as it should. The latter considerations are, of course, irrelevant in the strong-washout regime, since the final asymmetry is independent of the initial conditions.

CPT transformations. In the presence of flavour mixing, it is necessary to consider generalized discrete symmetry transformations, which, in an arbitrary flavour basis, contain additional transformations in flavour space (see e.g. ref. [9]). For the model in eq. (1.1), we have the following transformation properties under the generalized parity $P$, time-reversal $T$ and charge-conjugation $C$ transformations:

a) Parity. Under the linear transformation $P$, the scalar fields transform as [94]

$$
\begin{aligned}
b\left(x^{0}, \mathbf{x}\right)^{P} & =\beta_{P} b\left(x^{0},-\mathbf{x}\right), \\
\bar{b}\left(x^{0}, \mathbf{x}\right)^{P} & =\beta_{P}^{*} \bar{b}\left(x^{0},-\mathbf{x}\right), \\
\psi_{i}\left(x^{0}, \mathbf{x}\right)^{P} & = \pm \psi_{i}\left(x^{0},-\mathbf{x}\right),
\end{aligned}
$$

where the complex phase $\beta_{P}$ satisfies $\left|\beta_{P}\right|^{2}=1$.

b) Time-reversal. Under the anti-linear transformation $T$, the scalar fields transform as [94]

$$
\begin{aligned}
b\left(x^{0}, \mathbf{x}\right)^{T} & =\beta_{T} b\left(-x^{0}, \mathbf{x}\right), \\
\bar{b}\left(x^{0}, \mathbf{x}\right)^{T} & =\beta_{T}^{*} \bar{b}\left(-x^{0}, \mathbf{x}\right), \\
\psi_{i}\left(x^{0}, \mathbf{x}\right)^{T} & =U_{j i} \psi_{i}\left(-x^{0}, \mathbf{x}\right),
\end{aligned}
$$


where the complex phase $\beta_{T}$ satisfies $\left|\beta_{T}\right|^{2}=1$ and $\boldsymbol{U}$ is an orthogonal transformation in flavour space, i.e. $U_{i k} U_{j k}=U_{k i} U_{k j}=\delta_{i j}$.

c) Charge-conjugation. Under the linear transformation $C$, the scalar fields transform as [94]

$$
\begin{aligned}
b(x)^{C} & =\beta_{C} \bar{b}(x), \\
\bar{b}(x)^{C} & =\beta_{C}^{*} b(x), \\
\psi_{i}(x)^{C} & =U_{i j} \psi_{j}(x),
\end{aligned}
$$

where the complex phase $\beta_{C}$ satisfies $\left|\beta_{C}\right|^{2}=1$. In order for the Lagrangian to be invariant under $C P T$, the same orthogonal transformation $\boldsymbol{U}$ must appear in both the generalized $T$ transformation in eq. (B.2c) and the generalized $C$ transformation in eq. (B.3c). This orthogonal transformation $\boldsymbol{U}$ may be either a rotation or a reflection in flavour space, having the general form

$$
U=\left(\begin{array}{cc}
\cos (\alpha) & -\sin (\alpha) \\
\sin (\alpha) & \cos (\alpha)
\end{array}\right) \quad \text { or } \quad U=\left(\begin{array}{cc}
\cos (\alpha) & \sin (\alpha) \\
\sin (\alpha) & -\cos (\alpha)
\end{array}\right),
$$

or an arbitrary product of the rotations and reflections (a product of a rotation and a reflection is still a reflection).

The Lagrangian in eq. (1.1) is invariant under $C$ so long as we can find a phase $\beta_{C}$ and transformation $\boldsymbol{U}$ such that the mass matrix $\boldsymbol{M}^{2}$ and Yukawa couplings $\boldsymbol{h}$ satisfy

$$
\begin{aligned}
U_{m i} M_{m n}^{2} U_{n j} & =M_{i j}^{2}, \\
\beta_{C}^{2} U_{k i} h_{k} & =h_{i}^{*} .
\end{aligned}
$$

In order to analyze the constraint on the Yukawa couplings provided by eq. (B.5b), it is convenient to introduce the dyadic product $H_{i j} \equiv h_{i} h_{j}^{*}$. The second condition eq. (B.5b) may then be recast in the more convenient form

$$
U_{m i} H_{m n} U_{n j}=H_{i j}^{*},
$$

in which the phase $\beta_{C}$ of the complex scalar field has been eliminated.

In the mass eigenbasis, where $\boldsymbol{M}^{2}$ is diagonal, the first condition in eq. (B.5a) can be satisfied for $M_{1}^{2} \neq M_{2}^{2}$ only for rotations and reflections through angles of $\alpha=0$ or $\pi$. If $\boldsymbol{U}$ is a rotation, $C$-invariance follows if $H_{12}=H_{12}^{*}$, i.e. $\operatorname{Im} H_{12}=0$. On the other hand, if $\boldsymbol{U}$ is a reflection, $C$-invariance follows if $H_{12}=-H_{12}^{*}$, i.e. $\operatorname{Re} H_{12}=0$.

Under a general flavour rotation $\boldsymbol{O}$ through an angle $\theta, \operatorname{Im} H_{12}$ is unchanged, since $\boldsymbol{O}$ is orthogonal. Instead, Re $H_{12}$ transforms as

$$
\operatorname{Re} H_{12} \longrightarrow \operatorname{Re} H_{12}^{\prime}=\cos (2 \theta) \operatorname{Re} H_{12}+\frac{1}{2} \sin (2 \theta)\left(H_{11}-H_{22}\right) \text {. }
$$

Therefore, in the degenerate limit $M_{1}^{2}=M_{2}^{2}$, the resulting $O(2)$ invariance of the free theory (see e.g. ref. [9]) means that we may always rotate through an angle

$$
\theta=\frac{1}{2} \arctan \frac{H_{12}+H_{21}}{H_{22}-H_{11}}
$$

to a basis in which $\operatorname{Re} H_{12}=0$. Thus, the Lagrangian is also $C$-conserving in this case. 
The above observations may be conveniently summarized by forming the Jarlskog invariant

$$
J=2 \operatorname{Im} H_{12} \operatorname{Re} H_{12} M_{1} M_{2} \Delta M^{2},
$$

which vanishes when any of the $C$-conserving conditions are satisfied, viz. $\operatorname{Re} H_{12}=0$, $\operatorname{Im} H_{12}=0$ or $M_{1}=M_{2}$. Rotating to a general flavour basis, the Jarlskog invariant may be written in the form

$$
J=\operatorname{Im} \operatorname{Tr} \boldsymbol{H} \boldsymbol{M}^{3} \boldsymbol{H}^{\top} \boldsymbol{M},
$$

providing the familiar basis-independent measure of both $C$ - and $C P$-violation.

As identified above, in the case that $\operatorname{Re} H_{12}=0$, the Lagrangian is invariant under $C$ transformations that include a reflection in flavour space. In the mass eigenbasis, the orthogonal transformation $\boldsymbol{U}$ is necessarily diagonal. The permitted reflections are therefore about angles of 0 and $\pi$, and we see that the two flavours must transform with opposite phases under $C$, i.e.

$$
\psi_{1}(x)^{C}= \pm \psi_{1}(x), \quad \psi_{2}(x)^{C}=\mp \psi_{2}(x),
$$

and, correspondingly, opposite phases under $T$. On the other hand, in the case that $\operatorname{Im} H_{12}=0$, the Lagrangian is invariant under $C$ transformations that include a rotation in flavour space. In the mass eigenbasis, the orthogonal transformation $\boldsymbol{U}$ is now necessarily isotropic. The permitted rotations are about angles of 0 and $\pi$, and we see that the two flavours must transform with equal phases under $C$, i.e.

$$
\psi_{1}(x)^{C}= \pm \psi_{1}(x), \quad \psi_{2}(x)^{C}= \pm \psi_{2}(x),
$$

and, correspondingly, equal phases under T. As identified in ref. [72], the import of this observation is significant for the specification of $C$-symmetric initial conditions.

Heisenberg picture. In order to derive properties of the Wightman propagators under $C$-conjugation, we use their definition in the form

$$
\begin{aligned}
& G_{>}^{i j}(x, y)=\operatorname{Tr}\left[\rho \psi^{i}(x) \psi^{j}(y)\right], \\
& G_{<}^{i j}(x, y)=\operatorname{Tr}\left[\rho \psi^{j}(y) \psi^{i}(x)\right],
\end{aligned}
$$

where $\rho$ is the density matrix. We define charge-conjugated propagators as propagators with only the fields, but not the density matrix, conjugated. This definition corresponds to the intuitive definition that replaces particles with antiparticles. The Wightman propagators then transform under generalized $C$-conjugation as

$$
G_{\gtrless}^{i j}(x, y) \stackrel{C}{\rightarrow} U_{i m} G_{\gtrless}^{m n}(x, y) U_{j n} .
$$

For the case of flavour rotations with $\alpha=0$ or $\pi$, we may readily verify that the propagators are automatically $C$-symmetric. This is consistent with the fact that if $\operatorname{Im} H_{12}=0$ then no asymmetry can be produced irrespective of the value of the propagators at the initial time surface. On the other hand, for the case of flavour reflections with $\alpha=0$ or $\pi$, the propagators are $C$-symmetric only if their off-diagonals vanish at the initial time surface. 
As we demonstrate in section 6 , the requirement of vanishing off-diagonals makes the produced asymmetry proportional to $\operatorname{Re} H_{12}$. In other words, once we impose $C$-symmetric initial conditions on the propagators, the produced asymmetry automatically vanishes if the Lagrangian is $C$-symmetric, as one would expect.

Interaction picture. In the interaction picture, we may begin by fixing the transformation properties of the free field operators under generalized discrete symmetry transformations in Fock space directly [9]. The matrix of number densities is defined by

$$
n^{i j}=\left\langle a_{j}^{\dagger} a_{i}\right\rangle=\operatorname{Tr}\left(\rho a_{j}^{\dagger} a_{i}\right) .
$$

Similarly to the Heisenberg picture, we define $C$-transformation such that it transforms the creation and annihilation operators but not the density matrix.

As follows from eq. (B.12), under rotations $a_{1} \stackrel{C}{\rightarrow} \pm a_{1}$ and $a_{2} \stackrel{C}{\rightarrow} \pm a_{2}$. The additional phase cancels in eq. (B.15) and therefore the matrix of number densities is automatically $C$ symmetric. This is in agreement with the observation that once $\operatorname{Im} H_{12}=0$ no asymmetry can be generated irrespective of the choice of the initial conditions. On the other hand, for reflections $a_{1} \stackrel{C}{\rightarrow} \pm a_{1}$ and $a_{2} \stackrel{C}{\rightarrow} \mp a_{2}$, such that off-diagonals of $n^{i j}$ acquire a relative sign. The condition of $C$-invariance therefore implies that the matrix of number densities must be diagonal at the initial time surface.

\section{Rate equations in the radiation-dominated universe}

Full treatment of leptogenesis would require solving the kinetic equations for all momentum modes. However, such an analysis is technically demanding and has been performed only in a handful of works (see e.g. ref. [106]). Instead, one usually assumes kinetic equilibration of the mixing fields and approximates kinetic equations for the distribution functions by so-called rate equations for the corresponding number densities. In this appendix, we derive the rate equations in the radiation-dominated universe and re-derive the strong-washout approximation formulas presented in ref. [8].

Source and washout terms. Decays $\psi_{i} \rightarrow b b$ as well as inverse decays $\bar{b} \bar{b} \rightarrow \psi_{i}$ increase the asymmetry by two units, wheres decays $\psi_{i} \rightarrow \bar{b} \bar{b}$ and inverse decays $b b \rightarrow \psi_{i}$ decrease the asymmetry by two units. Using the results of appendix E in ref. [58], we obtain, in agreement with this physical picture, the time-derivative of the asymmetry

$$
\begin{aligned}
\frac{\mathrm{d} \eta}{\mathrm{d} t}= & \int \mathrm{d} \Pi_{\mathbf{q}}^{3} \mathrm{~d} \Pi_{\mathbf{p}}^{3} \mathrm{~d} \Pi_{\mathbf{k}}^{3}(2 \pi)^{4} \delta^{4}(k-p-q) \\
& \times\left\{H_{i j}\left(\left[1+n^{i j}(k)\right] n_{\bar{b}}(p) n_{\bar{b}}(q)-n^{i j}(k)\left[1+n_{\bar{b}}(p)\right]\left[1+n_{\bar{b}}(q)\right]\right)\right. \\
& \left.\quad-H_{i j}^{*}\left(\left[1+n^{i j}(k)\right] n_{b}(p) n_{b}(q)-n^{i j}(k)\left[1+n_{b}(p)\right]\left[1+n_{b}(q)\right]\right)\right\},
\end{aligned}
$$

where $\mathrm{d} \Pi_{\mathbf{q}}^{3} \equiv \frac{\mathrm{d}^{3} \mathbf{q}}{(2 \pi)^{3}} \frac{1}{2 E}$ is the Lorentz-invariant phase-space element. One can easily recognize the usual structure of the gain and loss terms in eq. (C.1).

It is common to approximate the r.h.s. of eq. (C.1) by the difference of the source and washout terms. The source term is defined as the r.h.s. with distribution functions of the 
complex field set to equilibrium, $n_{b}=n_{\bar{b}}=n_{\text {eq }}$. By detailed balance, the contribution of the equilibrium part of $n^{i j}$ vanishes, and we are left with the source term

$$
S=4 \operatorname{Im} H_{12} \int \mathrm{d} \Pi_{\mathbf{k}}^{3} \widetilde{\Pi}_{\rho}(k) \operatorname{Im} \delta n^{12}(k),
$$

where the function $\widetilde{\Pi}_{\rho}$ is defined in eq. (2.26). The assumption of kinetic equilibrium amounts to

$$
\delta n^{12}(k) \approx \frac{\delta N^{12}}{N_{\mathrm{eq}}} n_{\mathrm{eq}}(k),
$$

where $N$ denotes the total particle number density. It is furthermore common to approximate $n_{\mathrm{eq}}$ by the Boltzmann distribution. Substituting eq. (C.3) into eq. (2.2) and approximating $\widetilde{\Pi}_{\rho}$ by its low-temperature limit, we then find

$$
S=\frac{\operatorname{Im} H_{12}}{4 \pi \bar{M}} \frac{K_{1}(\bar{M} / T)}{K_{2}(\bar{M} / T)} \operatorname{Im} \delta N^{12},
$$

where $K_{1}$ and $K_{2}$ are modified Bessel functions of the second kind.

The washout term is defined by setting the density matrix of the mixing fields to its equilibrium form, $\boldsymbol{n}=\mathbf{1} \cdot n_{\mathrm{eq}}$. After some straightforward algebra we find

$$
\begin{aligned}
W= & \sum_{i} H_{i i} \int \mathrm{d} \Pi_{\mathbf{q}}^{3} \mathrm{~d} \Pi_{\mathbf{p}}^{3} \mathrm{~d} \Pi_{\mathbf{k}}^{3}(2 \pi)^{4} \delta^{4}(k-p-q) \\
& \times\left[n_{b}(p)-n_{\bar{b}}(p)\right]\left[n_{b}(p)+n_{\bar{b}}(p)-n_{\mathrm{eq}}(k)\right] .
\end{aligned}
$$

Here, we again use Boltzmann statistics for the distribution functions, $n_{b} \approx \exp (-(E-$ $\mu) / T)$ and $n_{\bar{b}} \approx \exp (-(E+\mu) / T)$, where $\mu$ is the chemical potential. Neglecting further the (quantum-statistical) term $n_{\text {eq }}(k)$ and expanding to the first order in $\mu$, we obtain

$$
W \approx-2 \eta \bar{\Gamma} \frac{\bar{M}^{2}}{T^{2}} K_{1}(\bar{M} / T) .
$$

Generalization to radiation-dominated universe. In the expanding universe, one can recast the kinetic equation for the asymmetry in the form similar to eq. (C.1) by using the co-moving number densities [107]:

$$
\frac{1}{a} \frac{\mathrm{d} \eta}{\mathrm{d} t}=\frac{\operatorname{Im} H_{12}}{4 \pi \bar{M}} \frac{K_{1}(\bar{M} / T)}{K_{2}(\bar{M} / T)} \operatorname{Im} \delta N^{12}-2 \eta \bar{\Gamma} \frac{\bar{M}^{2}}{T^{2}} K_{1}(\bar{M} / T),
$$

where $t$ is now the conformal time, $a$ is the scale factor, and $\eta$ and $\delta N^{12}$ are the comoving number densities. In the radiation-dominated universe $a(t)=a_{R} t$, where $a_{R}$ is constant. Following ref. [7], we choose $a_{R}=M_{\mathrm{Pl}}\left(45 / 4 \pi^{3} g_{*}\right)^{\frac{1}{2}}$ with $M_{\mathrm{Pl}}$ being the Planck mass, and $g_{*}$ the effective number of massless degrees of freedom. For this choice, $z \equiv \bar{M} / T=\bar{M} t$. The resulting rate equation for the asymmetry reads

$$
\frac{\mathrm{d} \eta}{\mathrm{d} z}=\frac{a_{R} z}{\bar{M}^{2}}\left[\frac{\operatorname{Im} H_{12}}{4 \pi \bar{M}} \frac{K_{1}(z)}{K_{2}(z)} \operatorname{Im} \delta N^{12}-2 \eta \bar{\Gamma} z^{2} K_{1}(z)\right] .
$$


Strong-washout approximation. It follows from eq. (C.8) that the larger the washout parameter

$$
\kappa \equiv \frac{a_{R} \bar{\Gamma}}{\bar{M}^{2}},
$$

the more asymmetry is washed out by the inverse decay processes. If $\kappa \gg 1$, then one speaks of the strong-washout regime. In the strong-washout regime, the final asymmetry does not depend on the initial conditions and most of the asymmetry is produced after the temperature drops below the mass of the heavy decaying particle (see e.g. ref. [108]). For $T<\bar{M}$, the density matrix $\boldsymbol{n}$ is suppressed at momenta $|\mathbf{q}|>\bar{M}$, such that, in eq. (4.4), we can approximate $\bar{\omega}$ by $\bar{M}$. Integrating eq. (4.4) over the phase space and switching to the co-moving number densities, we obtain in this approximation

$$
\frac{\mathrm{d} \delta \boldsymbol{N}}{\mathrm{d} z}+\mathbf{1} \frac{\mathrm{d} N_{\mathrm{eq}}}{\mathrm{d} z}+i \frac{a_{R} z}{\bar{M}^{3}}\left[\boldsymbol{M}^{2}, \delta \boldsymbol{N}\right]=\frac{a_{R} z}{\bar{M}^{3}}\left\{\widetilde{\boldsymbol{\Pi}}_{\rho}, \delta \boldsymbol{N}\right\}
$$

(see refs. [7, 8] for the details of the derivation). In ref. [74], it was proposed that, in a strong-washout regime, one can obtain an approximate solution for $\delta \boldsymbol{N}$ at late times by neglecting the derivative of $\delta \boldsymbol{N}$. This solution reads

$$
\delta N^{12} \approx \frac{\operatorname{Re} H_{12} \operatorname{Tr} \boldsymbol{H}\left(2 \bar{M} \bar{\Gamma}-i \Delta M_{12}^{2}\right) \bar{M}^{2}}{H_{11} H_{22}\left(\Delta M_{12}^{2}\right)^{2}+16 \bar{M}^{2} \bar{\Gamma}^{2} \operatorname{det} \operatorname{Re} \boldsymbol{H}} \frac{\bar{M}}{a_{R} z} \frac{\mathrm{d} N_{\mathrm{eq}}}{\mathrm{d} z} \approx \frac{-i}{8 \pi} \frac{\operatorname{Re} H_{12}}{\Delta M_{12}^{2}} \frac{\bar{\Gamma}^{2}}{\Gamma_{1} \Gamma_{2}} \frac{1}{\kappa z} \frac{\mathrm{d} N_{\mathrm{eq}}}{\mathrm{d} z},
$$

where the second approximate equality is valid for $\left|\Delta M_{12}^{2}\right| \gg \bar{M} \bar{\Gamma}$. It is interesting to note that the solution is proportional to Re $H_{12}$. This implies that the resulting source term automatically vanishes if either of the $C$-symmetry conditions, $\operatorname{Im} H_{12}=0$ or $\operatorname{Re} H_{12}=0$, is fulfilled.

Open Access. This article is distributed under the terms of the Creative Commons Attribution License (CC-BY 4.0), which permits any use, distribution and reproduction in any medium, provided the original author(s) and source are credited.

\section{References}

[1] Particle Data Group collaboration, K.A. Olive et al., Review of particle physics, Chin. Phys. C 38 (2014) 090001 [inSPIRE].

[2] A. Pais and O. Piccioni, Note on the decay and absorption of the $\theta^{0}$, Phys. Rev. 100 (1955) 1487 [INSPIRE].

[3] D. Boyanovsky and C.M. Ho, Nonequilibrium dynamics of mixing, oscillations and equilibration: a model study, Phys. Rev. D 75 (2007) 085004 [hep-ph/0610036] [INSPIRE].

[4] D. Boyanovsky and L. Lello, Time evolution of cascade decay, New J. Phys. 16 (2014) 063050 [arXiv: 1403.6366] [inSPIRE].

[5] D. Boyanovsky, Space-time evolution of heavy sterile neutrinos in cascade decays, Nucl. Phys. B 888 (2014) 248 [arXiv:1406.5739] [INSPIRE].

[6] D. Boyanovsky, Nearly degenerate heavy sterile neutrinos in cascade decay: mixing and oscillations, Phys. Rev. D 90 (2014) 105024 [arXiv:1409.4265] [INSPIRE]. 
[7] B. Garbrecht and M. Herranen, Effective theory of resonant leptogenesis in the closed-time-path approach, Nucl. Phys. B 861 (2012) 17 [arXiv:1112.5954] [INSPIRE].

[8] B. Garbrecht, F. Gautier and J. Klaric, Strong washout approximation to resonant leptogenesis, JCAP 09 (2014) 033 [arXiv: 1406.4190] [INSPIRE].

[9] P.S. Bhupal Dev, P. Millington, A. Pilaftsis and D. Teresi, Flavour covariant transport equations: an application to resonant leptogenesis, Nucl. Phys. B 886 (2014) 569 [arXiv: 1404.1003] [INSPIRE].

[10] P.S. Bhupal Dev, P. Millington, A. Pilaftsis and D. Teresi, Corrigendum to "Flavour covariant transport equations: an application to resonant leptogenesis", Nucl. Phys. B 897 (2015) 749 [arXiv:1504.07640] [InSPIRE].

[11] P.S. Bhupal Dev, P. Millington, A. Pilaftsis and D. Teresi, Kadanoff-Baym approach to flavour mixing and oscillations in resonant leptogenesis, Nucl. Phys. B 891 (2015) 128 [arXiv: 1410.6434] [INSPIRE].

[12] M. Fukugita and T. Yanagida, Baryogenesis without grand unification, Phys. Lett. B 174 (1986) 45 [inSPIRE].

[13] S. Blanchet and P. Di Bari, The minimal scenario of leptogenesis, New J. Phys. 14 (2012) 125012 [arXiv:1211.0512] [INSPIRE].

[14] V.A. Kuzmin, V.A. Rubakov and M.E. Shaposhnikov, On the anomalous electroweak baryon number nonconservation in the early universe, Phys. Lett. B 155 (1985) 36 [INSPIRE].

[15] A. Pilaftsis, CP violation and baryogenesis due to heavy Majorana neutrinos, Phys. Rev. D 56 (1997) 5431 [hep-ph/9707235] [INSPIRE].

[16] A. Pilaftsis, Heavy Majorana neutrinos and baryogenesis, Int. J. Mod. Phys. A 14 (1999) 1811 [hep-ph/9812256] [InSPIRE].

[17] J.R. Ellis, M. Raidal and T. Yanagida, Observable consequences of partially degenerate leptogenesis, Phys. Lett. B 546 (2002) 228 [hep-ph/0206300] [INSPIRE].

[18] T. Endoh, T. Morozumi and Z. Xiong, Primordial lepton family asymmetries in seesaw model, Prog. Theor. Phys. 111 (2004) 123 [hep-ph/0308276] [InSPIRE].

[19] A. Pilaftsis, Resonant $\tau$ leptogenesis with observable lepton number violation, Phys. Rev. Lett. 95 (2005) 081602 [hep-ph/0408103] [INSPIRE].

[20] A. Pilaftsis and T.E.J. Underwood, Electroweak-scale resonant leptogenesis, Phys. Rev. D 72 (2005) 113001 [hep-ph/0506107] [INSPIRE].

[21] T. Asaka and M. Shaposhnikov, The $\nu M S M$, dark matter and baryon asymmetry of the universe, Phys. Lett. B 620 (2005) 17 [hep-ph/0505013] [INSPIRE].

[22] O. Vives, Flavor dependence of CP asymmetries and thermal leptogenesis with strong right-handed neutrino mass hierarchy, Phys. Rev. D 73 (2006) 073006 [hep-ph/0512160] [INSPIRE].

[23] F.F. Deppisch and A. Pilaftsis, Lepton flavour violation and $\theta_{13}$ in minimal resonant leptogenesis, Phys. Rev. D 83 (2011) 076007 [arXiv: 1012.1834] [INSPIRE].

[24] S. Blanchet, P. Di Bari, D.A. Jones and L. Marzola, Leptogenesis with heavy neutrino flavours: from density matrix to Boltzmann equations, JCAP 01 (2013) 041 [arXiv:1112.4528] [INSPIRE]. 
[25] R. Barbieri, P. Creminelli, A. Strumia and N. Tetradis, Baryogenesis through leptogenesis, Nucl. Phys. B 575 (2000) 61 [hep-ph/9911315] [INSPIRE].

[26] A. Abada, S. Davidson, F.-X. Josse-Michaux, M. Losada and A. Riotto, Flavour issues in leptogenesis, JCAP 04 (2006) 004 [hep-ph/0601083] [INSPIRE].

[27] E. Nardi, Y. Nir, E. Roulet and J. Racker, The importance of flavor in leptogenesis, JHEP 01 (2006) 164 [hep-ph/0601084] [inSPIRE].

[28] A. Abada et al., Flavour matters in leptogenesis, JHEP 09 (2006) 010 [hep-ph/0605281] [INSPIRE].

[29] S. Blanchet and P. Di Bari, Flavour effects on leptogenesis predictions, JCAP 03 (2007) 018 [hep-ph/0607330] [INSPIRE].

[30] S. Blanchet, P. Di Bari and G.G. Raffelt, Quantum Zeno effect and the impact of flavour in leptogenesis, JCAP 03 (2007) 012 [hep-ph/0611337] [INSPIRE].

[31] S. Pascoli, S.T. Petcov and A. Riotto, Leptogenesis and low energy CP-violation in neutrino physics, Nucl. Phys. B 774 (2007) 1 [hep-ph/0611338] [INSPIRE].

[32] G.C. Branco, A.J. Buras, S. Jäger, S. Uhlig and A. Weiler, Another look at minimal lepton flavour violation, leptogenesis and the ratio $M_{\nu} / \Lambda_{\mathrm{LFV}}$, JHEP 09 (2007) 004 [hep-ph/0609067] [INSPIRE].

[33] A. De Simone and A. Riotto, On the impact of flavour oscillations in leptogenesis, JCAP 02 (2007) 005 [hep-ph/0611357] [INSPIRE].

[34] A. Pilaftsis, Resonant CP-violation induced by particle mixing in transition amplitudes, Nucl. Phys. B 504 (1997) 61 [hep-ph/9702393] [INSPIRE].

[35] A. Pilaftsis and T.E.J. Underwood, Resonant leptogenesis, Nucl. Phys. B 692 (2004) 303 [hep-ph/0309342] [INSPIRE].

[36] M. Flanz, E.A. Paschos and U. Sarkar, Baryogenesis from a lepton asymmetric universe, Phys. Lett. B 345 (1995) 248 [Erratum ibid. B 382 (1996) 447] [hep-ph/9411366] [INSPIRE].

[37] L. Covi and E. Roulet, Baryogenesis from mixed particle decays, Phys. Lett. B 399 (1997) 113 [hep-ph/9611425] [INSPIRE].

[38] L. Covi, E. Roulet and F. Vissani, CP violating decays in leptogenesis scenarios, Phys. Lett. B 384 (1996) 169 [hep-ph/9605319] [INSPIRE].

[39] W. Buchmüller and M. Plümacher, CP asymmetry in Majorana neutrino decays, Phys. Lett. B 431 (1998) 354 [hep-ph/9710460] [INSPIRE].

[40] P.S. Bhupal Dev, P. Millington, A. Pilaftsis and D. Teresi, Flavour effects in resonant leptogenesis from semi-classical and Kadanoff-Baym approaches, J. Phys. Conf. Ser. 631 (2015) 012087 [arXiv:1502.07987] [INSPIRE].

[41] E.W. Kolb and S. Wolfram, Baryon number generation in the early universe, Nucl. Phys. B 172 (1980) 224 [Erratum ibid. B 195 (1982) 542] [INSPIRE].

[42] M.A. Luty, Baryogenesis via leptogenesis, Phys. Rev. D 45 (1992) 455 [InSPIRE].

[43] G. Sigl and G. Raffelt, General kinetic description of relativistic mixed neutrinos, Nucl. Phys. B 406 (1993) 423 [INSPIRE]. 
[44] E.K. Akhmedov, V.A. Rubakov and A.Y. Smirnov, Baryogenesis via neutrino oscillations, Phys. Rev. Lett. 81 (1998) 1359 [hep-ph/9803255] [INSPIRE].

[45] M. Shaposhnikov, The $\nu M S M$, leptonic asymmetries and properties of singlet fermions, JHEP 08 (2008) 008 [arXiv:0804.4542] [INSPIRE].

[46] J.-S. Gagnon and M. Shaposhnikov, Baryon asymmetry of the universe without Boltzmann or Kadanoff-Baym equations, Phys. Rev. D 83 (2011) 065021 [arXiv:1012.1126] [INSPIRE].

[47] T. Asaka, S. Eijima and H. Ishida, Kinetic equations for baryogenesis via sterile neutrino oscillation, JCAP 02 (2012) 021 [arXiv:1112.5565] [INSPIRE].

[48] L. Canetti, M. Drewes, T. Frossard and M. Shaposhnikov, Dark matter, baryogenesis and neutrino oscillations from right-handed neutrinos, Phys. Rev. D 87 (2013) 093006 [arXiv: 1208.4607] [INSPIRE].

[49] B. Shuve and I. Yavin, Baryogenesis through neutrino oscillations: a unified perspective, Phys. Rev. D 89 (2014) 075014 [arXiv:1401.2459] [INSPIRE].

[50] W. Buchmüller and S. Fredenhagen, Quantum mechanics of baryogenesis, Phys. Lett. B 483 (2000) 217 [hep-ph/0004145] [INSPIRE].

[51] T. Prokopec, M.G. Schmidt and S. Weinstock, Transport equations for chiral fermions to order $\hbar$ and electroweak baryogenesis: part I, Annals Phys. 314 (2004) 208 [hep-ph/0312110] [INSPIRE].

[52] T. Prokopec, M.G. Schmidt and S. Weinstock, Transport equations for chiral fermions to order $\hbar$ and electroweak baryogenesis: part II, Annals Phys. 314 (2004) 267 [hep-ph/0406140] [INSPIRE].

[53] A. De Simone and A. Riotto, Quantum Boltzmann equations and leptogenesis, JCAP 08 (2007) 002 [hep-ph/0703175] [INSPIRE].

[54] A. De Simone and A. Riotto, On resonant leptogenesis, JCAP 08 (2007) 013 [arXiv: 0705.2183] [INSPIRE].

[55] V. Cirigliano, A. De Simone, G. Isidori, I. Masina and A. Riotto, Quantum resonant leptogenesis and minimal lepton flavour violation, JCAP 01 (2008) 004 [arXiv:0711.0778] [INSPIRE].

[56] A. Anisimov, W. Buchmüller, M. Drewes and S. Mendizabal, Nonequilibrium dynamics of scalar fields in a thermal bath, Annals Phys. 324 (2009) 1234 [arXiv:0812.1934] [INSPIRE].

[57] M. Garny, A. Hohenegger, A. Kartavtsev and M. Lindner, Systematic approach to leptogenesis in nonequilibrium QFT: vertex contribution to the CP-violating parameter, Phys. Rev. D 80 (2009) 125027 [arXiv:0909.1559] [INSPIRE].

[58] M. Garny, A. Hohenegger, A. Kartavtsev and M. Lindner, Systematic approach to leptogenesis in nonequilibrium QFT: self-energy contribution to the CP-violating parameter, Phys. Rev. D 81 (2010) 085027 [arXiv:0911.4122] [INSPIRE].

[59] V. Cirigliano, C. Lee, M.J. Ramsey-Musolf and S. Tulin, Flavored quantum Boltzmann equations, Phys. Rev. D 81 (2010) 103503 [arXiv:0912.3523] [InSPIRE].

[60] A. Anisimov, W. Buchmüller, M. Drewes and S. Mendizabal, Leptogenesis from quantum interference in a thermal bath, Phys. Rev. Lett. 104 (2010) 121102 [arXiv:1001.3856] [INSPIRE]. 
[61] M. Garny, A. Hohenegger and A. Kartavtsev, Medium corrections to the CP-violating parameter in leptogenesis, Phys. Rev. D 81 (2010) 085028 [arXiv:1002.0331] [INSPIRE].

[62] M. Beneke, B. Garbrecht, M. Herranen and P. Schwaller, Finite number density corrections to leptogenesis, Nucl. Phys. B 838 (2010) 1 [arXiv: 1002.1326] [InSPIRE].

[63] M. Beneke, B. Garbrecht, C. Fidler, M. Herranen and P. Schwaller, Flavoured leptogenesis in the CTP formalism, Nucl. Phys. B 843 (2011) 177 [arXiv:1007.4783] [InSPIRE].

[64] B. Garbrecht, Leptogenesis: the other cuts, Nucl. Phys. B 847 (2011) 350 [arXiv: 1011.3122] [INSPIRE].

[65] A. Anisimov, W. Buchmüller, M. Drewes and S. Mendizabal, Quantum leptogenesis I, Annals Phys. 326 (2011) 1998 [Erratum ibid. 338 (2011) 376] [arXiv:1012.5821] [InSPIRE].

[66] M. Garny, A. Kartavtsev and A. Hohenegger, Leptogenesis from first principles in the resonant regime, Annals Phys. 328 (2013) 26 [arXiv:1112.6428] [INSPIRE].

[67] M. Drewes and B. Garbrecht, Leptogenesis from a GeV seesaw without mass degeneracy, JHEP 03 (2013) 096 [arXiv:1206.5537] [INSPIRE].

[68] B. Garbrecht, Baryogenesis from mixing of lepton doublets, Nucl. Phys. B 868 (2013) 557 [arXiv: 1210.0553 ] [INSPIRE].

[69] T. Frossard, M. Garny, A. Hohenegger, A. Kartavtsev and D. Mitrouskas, Systematic approach to thermal leptogenesis, Phys. Rev. D 87 (2013) 085009 [arXiv:1211.2140] [INSPIRE].

[70] M. Drewes, The phenomenology of right handed neutrinos, Int. J. Mod. Phys. E 22 (2013) 1330019 [arXiv:1303.6912] [InSPIRE].

[71] B. Garbrecht and M.J. Ramsey-Musolf, Cuts, cancellations and the closed time path: the soft leptogenesis example, Nucl. Phys. B 882 (2014) 145 [arXiv:1307.0524] [INSPIRE].

[72] A. Hohenegger and A. Kartavtsev, Leptogenesis in crossing and runaway regimes, JHEP 07 (2014) 130 [arXiv: 1404.5309] [INSPIRE].

[73] S. Iso, K. Shimada and M. Yamanaka, Kadanoff-Baym approach to the thermal resonant leptogenesis, JHEP 04 (2014) 062 [arXiv:1312.7680] [INSPIRE].

[74] S. Iso and K. Shimada, Coherent flavour oscillation and CP-violating parameter in thermal resonant leptogenesis, JHEP 08 (2014) 043 [arXiv: 1404.4816] [INSPIRE].

[75] G. Baym and L.P. Kadanoff, Conservation laws and correlation functions, Phys. Rev. 124 (1961) 287 [INSPIRE].

[76] L.P. Kadanoff and G. Baym, Quantum statistical mechanics, Benjamin, New York U.S.A. (1962).

[77] J.-P. Blaizot and E. Iancu, The quark gluon plasma: collective dynamics and hard thermal loops, Phys. Rept. 359 (2002) 355 [hep-ph/0101103] [INSPIRE].

[78] J. Berges, Introduction to nonequilibrium quantum field theory, AIP Conf. Proc. 739 (2004) 3 [hep-ph/0409233] [InSPIRE].

[79] J.S. Schwinger, Brownian motion of a quantum oscillator, J. Math. Phys. 2 (1961) 407 [INSPIRE].

[80] L.V. Keldysh, Diagram technique for nonequilibrium processes, Zh. Eksp. Teor. Fiz. 47 (1964) 1515 [INSPIRE]. 
[81] R.D. Jordan, Effective field equations for expectation values, Phys. Rev. D 33 (1986) 444 [INSPIRE].

[82] E. Calzetta and B.L. Hu, Nonequilibrium quantum fields: closed time path effective action, Wigner function and Boltzmann equation, Phys. Rev. D 37 (1988) 2878 [INSPIRE].

[83] E. Calzetta and B.L. Hu, Closed time path functional formalism in curved space-time: application to cosmological back reaction problems, Phys. Rev. D 35 (1987) 495 [INSPIRE].

[84] P. Millington and A. Pilaftsis, Perturbative nonequilibrium thermal field theory, Phys. Rev. D 88 (2013) 085009 [arXiv:1211.3152] [inSPIRE].

[85] P. Millington and A. Pilaftsis, Perturbative non-equilibrium thermal field theory to all orders in gradient expansion, Phys. Lett. B 724 (2013) 56 [arXiv:1304.7249] [INSPIRE].

[86] H.A. Weldon, Thermalization of boson propagators in finite-temperature field theory, Phys. Rev. D 45 (1992) 352 [InSPIRE].

[87] T. Altherr and D. Seibert, Problems of perturbation series in nonequilibrium quantum field theories, Phys. Lett. B 333 (1994) 149 [hep-ph/9405396] [INSPIRE].

[88] T. Altherr, Resummation of perturbation series in nonequilibrium scalar field theory, Phys. Lett. B 341 (1995) 325 [hep-ph/9407249] [INSPIRE].

[89] P.F. Bedaque, Thermalization and pinch singularities in non-equilibrium quantum field theory, Phys. Lett. B 344 (1995) 23 [hep-ph/9410415] [INSPIRE].

[90] I. Dadić, Two mechanisms for elimination of pinch singularities in out of equilibrium thermal field theories, Phys. Rev. D 59 (1999) 125012 [hep-ph/9801399] [INSPIRE].

[91] C. Greiner and S. Leupold, Interpretation and resolution of pinch singularities in non-equilibrium quantum field theory, Eur. Phys. J. C 8 (1999) 517 [hep-ph/9804239] [INSPIRE].

[92] B. Garbrecht and M. Garny, Finite width in out-of-equilibrium propagators and kinetic theory, Annals Phys. 327 (2012) 914 [arXiv:1108.3688] [INSPIRE].

[93] M. Garny, A. Hohenegger and A. Kartavtsev, Quantum corrections to leptogenesis from the gradient expansion, arXiv: 1005.5385 [INSPIRE].

[94] A. Hohenegger and A. Kartavtsev, Basis invariant measure of CP-violation and renormalization, Nucl. Phys. B 899 (2015) 476 [arXiv:1309.1385] [INSPIRE].

[95] A.D. Sakharov, Violation of CP invariance, $C$ asymmetry and baryon asymmetry of the universe, Pisma Zh. Eksp. Teor. Fiz. 5 (1967) 32 [INSPIRE].

[96] C. Fidler, M. Herranen, K. Kainulainen and P.M. Rahkila, Flavoured quantum Boltzmann equations from cQPA, JHEP 02 (2012) 065 [arXiv: 1108.2309] [INSPIRE].

[97] R.F. Hoskins and J.S. Pinto, Theories of generalised functions: distributions, ultradistributions and other generalised functions, Woodhead Publishing, Cambridge U.K. (2005), pp. 48-50.

[98] N. Nakanishi, A theory of clothed unstable particles, Prog. Theor. Phys. 19 (1958) 607.

[99] T. Petrosky, I. Prigogine and S. Tasaki, Quantum theory of non-integrable systems, Physica A 173 (1991) 175.

[100] M. Le Bellac, Thermal field theory, Cambridge University Press, Cambridge U.K. (2000). 
[101] M.J.G. Veltman, Unitarity and causality in a renormalizable field theory with unstable particles, Physica 29 (1963) 186 [INSPIRE].

[102] M. Plümacher, Baryon asymmetry, neutrino mixing and supersymmetric $\mathrm{SO}(10)$ unification, Nucl. Phys. B 530 (1998) 207 [hep-ph/9704231] [InSPIRE].

[103] A. Hohenegger, A. Kartavtsev and M. Lindner, Deriving Boltzmann equations from Kadanoff-Baym equations in curved space-time, Phys. Rev. D 78 (2008) 085027 [arXiv:0807.4551] [INSPIRE].

[104] J.M. Cornwall, R. Jackiw and E. Tomboulis, Effective action for composite operators, Phys. Rev. D 10 (1974) 2428 [inSPIRE].

[105] R.E. Norton and J.M. Cornwall, On the formalism of relativistic many body theory, Annals Phys. 91 (1975) 106 [INSPIRE].

[106] F. Hahn-Woernle, M. Plümacher and Y.Y.Y. Wong, Full Boltzmann equations for leptogenesis including scattering, JCAP 08 (2009) 028 [arXiv:0907.0205] [INSPIRE].

[107] A. Kartavtsev and D. Besak, Baryogenesis via leptogenesis in an inhomogeneous universe, Phys. Rev. D 78 (2008) 083001 [arXiv:0803.2729] [InSPIRE].

[108] W. Buchmüller, P. Di Bari and M. Plümacher, Leptogenesis for pedestrians, Annals Phys. 315 (2005) 305 [hep-ph/0401240] [INSPIRE]. 